\title{
Synthesis and Biological Activity of Some New Pyrazoline and Pyrimidine Derivatives
}

\author{
Seham Y. Hassan \\ Department of Chemistry, Faculty of Science, University of Alexandria, \\ PO Box 426, 21321 Ibrahimia, Alexandria, Egypt
}

\begin{abstract}
Novas séries de pirazolinas, 3-aril-4,5-diidro-1H-pirazol-1-carbaldeídos (4-6), (aril-4,5-diidro$1 H$-pirazol-1-il)etanonas (9-11) e 3-aril-4,5-diidro-1H-pirazóis (24 e 25) foram sintetizadas pela reação de chalconas (1-3) com hidrato de hidrazina em ácido fórmico, ácido acético ou etanol, respectivamente. Novos derivados de pirimidina 6-arilpirimidina-2-amina (32-34) também foram sintetizados a partir das mesmas chalconas de partida. As estruturas dos novos compostos sintetizados foram estabelecidas através do estudo dos espectros de IV, ${ }^{1} \mathrm{H}$ RMN,${ }^{13} \mathrm{C}$ RMN e análise elementar. Todos os compostos foram avaliados quanto as suas atividades antibacteriana e antifúngica. Dentre estes compostos, três mostraram atividade relevante contra $C$. albicans e outros também apresentaram atividade contra E. coli.
\end{abstract}

New series of pyrazoline 3-aryl-4,5-dihydro-1H-pyrazole-1-carbaldehydes (4-6), (aryl-4,5dihydro-1 $H$-pyrazol-1-yl)ethanones (9-11) and 3-aryl-4,5-dihydro- $1 H$-pyrazoles (24 and 25) were synthesized by reacting chalcones (1-3) with hydrazine hydrate in either formic acid, acetic acid or ethanol, respectively. Also, new 6-arylpyrimidin-2-amine derivatives (32-34) were synthesized from the same chalcones. The structures of the newly synthesized compounds were established on the basis of IR, ${ }^{1} \mathrm{H}$ NMR, ${ }^{13} \mathrm{C}$ NMR, mass spectral data and elemental analyses. The compounds were evaluated for their antibacterial and antifungal activities. Three heterocycles showed relevant activity against $C$. albicans and some compounds also showed activity against $E$. coli.

Keywords: chalcones, pyrazoline, pyrimidine, antibacterial, antifungal

\section{Introduction}

Chalcones are well known intermediates for the synthesis of various heterocyclic compounds. Compounds with the chalcone backbone have been informed to possess various biological activities. ${ }^{1}$ Chalcones have been reported to possess antimicrobial, ${ }^{2}$ anti-inflammatory, ${ }^{2,3}$ antioxidant and anticancer properties. ${ }^{4}$ They were also found to exhibit analgesic, ${ }^{5}$ platelet antiaggregation, ${ }^{6}$ antiulcerative, ${ }^{7}$ antimalarial, ${ }^{8}$ antiviral, ${ }^{9}$ antileishmanial, ${ }^{10}$ antitubercular ${ }^{11}$ and antihyperglycemic properties, ${ }^{12}$ as well as to inhibit the enzymes tyrosinase,${ }^{13}$ and aldose reductase. ${ }^{9}$

Much attention has been paid to the synthesis of heterocyclic compounds bearing nitrogen-containing rings, like pyrazoline and pyrimidine systems, mainly due to their potential pharmacological activity. ${ }^{14-31}$

Pyrazolines are well known and important nitrogencontaining 5-membered heterocycles, which were found to possess a broad spectrum of biological activities such as anti-inflammatory, ${ }^{14}$ herbicidal, ${ }^{15}$ antimicrobial, ${ }^{16}$ antifungal, ${ }^{17}$ antidepressant, ${ }^{18}$ anticonvulsant, ${ }^{19}$ antitumor, ${ }^{20}$ antitubercular, ${ }^{21}$ insecticidal, ${ }^{22}$ antimycobacterial, ${ }^{23}$ molluscicidal, ${ }^{24}$ and antinociceptive. ${ }^{25} \mathrm{~A}$ classical synthesis of 2-pyrazolines involves the base catalyzed ${ }^{26}$ ClaisenSchmidt condensation of appropriate ketones with suitable aldehydes in the presence of potassium hydroxide in aqueous ethanolic solution at room temperature to give chalcones, ${ }^{27}$ which undergo a subsequent cyclization reaction with hydrazines. ${ }^{28}$ Several alternatives are available for this condensation, ${ }^{29}$ including under acidic ${ }^{28}$ or basic $^{30}$ conditions. On the other hand, pyrimidines have also been reported to show a variety of biological activities. ${ }^{31}$

Based on the interest in the above biological activities exhibited by the pyrazoline and pyrimidine compounds, we report here the synthesis of a new series of pyrazoline and pyrimidine compounds.

*e-mail: sehamyassen@yahoo.com 


\section{Results and Discussion}

\section{Chemistry}

The sequence leading to the formation of the title compounds is outlined in Schemes 1 and 2. The desired chalcones (1-3) were prepared by the reaction of anthracene-9-carbaldehyde with different ketones ( $p$-chloro acetophenone, $p$-methyl acetophenone or 2-acetylfuran) in the presence of aqueous ethanolic $\mathrm{KOH}$. The IR spectra of 1-3 exhibited a band due to the unsaturated carbonyl group at $1651-1660 \mathrm{~cm}^{-1}$. Their ${ }^{1} \mathrm{H}$ NMR spectra showed a signal at $\delta 7.45-7.83 \mathrm{ppm}$ attributed to the $=\mathrm{CH}-2$ proton adjacent to $\mathrm{C}=\mathrm{O}$ with a coupling constant $16 \mathrm{~Hz}$, and a doublet at the range $\delta 8.60-8.79 \mathrm{ppm}$ due to the $=\mathrm{CH}-3$ proton with the same coupling constant, which confirmed the presence of chalcones in the trans form. The ${ }^{13} \mathrm{C}$ NMR of 3-(anthracen9-yl)-1-(4-chlorophenyl)prop-2-en-1-one (1) as a prototype for the prepared chalcones showed a signal at $188.5 \mathrm{ppm}$ corresponding to the $\mathrm{C}=\mathrm{O}$ group.

The compounds 1-3 were converted into the corresponding 5-(anthracen-9-yl)-3-aryl-4, 5-dihydro$1 H$-pyrazole-1-carbaldehydes (4-6) by treatment with hydrazine hydrate in formic acid. The IR spectra of aldehydes 4-6 showed the $\mathrm{C}=\mathrm{O}$ band at $1666-1674 \mathrm{~cm}^{-1}$, and their proton NMR spectra showed three signals within the ranges $\delta$ 3.28-3.42, 3.85-4.09, and 6.40-6.96 ppm due to $\mathrm{H}_{4}, \mathrm{H}_{4}$, and $\mathrm{H}_{5}$ of the pyrazoline ring, respectively, in addition to a singlet signal in the range $\delta 8.84-9.00 \mathrm{ppm}$ due to the $\mathrm{CHO}$ proton.

Reaction of compounds $\mathbf{4}$ and $\mathbf{5}$ with benzoyl hydrazine gave rise to the corresponding benzoylhydrazides 7 and 8, respectively. Their IR spectra showed the NH bands at 3255,3267 , and the carbonyl absorption bands at 1659 , $1662 \mathrm{~cm}^{-1}$, respectively. Further, in their proton NMR spectra, it were observed the appearance of signals in the ranges $\delta 3.38-3.59,3.85-4.53$ and 6.71-7.86 ppm, due to $\mathrm{H}_{4}, \mathrm{H}_{4}$, and $\mathrm{H}_{5}$ of the pyrazoline ring, respectively, a singlet at $\delta 8.04$ and $8.41 \mathrm{ppm}$ due to the $\mathrm{N}_{1} \mathrm{CH}=\mathrm{N}$ protons, and a singlet at $\delta 10.48$ and $10.47 \mathrm{ppm}$, corresponding to the NH protons respectively.

The 1-(5-(anthracen-9-yl)-3-aryl-4, 5-dihydro$1 H$-pyrazol-1-yl)ethanones 9-11 were synthesized by cyclization of chalcones 1-3 with hydrazine hydrate in acetic acid. Their structures were confirmed by IR spectra, which showed their carbonyl band in the range $1655-1667 \mathrm{~cm}^{-1}$. On the other hand, their proton NMR showed a new singlet in the range $\delta 2.15-2.37 \mathrm{ppm}$, attributable to the $\mathrm{CH}_{3}$ protons, three doublets of doublets in the range $\delta 3.27-3.48$, 3.85-4.03, and 6.66-6.85 ppm corresponding to $\mathrm{H}_{4}, \mathrm{H}_{4}$, and $\mathrm{H}_{5}$ of the pyrazoline ring, respectively.
Treatment of the methyl ketones 9 and $\mathbf{1 0}$ with benzaldehyde in alkaline medium at room temperature afforded the corresponding $\alpha, \beta$-unsaturated ketones 12 and 13, respectively, the IR spectra of which showed the carbonyl group at 1650 and $1652 \mathrm{~cm}^{-1}$. Their proton NMR spectra showed the disappearance of the $\mathrm{CH}_{3}$ signals and exhibited pairs of signals at $\delta 7.40,8.67$ and 7.28, 8.70 ppm, respectively, as doublets, due to the olefinic protons $\left(\mathrm{H}_{1}, \mathrm{H}_{2}\right)$.

The desired Schiff's bases 14-19 were prepared by heating the methyl ketones 9-11 with aryl hydrazines (phenyl hydrazine, $p$-nitrophenyl hydrazine) or hydrazine hydrate in ethanol. Their IR spectra showed a new absorption peak at $3285-3372 \mathrm{~cm}^{-1}$ due to the NH group, while their ${ }^{1} \mathrm{H}$ NMR spectra displayed the $\mathrm{CH}_{3}$ protons as singlets in the range $\delta 2.14-2.19 \mathrm{ppm}$. In addition, a broad singlet was observed in the range $\delta 9.20-10.61 \mathrm{ppm}$, corresponding to the NH moiety. In case of compound $\mathbf{1 9}$, the signal of the $\mathrm{NH}_{2}$ group appeared at $\delta 7.22 \mathrm{ppm}$.

Heating the substituted 4,5-dihydro- $1 H$-pyrazole derivative 14 with acetic anhydride afforded the corresponding tetrazole 20, which evidenced disappearance of NH signals in its IR and proton NMR spectra.

Treatment of the 3-(anthracen-9-yl)-1-arylprop-2en-1-ones 1 or 2 with $p$-bromophenyl- or 2-naphthyl hydrazine hydrochloride afforded the corresponding 1,3,5-trisubstituted pyrazolines (21-23). Their ${ }^{1} \mathrm{H}$ NMR spectra showed three multiplets in the ranges $\delta$ 2.99-3.39, 3.78-4.09 and 6.86-6.98 ppm due to the pyrazoline protons, in addition to aromatic protons at $\delta 7.03-8.54 \mathrm{ppm}$.

Cyclization of the chalcones $\mathbf{1}$ and $\mathbf{2}$ with hydrazine hydrate in ethanol gave the 5-(anthracen-9-yl)-3-aryl-4, 5-dihydro- $1 H$-pyrazoles $\mathbf{2 4}$ and $\mathbf{2 5}$, respectively. Their IR spectra exhibited a $\mathrm{NH}$ absorption peak in the range 3254-3284 $\mathrm{cm}^{-1}$. On other hand, their ${ }^{1} \mathrm{H}$ NMR showed two multiplets at $\delta 3.42-4.11$ and 6.60-6.74 ppm, due to the pyrazoline protons. The NH protons were observed at $\delta 8.56$ and $9.63 \mathrm{ppm}$, respectively.

Furthermore, the disubstituted pyrazolines $\mathbf{2 4}$ and $\mathbf{2 5}$ were allowed to react with sodium nitrite, phenylisothiocyanate, and $p$-toluenesulfonyl chloride to correspondingly furnish 5-(anthracen-9-yl)-3-aryl-1nitroso-4,5-dihydro- $1 H$-pyrazoles 26 and 27, 5-(anthracen9-yl)-3-aryl- $N$-phenyl-4,5-dihydro- $1 H$-pyrazole- 1 carbothioamides $\mathbf{2 8}$ and $\mathbf{2 9}$ and 5-(anthracen-9-yl)-3-aryl1-tosyl-4,5-dihydro- $1 H$-pyrazoles 30 and 31, respectively, in $74-89 \%$ yield.

On the other hand, the reaction of chalcones 1-3 with an alcoholic solution of guanidine carbonate containing aqueous $\mathrm{NaOH}$ produced the corresponding 2-amino-4, 6-diarylpyrimidines 32-34 (Scheme 2). This transformation might proceed either by 1,4 -addition or 1,2 -addition of the 
<smiles>O=Cc1c2ccccc2cc2ccccc12</smiles>

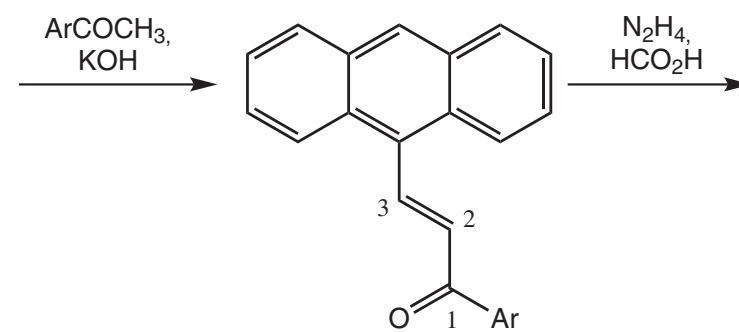<smiles>O=CN1N=C(Br)CC1c1c2ccccc2cc2ccccc12</smiles>

$1 \mathrm{Ar}=4 \mathrm{Cl}-\mathrm{C}_{6} \mathrm{H}_{4}$<smiles>CC(=O)N1N=C(Br)CC1c1c2ccccc2cc2ccccc12</smiles>

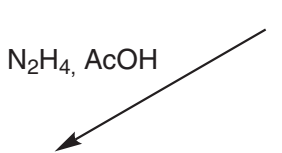

$2 \mathrm{Ar}=4 \mathrm{Me}-\mathrm{C}_{6} \mathrm{H}_{4}$ $3 \mathrm{Ar}=$<smiles>Cc1ccoc1</smiles>

$4 \mathrm{Ar}=4 \mathrm{Cl}-\mathrm{C}_{6} \mathrm{H}_{4}$

$5 \mathrm{Ar}=4 \mathrm{Me}-\mathrm{C}_{6} \mathrm{H}_{4}$

$6 \mathrm{Ar}=$<smiles>Cc1ccco1</smiles><smiles></smiles>

$10 \mathrm{Ar}=4 \mathrm{Me}-\mathrm{C}_{6} \mathrm{H}_{4}$

$11 \mathrm{Ar}=$<smiles>Cc1ccco1</smiles>

$21 \mathrm{Ar}=4 \mathrm{Cl}-\mathrm{C}_{6} \mathrm{H}_{4}, \mathrm{R}=4 \mathrm{Br}-\mathrm{C}_{6} \mathrm{H}_{4}$ $22 \mathrm{Ar}=4 \mathrm{Me}-\mathrm{C}_{6} \mathrm{H}_{4}, \mathrm{R}=4 \mathrm{Br}-\mathrm{C}_{6} \mathrm{H}_{4}$<smiles>O=C(/[Te]=C\c1ccccc1)N1N=C(Br)CC1c1c2ccccc2cc2ccccc12</smiles>
$23 \mathrm{Ar}=4 \mathrm{Me}-\mathrm{C}_{6} \mathrm{H}_{4}, \mathrm{R}=2$-Naphth

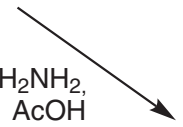<smiles>C/C(=N/N(N)P)N1N=C(Br)CC1c1c2ccccc2cc2ccccc12</smiles>

$12 \mathrm{Ar}=4 \mathrm{Cl}-\mathrm{C}_{6} \mathrm{H}_{4}$ $13 \mathrm{Ar}=4 \mathrm{Me}-\mathrm{C}_{6} \mathrm{H}_{4}$

$$
\begin{aligned}
& 14 \mathrm{Ar}=4 \mathrm{Cl}-\mathrm{C}_{6} \mathrm{H}_{4}, \mathrm{R}=\mathrm{C}_{6} \mathrm{H}_{5} \\
& 15 \mathrm{Ar}=4 \mathrm{Me}-\mathrm{C}_{6} \mathrm{H}_{4}, \mathrm{R}=\mathrm{C}_{6} \mathrm{H}_{5} \\
& 16 \mathrm{Ar}=4 \mathrm{Cl}-\mathrm{C}_{6} \mathrm{H}_{4}, \mathrm{R}=4 \mathrm{NO}_{2}-\mathrm{C}_{6} \mathrm{H}_{4} \\
& 17 \mathrm{Ar}=4 \mathrm{Me}-\mathrm{C}_{6} \mathrm{H}_{4}, \mathrm{R}=4 \mathrm{NO}_{2}-\mathrm{C}_{6} \mathrm{H}_{4} \\
& 18 \mathrm{Ar}= \\
& 19 \mathrm{Ar}=4 \mathrm{Cl}_{-} \mathrm{C}_{6} \mathrm{H}_{4}, \mathrm{R}=\mathrm{H}
\end{aligned}
$$<smiles>CCN1N=C(C)N2C(COC(=O)O)=CC(c3ccccc3)N12</smiles>

$20 \mathrm{Ar}=4 \mathrm{Cl}-\mathrm{C}_{6} \mathrm{H}_{4}, \mathrm{R}=\mathrm{C}_{6} \mathrm{H}_{5}$

Scheme 1.

guanidine to the chalcones, followed by cyclization of the intermediate, which undergoes proton shift and aromatization to yield the 2-aminopyrimidines..$^{32}$ The infrared spectra of the products showed two bands in the ranges 3054-3147 and $3323-3366 \mathrm{~cm}^{-1}$ corresponding to the $\mathrm{NH}_{2}$ group. Furthermore, their ${ }^{1} \mathrm{H}$ NMR showed a $\mathrm{D}_{2} \mathrm{O}$ exchangeable signal at $\delta$ 5.97-6.26 ppm due to the $\mathrm{NH}_{2}$ protons. A singlet at $\delta$ 7.35-7.91 ppm was observed due to the pyrimidine- $\mathrm{H}_{5}$.
Acetylation of the 2-aminopyrimidines $\mathbf{3 2}$ and $\mathbf{3 3}$ with $\mathrm{Ac}_{2} \mathrm{O}$ yielded the monoacetylated compounds $\mathbf{3 5}$ and 36, respectively. Their infrared spectra showed peaks at 3260 and $3218 \mathrm{~cm}^{-1}$ corresponding to the NH group and strong sharp peaks at 1667 and $1669 \mathrm{~cm}^{-1}$, respectively, due to the $\mathrm{C}=\mathrm{O}$ group. On the other hand, their ${ }^{1} \mathrm{H}$ NMR showed the $\mathrm{CH}_{3}$ protons as singlets at $\delta 2.25$ and $2.29 \mathrm{ppm}$, in addition to the NH protons at $\delta 10.77$ and $10.47 \mathrm{ppm}$ respectively. 


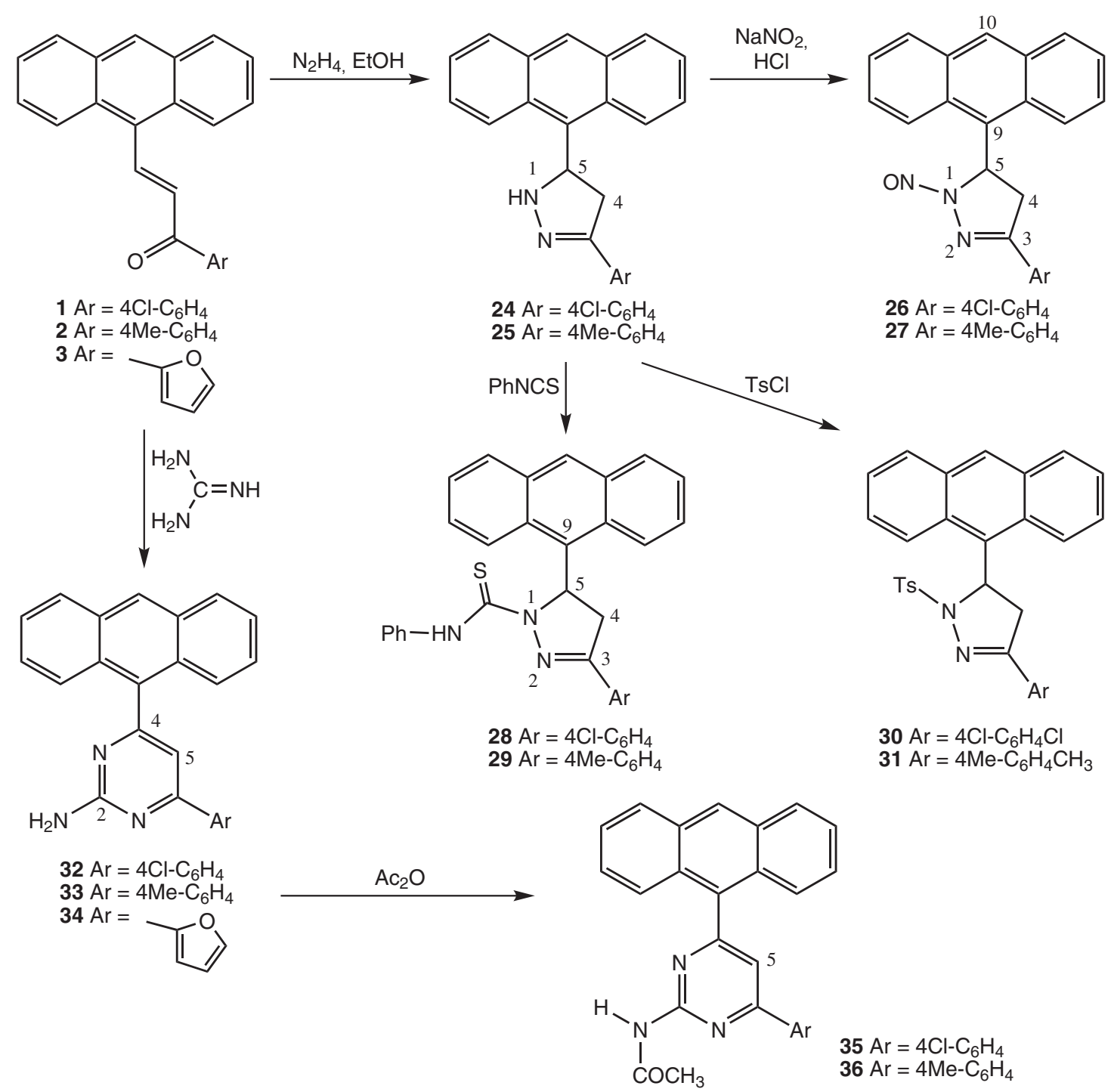

Scheme 2.

\section{Evaluation of the biological activity}

Four test organisms representing different groups of microorganisms were used to evaluate the bioactivity of the designed products. The inhibition zone and minimal inhibitory concentration results are given in Table 1.

From the data, it stems that compounds 1, 3, 7, 20, 23, 31 and $\mathbf{3 4}$ were the most active against $E$. coli, while compounds 18,28 and 30 were found to be active against C. albicans. Some chlorinated compounds exhibited activity against $C$. albicans (28 and $\mathbf{3 0}$ ) and against E. coli (1, 7 and 20). In addition, some sulfur-containing compounds exhibited activity against $C$. albicans $(\mathbf{2 8}$ and $\mathbf{3 0}$ ) and against $E$. coli (31). It was noticed that furan derivatives also showed activity against $C$. albicans $(\mathbf{1 8})$, and against $E$. coli (3 and $\mathbf{3 4})$. No systematic variation was observed in the antibacterial and antifungal activities for the rest of the compounds. All tested compounds showed poor biological activity against $P$. aeruginosa and $S$. aureus.

\section{Conclusions}

In summary, new series of anthracenylpyrazolines and anthracenylpyrimidines were synthesized from 3-(anthracen-9-yl)-1-aryl-prop-2-en-1-one derivatives, and spectroscopically characterized. The biological activity of the compounds was evaluated against $E$. coli, $P$. aeruginosa, $S$. aureus and $C$. albicans by the agar diffusion method. The potency of compounds 18, 28 and $\mathbf{3 0}$ as antifungics against $C$. albicans is about $50 \%$ of that of Clotrimazole. On the other hand, the potency of compounds $\mathbf{1}, \mathbf{3}, \mathbf{7}, \mathbf{2 0}, \mathbf{2 3}, \mathbf{3 1}$ and $\mathbf{3 4}$ as antibacterials against $E$. coli is about $50 \%$ of that of Ampicillin. 
Table 1. In vitro antimicrobial activity of the test compounds and evaluation of their antimicrobial activity of the inhibition zone (IZ) and the minimal inhibitory concentration (MIC)

\begin{tabular}{|c|c|c|c|c|c|c|c|c|}
\hline \multirow[t]{2}{*}{ Compound / Microorganism } & \multicolumn{2}{|c|}{ E. coli } & \multicolumn{2}{|c|}{ S. aureus } & \multicolumn{2}{|c|}{ C. albicans } & \multicolumn{2}{|c|}{ P. aeruginosa } \\
\hline & $\mathrm{IZ}$ & MIC & $\mathrm{IZ}$ & MIC & IZ & MIC & $\mathrm{IZ}$ & MIC \\
\hline Ampicillin $10 \mu \mathrm{g}$ per disc & 18 & 25 & 22 & 12.5 & ----- & ----- & ----- & ----- \\
\hline Ciprofloxacin $5 \mu \mathrm{g}$ per disc & 28 & 12.5 & 30 & 25 & ----- & ----- & 38 & 25 \\
\hline Clotrimazole $100 \mu \mathrm{g}$ per disc & ---- & ----- & ----- & ----- & 40 & 12.5 & ---- & $-\cdots-$ \\
\hline Imipenem $10 \mu \mathrm{g}$ per disc & 26 & ----- & 30 & ------ & ----- & ---- & 30 & ------ \\
\hline 1 & 20 & 50 & 14 & 200 & 15 & 100 & 22 & 200 \\
\hline 2 & 20 & 100 & 14 & 100 & 13 & 100 & 22 & 100 \\
\hline 3 & 15 & 50 & 10 & 200 & 13 & 100 & 18 & 200 \\
\hline 4 & 21 & 100 & 14 & 100 & 12 & 100 & 22 & 200 \\
\hline 5 & 20 & 100 & 14 & 200 & 12 & 50 & 22 & 100 \\
\hline 6 & 15 & 100 & 12 & 200 & 12 & 100 & 16 & 200 \\
\hline 7 & 15 & 50 & 10 & 200 & 12 & 100 & 17 & 100 \\
\hline 8 & 21 & 100 & 13 & 200 & 15 & 100 & 22 & 200 \\
\hline 9 & 20 & 100 & 14 & 200 & 12 & 50 & 22 & 200 \\
\hline 10 & 20 & 100 & 14 & 100 & 12 & 100 & 22 & 100 \\
\hline 11 & 15 & 100 & 12 & 200 & 14 & 100 & 17 & 200 \\
\hline 12 & 22 & 100 & 14 & 100 & 12 & 100 & 22 & 100 \\
\hline 13 & 20 & 100 & 13 & 100 & 12 & 100 & 22 & 100 \\
\hline 14 & 20 & 100 & 13 & 100 & 12 & 100 & 22 & 200 \\
\hline 15 & 20 & 100 & 13 & 200 & 13 & 100 & 22 & 200 \\
\hline 16 & 20 & 100 & 14 & 100 & 12 & 100 & 22 & 2000 \\
\hline 17 & 20 & 100 & 13 & 100 & 16 & 100 & 22 & 200 \\
\hline 18 & 16 & 100 & 13 & 200 & 13 & 25 & 17 & 100 \\
\hline 19 & 15 & 100 & 13 & 200 & 15 & 100 & 20 & 200 \\
\hline 20 & 15 & 50 & 11 & 100 & 13 & 100 & 17 & 200 \\
\hline 21 & 20 & 100 & 14 & 200 & 11 & 100 & 22 & 100 \\
\hline 22 & 22 & 100 & 14 & 100 & 12 & 100 & 22 & 200 \\
\hline 23 & 22 & 50 & 13 & 100 & 19 & 100 & 22 & 200 \\
\hline 24 & 20 & 100 & 14 & 200 & 15 & 100 & 22 & 100 \\
\hline 25 & 20 & 100 & 13 & 100 & 12 & 100 & 22 & 200 \\
\hline 26 & 16 & 100 & 12 & 200 & 13 & 100 & 17 & 100 \\
\hline 27 & 16 & 100 & 12 & 100 & 14 & 100 & 17 & 200 \\
\hline 28 & 15 & 100 & 11 & 200 & 13 & 25 & 17 & 100 \\
\hline 29 & 15 & 100 & 12 & 200 & 13 & 100 & 17 & 200 \\
\hline 30 & 16 & 100 & 12 & 200 & 14 & 25 & 19 & 100 \\
\hline 31 & 15 & 50 & 12 & 100 & 14 & 100 & 17 & 100 \\
\hline 32 & 15 & 100 & 13 & 200 & 15 & 100 & 20 & 100 \\
\hline 33 & 16 & 100 & 13 & 100 & 15 & 100 & 20 & 200 \\
\hline 34 & 16 & 50 & 14 & 200 & 15 & 100 & 20 & 200 \\
\hline 35 & 15 & 100 & 13 & 100 & 15 & 100 & 20 & 200 \\
\hline 36 & 16 & 100 & 13 & 200 & 15 & 50 & 20 & 200 \\
\hline
\end{tabular}




\section{Experimental}

\section{Chemistry}

\section{General experimental considerations}

Reagent quality solvents were used without purification. Melting points were obtained in open capillary tubes by using a MEL-Temp II melting point apparatus and are uncorrected. Infrared spectra (IR) were recorded on a Perkin-Elmer 1600 series Fourier transform instrument with the samples as $\mathrm{KBr}$ pellets. ${ }^{1} \mathrm{H} \mathrm{NMR}$ and ${ }^{13} \mathrm{C} \mathrm{NMR}$ spectra were recorded on JEOL $500 \mathrm{MHz}$ spectrometers at ambient temperature using tetramethylsilane as an internal reference. Mass spectra were recorded on a JEOL JMS AX-500 spectrometer by using electron impact ionization at $70 \mathrm{eV}$. Elemental analyses were carried out by the University of Cairo Microanalytical Laboratories. The antimicrobial tests were carried out at the Pharmaceutical Department, Faculty of Pharmacy, Alexandria University. ChemDraw-Ultra-11.0 has been used for the nomenclature of the prepared compounds.

\section{General procedure for the preparation of compounds 1-3}

An equimolar mixture of anthracene-9-carbaldehyde (2.06 $\mathrm{g}, 0.01 \mathrm{~mol})$ and the substituted ketone $(1.54 \mathrm{~g}$ 4-chloro acetophenone, $1.34 \mathrm{~g}$ 4-methyl acetophenone or $1.10 \mathrm{~g}$ 2-acetylfuran) in $2 \%$ ethanolic $\mathrm{KOH}(20 \mathrm{~mL})$ was stirred at r.t. for $4 \mathrm{~h}$. The solid product was cooled, collected by filtration, washed with ethanol, dried and recrystallized from chloroform/ethanol.

3-(Anthracen-9-yl)-1-(4-chlorophenyl)prop-2-en-1-one (1)

Yield: $90 \%$, orange-yellow crystals, mp 124-125 ${ }^{\circ} \mathrm{C}$. IR $(\mathrm{KBr}) v_{\max } / \mathrm{cm}^{-1}: 1593\left(\mathrm{C}_{2}=\mathrm{C}_{3}\right), 1656(\mathrm{C}=\mathrm{O}) ;{ }^{1} \mathrm{H}$ NMR $\left(\mathrm{CDCl}_{3}\right) \delta 7.45-7.51(\mathrm{~m}, 7 \mathrm{H},=\mathrm{CH}-2$ and $6 \mathrm{ArH}), 8.00$ (d, 4H, $J 8 \mathrm{~Hz}, \mathrm{ArH}), 8.27$ (d, 2H, $J 8 \mathrm{~Hz}, \mathrm{ArH}), 8.42$ $\left(\mathrm{s}, 1 \mathrm{H}, \mathrm{H}_{\text {anth-10 }}\right), 8.79(\mathrm{~d}, 1 \mathrm{H}, J 16 \mathrm{~Hz},=\mathrm{CH}-3) ;{ }^{13} \mathrm{C} \mathrm{NMR}$ $\left(\mathrm{DMSO}-d_{6}\right) \delta 125.2,125.4,125.5,125.6,126.6,126.7$, $128.7,128.8,129.0,129.2,129.3,129.5,129.7,130.0$, $130.1,130.3,130.6,131.4,136.3,139.7,142.5,142.6$, $188.5(\mathrm{C}=\mathrm{O})$. Anal. Calc. for $\mathrm{C}_{23} \mathrm{H}_{15} \mathrm{ClO}$ (342.08): C, 80.58; H, 4.41. Found: C, 80.63; H, 4.57\%.

\section{3-(Anthracen-9-yl)-1-p-tolylprop-2-en-1-one (2)}

Yield: $86 \%$, yellow crystals, mp $111-112^{\circ} \mathrm{C}$. IR (KBr) $\mathrm{v}_{\max } / \mathrm{cm}^{-1}: 1601\left(\mathrm{C}_{2}=\mathrm{C}_{3}\right), 1660(\mathrm{C}=\mathrm{O}) ;{ }^{1} \mathrm{H}$ NMR (DMSO- $\left.d_{6}\right)$ $\delta 2.19\left(3 \mathrm{H}, \mathrm{s}, p-\mathrm{CH}_{3}\right), 6.94$ (d, 2H, J $\left.8.0 \mathrm{~Hz}, \mathrm{ArH}\right), 7.31$ (d, 2H, J 8.0 Hz, ArH;), 7.49-7.54 (m, 4H, ArH), 7.83 (d, 1H, $J 16 \mathrm{~Hz},=\mathrm{CH}-2), 8.03(\mathrm{t}, 2 \mathrm{H}, J 7.0 \mathrm{~Hz}, \mathrm{ArH} ;), 8.31(\mathrm{~d}, 2 \mathrm{H}$, $J 7.0 \mathrm{~Hz}, \mathrm{ArH}), 8.47$ (s, 1H, $\mathrm{H}_{\text {anth-10 }}$ ), 8.79 (d, 1H, J $16 \mathrm{~Hz}$,
$=\mathrm{CH}-3)$. Anal. Calc. for $\mathrm{C}_{24} \mathrm{H}_{18} \mathrm{O}$ (322.40): C, 89.41; H, 5.63. Found: C, 89.60; H, 5.70\%.

3-(Anthracen-9-yl)-1-(furan-2-yl)prop-2-en-1-one (3)

Yield: $87 \%$, yellow crystals, mp 140-141 ${ }^{\circ} \mathrm{C}$. IR $(\mathrm{KBr}) v_{\max } / \mathrm{cm}^{-1}: 1594\left(\mathrm{C}_{2}=\mathrm{C}_{3}\right), 1651(\mathrm{C}=\mathrm{O}) ;{ }^{1} \mathrm{H}$ NMR $\left(\mathrm{DMSO}-d_{6}\right) \delta 6.75(\mathrm{t}, 1 \mathrm{H}, J 8.0 \mathrm{~Hz}, \mathrm{ArH}), 7.50-7.58(\mathrm{~m}$, $5 \mathrm{H},=\mathrm{CH}-2+4 \mathrm{ArH}), 7.74(\mathrm{~d}, 1 \mathrm{H}, J 3.0 \mathrm{~Hz}, \mathrm{ArH}), 8.12$ (d, 3H, J $8.0 \mathrm{~Hz}, \mathrm{ArH}) 8.20$ (d, 2H, J 8.0 Hz, ArH), 8.60 (d, $1 \mathrm{H}, J 16 \mathrm{~Hz},=\mathrm{CH}-3), 8.66\left(\mathrm{~s}, 1 \mathrm{H}, \mathrm{H}_{\text {anth-10 }}\right.$ ). Anal. Calc. for $\mathrm{C}_{21} \mathrm{H}_{14} \mathrm{O}_{2}$ (298.33): C, 84.54; $\mathrm{H}, 4.73$. Found: C, 84.65; $\mathrm{H}, 4.81 \%$.

\section{General procedure for the preparation of compounds 4-6}

A mixture of 3-(anthracen-9-yl)-1-(aryl)prop-2-en1-one $(\mathbf{1 - 3}, 0.001 \mathrm{~mol})$ and hydrazine hydrate $(3 \mathrm{~mL})$ in formic acid $(15 \mathrm{~mL})$ was heated under reflux for $8 \mathrm{~h}$. The reaction mixture was poured onto crushed ice, the precipitated product was filtered, washed with water, dried and recrystallized from chloroform/ethanol.

5-(Anthracen-9-yl)-3-(4-chlorophenyl)-4, 5-dihydro-1Hpyrazole-1-carbaldehyde (4)

Yield: $85 \%$, buff crystals, mp $225-226^{\circ} \mathrm{C}$. IR (KBr) $\mathrm{v}_{\max } / \mathrm{cm}^{-1}: 1674(\mathrm{C}=\mathrm{O}) ;{ }^{1} \mathrm{H}$ NMR (DMSO- $\left.d_{6}\right) \delta$ 3.36-3.39 (m, $1 \mathrm{H}$, pyrazoline- $\left.\mathrm{H}_{4}\right), 3.85-4.09\left(\mathrm{~m}, 1 \mathrm{H}\right.$, pyrazoline- $\left.\mathrm{H}_{4}\right)$, 6.86-6.96 (dd, 1H, $J_{1,2} 13, J_{1,3} 18 \mathrm{~Hz}$, pyrazoline- $\left.\mathrm{H}_{5}\right), 7.48$ (dd, 2H, J 3, 7.0 Hz, ArH), 7.56 (d, 2H, J 7.6 Hz, ArH), 7.69 (d, 2H, J 7.0 Hz, ArH), 7.91(d, 2H, J 7.0 Hz, ArH), 8.05 (dd, 2H, J 3.0, 7.0 Hz, ArH), 8.11 (d, 2H, J 7.6 Hz, ArH), $8.53\left(\mathrm{~s}, 1 \mathrm{H}, \mathrm{H}_{\text {anth-10 }}\right), 8.84$ (s, 1H, CHO). Anal. Calc. for $\mathrm{C}_{24} \mathrm{H}_{17} \mathrm{ClN}_{2} \mathrm{O}$ (384.86): C, 74.90; H, 4.45; N, 7.28. Found: C, $74.65 ; \mathrm{H}, 4.23 ; \mathrm{N}, 7.49 \%$.

5-(Anthracen-9-yl)-3-p-tolyl-4,5-dihydro-1H-pyrazole-1carbaldehyde (5)

Yield: $81 \%$, brown crystals, mp $199-200{ }^{\circ} \mathrm{C}$. IR (KBr) $v_{\max } / \mathrm{cm}^{-1}: 1666(\mathrm{C}=\mathrm{O}) ;{ }^{1} \mathrm{H}$ NMR (DMSO-d $)_{6} \delta 2.19(3 \mathrm{H}$, $\left.\mathrm{s}, p-\mathrm{CH}_{3}\right), 3.15-3.30\left(1 \mathrm{H}, \mathrm{m}\right.$, pyrazoline- $\left.\mathrm{H}_{4}\right), 4.08(\mathrm{dd}$, $1 \mathrm{H}, J_{1,2} 13, J_{1,3} 18 \mathrm{~Hz}$, pyrazoline- $\left.\mathrm{H}_{4}\right), 6.88(\mathrm{t}, 1 \mathrm{H}, J$ 7.0, $12 \mathrm{~Hz}$, pyrazoline- $\left.\mathrm{H}_{5}\right), 7.37-7.58(\mathrm{~m}, 6 \mathrm{H}, \mathrm{ArH}), 7.67$ (d, $1 \mathrm{H}, J 8.0 \mathrm{~Hz}, \mathrm{ArH}), 7.86$ (d, 2H, J 7.6 Hz, ArH), 8.00 (d, $2 \mathrm{H}, J$ 8.0 Hz, ArH), 8.56-8.58 (m, 2H, ArH), 9.00 (s, 1H, CHO). Anal. Calc. for $\mathrm{C}_{25} \mathrm{H}_{20} \mathrm{~N}_{2} \mathrm{O}$ (364.44): C, 82.39; $\mathrm{H}$, 5.53; N, 7.69. Found: C, 82.15; H, 5.33; N, 7.90\%.

5-(Anthracen-9-yl)-3-(furan-2-yl)-4, 5-dihydro-1Hpyrazole-1-carbaldehyde (6)

Yield: $78 \%$, buff crystals, mp $204-205{ }^{\circ} \mathrm{C}$. IR (KBr) $v_{\max } / \mathrm{cm}^{-1}: 1670(\mathrm{C}=\mathrm{O}) ;{ }^{1} \mathrm{H}$ NMR (DMSO- $\left.d_{6}\right) \delta 3.28-3.42$ 
(m, 1H, pyrazoline- $\left.\mathrm{H}_{4}\right), 3.98-4.07\left(\mathrm{~m}, 1 \mathrm{H}\right.$, pyrazoline- $\left.\mathrm{H}_{4}\right)$, 6.40-6.48 (m, 1H, pyrazoline- $\left.\mathrm{H}_{5}\right), 6.54(\mathrm{~d}, 1 \mathrm{H}, J 3.0 \mathrm{~Hz}$, ArH), 6.70 (t, 1H, J 8.0 Hz, ArH), 6.86 (d, 2H, J 7.0 Hz, ArH), 7.05 (d, 2H, J 7.0 Hz, ArH), 7.20 (d, 1H, J 8.0 Hz, ArH), 7.44 (dd, 2H, J3.0, 7.0 Hz, ArH), 8.03 (dd, 2H, J3.0, $7.0 \mathrm{~Hz}, \mathrm{Ar}-\mathrm{H}), 8.52$ (s, 1H, $\mathrm{H}_{\text {anth-10 }}$ ), 8.59 (s, 1H, CHO). Anal. Calc. for $\mathrm{C}_{22} \mathrm{H}_{16} \mathrm{~N}_{2} \mathrm{O}_{2}(340.37)$ : C, 77.63; H, 4.74; N, 8.23. Found: C, 77.69; H, 4.83; N, 8.04\%.

General procedure for the preparation of compounds 7 and 8

To a solution of 5-(anthracen-9-yl)-3-aryl-4, 5-dihydro$1 H$-pyrazole-1-carbaldehyde 4 (3.84 g, $0.01 \mathrm{~mol})$ or 5 $(3.64 \mathrm{~g}, 0.01 \mathrm{~mol})$ in ethanol $(30 \mathrm{~mL})$ was added benzoyl hydrazine (1.63 g, $0.012 \mathrm{~mol})$ and two drops of acetic acid. The reaction mixture was heated under reflux for $6 \mathrm{~h}$, partially concentrated and cooled. The separated solid product was filtered, washed with ethanol, dried and crystallized from ethanol.

N'-((5-(Anthracen-9-yl)-3-(4-chlorophenyl)-4,5-dihydro1H-pyrazole-1-yl)methylene) benzoylhydrazide (7)

Yield: $77 \%$, buff crystals, mp 207-208 ${ }^{\circ} \mathrm{C}$. IR (KBr) $v_{\max } / \mathrm{cm}^{-1}: 1659(\mathrm{C}=\mathrm{O}), 3255(\mathrm{NH}) ;{ }^{1} \mathrm{H}$ NMR (DMSO- $\left.d_{6}\right)$ $\delta$ 3.38-3.59 (m, 1H, pyrazoline- $\left.\mathrm{H}_{4}\right), 4.51-4.53(\mathrm{~m}, 1 \mathrm{H}$, pyrazoline- $\left.\mathrm{H}_{4}\right), 6.71-6.86\left(\mathrm{~m}, 1 \mathrm{H}\right.$, pyrazoline- $\left.\mathrm{H}_{5}\right), 7.31$ 7.37 (m, 4H, ArH), 7.47-7.62 (m, 2H, ArH), 7.71-7.83 (m, $5 \mathrm{H}, \mathrm{ArH}), 7.89-7.96$ (m, 4H, ArH), 8.04 (s, 1H, $\left.\mathrm{N}_{1} \mathrm{CH}=\mathrm{N}\right)$, 8.17-8.32 (m, 2H, ArH), 8.52 (s, 1H, $\mathrm{H}_{\text {anth- } 10}$ ), 10.48 (s, 1H, $\mathrm{NH} ; \mathrm{D}_{2} \mathrm{O}$ exchangeable). Anal. Calc. for $\mathrm{C}_{31} \mathrm{H}_{23} \mathrm{ClN}_{4} \mathrm{O}$ (502.99): C, 74.02; H, 4.61; N, 11.14. Found: C, 74.24; $\mathrm{H}, 4.85 ; \mathrm{N}, 11.00 \%$.

$N^{\prime}-((5-($ Anthracen-9-yl)-3-p-tolyl-4,5-dihydro- $1 H$ pyrazole-1-yl)methylene)benzoylhydrazide (8)

Yield: $74 \%$, buff crystals, mp $181-182{ }^{\circ} \mathrm{C}$. IR (KBr) $v_{\max } / \mathrm{cm}^{-1}: 1662(\mathrm{C}=\mathrm{O}), 3267(\mathrm{NH}) ;{ }^{1} \mathrm{H} \mathrm{NMR}\left(\mathrm{CDCl}_{3}\right) \delta 2.18$ $\left(3 \mathrm{H}, \mathrm{s}, p-\mathrm{CH}_{3}\right), 3.46$ (dd, $1 \mathrm{H}, J_{1,2} 9, J_{1,3} 18 \mathrm{~Hz}$, pyrazoline- $\mathrm{H}_{4}$ ), $3.85\left(\mathrm{dd}, 1 \mathrm{H}, J_{1,2} 13, J_{1,3} 18 \mathrm{~Hz}\right.$, pyrazoline- $\left.\mathrm{H}_{4}\right), 6.86(\mathrm{dd}, 1 \mathrm{H}$, $J_{1,2} 9, J_{1,3} 13 \mathrm{~Hz}$, pyrazoline- $\left.\mathrm{H}_{5}\right), 7.34-7.46(\mathrm{~m}, 10 \mathrm{H}, \mathrm{ArH})$, 7.73-7.81 (m, 4H, ArH), 8.00-8.10 (m, 3H, ArH), 8.41 (s, $\left.1 \mathrm{H}, \mathrm{N}_{1} \mathrm{CH}=\mathrm{N}\right), 8.50\left(\mathrm{~s}, 1 \mathrm{H}, \mathrm{H}_{\text {anth-10 }}\right), 10.47$ (s, 1H, NH; $\mathrm{D}_{2} \mathrm{O}$ exchangeable). Anal. Calc. for $\mathrm{C}_{32} \mathrm{H}_{26} \mathrm{~N}_{4} \mathrm{O}$ (482.58): C, 79.64; H, 5.43; N, 11.61. Found: C, 79.41; H, 5.60; N, $11.83 \%$.

General procedure for the preparation of compounds 9-11

A mixture of 3-(anthracen-9-yl)-1-(aryl)prop-2-en1-one $(\mathbf{1 - 3}, 0.001 \mathrm{~mol})$ and hydrazine hydrate $(3 \mathrm{~mL})$ in acetic acid $(15 \mathrm{~mL})$ was heated under reflux for $8 \mathrm{~h}$. The reaction mixture was poured onto crushed ice, the precipitated product was filtered, washed with water, dried and recrystallized from chloroform/ethanol.

1-(5-(Anthracen-9-yl)-3-(4-chlorophenyl)-4,5-dihydro1H-pyrazol-1-yl)ethanone (9)

Yield: $84 \%$, buff crystals, mp $239-240{ }^{\circ} \mathrm{C}$. IR (KBr) $\mathrm{v}_{\max } / \mathrm{cm}^{-1}: 1655(\mathrm{C}=\mathrm{O}) ;{ }^{1} \mathrm{H} \mathrm{NMR}\left(\mathrm{CDCl}_{3}\right) \delta 2.35(\mathrm{~s}, 3 \mathrm{H}$, $\left.\mathrm{COCH}_{3}\right), 3.46\left(\mathrm{dd}, 1 \mathrm{H}, J_{1,2} 9, J_{1,3} 18 \mathrm{~Hz}\right.$, pyrazoline- $\left.\mathrm{H}_{4}\right), 3.85$ $\left(\mathrm{dd}, 1 \mathrm{H}, J_{1,2} 13, J_{1,3} 18 \mathrm{~Hz}\right.$, pyrazoline- $\left.\mathrm{H}_{4}\right), 6.85\left(\mathrm{dd}, 1 \mathrm{H}, J_{1,2} 9\right.$, $J_{1,3} 13 \mathrm{~Hz}$, pyrazoline- $\mathrm{H}_{5}$ ), 7.33-7.48 (m, 5H, ArH), 7.55 (t, 1H, J 7.6 Hz, ArH), 7.74 (dd, 3H, J 2, 9 Hz, ArH), 8.01 (dd, 2H, J 3, 7.6 Hz, ArH), 8.41 (s, 1H, $\mathrm{H}_{\text {anth-10 }}$ ), 8.49 (d, $1 \mathrm{H}, J 9 \mathrm{~Hz}, \mathrm{ArH})$. Anal. Calc. for $\mathrm{C}_{25} \mathrm{H}_{19} \mathrm{ClN}_{2} \mathrm{O}$ (398.88): C, 75.28; H, 4.80; N, 7.02. Found: C, 75.08; H, 4.65; N, 7.21\%.

1-(5-(Anthracen-9-yl)-3-p-tolyl-4,5-dihydro- $1 \mathrm{H}$ pyrazol-1-yl)ethanone (10)

Yield: $78 \%$, buff crystals, mp 194-195 ${ }^{\circ} \mathrm{C}$. IR (KBr) $\mathrm{v}_{\max } / \mathrm{cm}^{-1}: 1667(\mathrm{C}=\mathrm{O}) ;{ }^{1} \mathrm{H}$ NMR $\left(\mathrm{CDCl}_{3}\right) \delta 2.37(\mathrm{~s}$, $\left.3 \mathrm{H}, \mathrm{COCH}_{3}\right), 2.43\left(\mathrm{~s}, 3 \mathrm{H}, p-\mathrm{CH}_{3}\right), 3.48\left(\mathrm{dd}, 1 \mathrm{H}, J_{1,2}\right.$ 9, $J_{1,3} 18 \mathrm{~Hz}$, pyrazoline- $\left.\mathrm{H}_{4}\right), 3.89\left(\mathrm{dd}, 1 \mathrm{H}, J_{1,2} 13, J_{1,3}\right.$ $18 \mathrm{~Hz}$, pyrazoline- $\left.\mathrm{H}_{4}\right) 6.85\left(\mathrm{dd}, 1 \mathrm{H}, J_{1,2} 9, J_{1,3} 13 \mathrm{~Hz}\right.$, pyrazoline- $\mathrm{H}_{5}$ ), 7.27 (t, 2H, J 7.6 Hz, ArH), 7.32-7.41 (m, 2H, ArH), 7.49 (t, 1H, J 7.6 Hz, ArH), 7.54-7.57 (m, 1H, ArH), 7.72 (d, 2H, J 7.6 Hz, ArH), 7.79 (d, 1H, J 8 Hz, $\operatorname{ArH}), 8.01$ (d, 2H, J $8.0 \mathrm{~Hz}, \operatorname{ArH}), 8.42$ (s, 1H, $\mathrm{H}_{\text {anth-10 }}$ ), 8.52 (d, $1 \mathrm{H}, J 8.0 \mathrm{~Hz}$, ArH). Anal. Calc.for $\mathrm{C}_{26} \mathrm{H}_{22} \mathrm{~N}_{2} \mathrm{O}$ (378.47): C, 82.51; H, 5.86; N, 7.40. Found: C, 82.72; H, $5.98 ; \mathrm{N}, 7.19 \%$.

1-(5-(Anthracen-9-yl)-3-(furan-2-yl)-4,5-dihydro- $1 \mathrm{H}$ pyrazol-1-yl)ethanone (11)

Yield: $80 \%$, buff crystals, mp $200-201{ }^{\circ} \mathrm{C}$. IR (KBr) $v_{\max } / \mathrm{cm}^{-1}: 1657(\mathrm{C}=\mathrm{O}) ;{ }^{1} \mathrm{H}$ NMR (DMSO-d $) \delta 2.15(3 \mathrm{H}$, $\mathrm{s}, \mathrm{COCH}_{3}$ ), 3.27 (dd, $1 \mathrm{H}, J_{1,2} 9, J_{1,3} 18 \mathrm{~Hz}$, pyrazoline- $\mathrm{H}_{4}$ ), 4.03 (dd, $1 \mathrm{H}, J_{1,2} 13, J_{1,3} 18 \mathrm{~Hz}$, pyrazoline- $\mathrm{H}_{4}$ ), 6.66-6.67 (m, $1 \mathrm{H}, \mathrm{ArH}), 6.84$ (dd, $1 \mathrm{H}, J_{1,2} 9, J_{1,3} 13 \mathrm{~Hz}$ pyrazoline- $\mathrm{H}_{5}$ ), 6.99 (d, 1H, J 3.0 Hz, ArH), 7.42 (t, 2H, J $8.0 \mathrm{~Hz}, \mathrm{ArH})$, 7.51 (t, 1H, J 7.0 Hz, ArH), 7.56 (t, 1H, J 7.0 Hz, ArH), 7.68 (d, 1H, J $3.0 \mathrm{~Hz}, \mathrm{ArH}), 7.90\left(\mathrm{~s}, 1 \mathrm{H}, \mathrm{H}_{\text {anth-10 }}\right), 8.07$ (d, 2H, J 7.0 Hz, ArH), 8.55-8.56 (m, 2H, ArH). Anal. Calc. for $\mathrm{C}_{23} \mathrm{H}_{18} \mathrm{~N}_{2} \mathrm{O}_{2}$ (354.40): C, 77.95; H, 5.12; N, 7.90. Found: C, 77.73; H, 5.32; N, 7.99\%.

General procedure for the preparation of compounds 12 and 13

An equimolar mixture of 1-(5-(anthracen-9-yl)-3aryl-4, 5-dihydro-1H-pyrazol-1-yl)ethanone 9 (3.98 g, 
$0.01 \mathrm{~mol})$ or $\mathbf{1 0}(3.78 \mathrm{~g}, 0.01 \mathrm{~mol})$ and benzaldehyde $(1.06 \mathrm{~g}, 0.01 \mathrm{~mol})$ in $2 \%$ ethanolic $\mathrm{KOH}(20 \mathrm{~mL})$ was stirred at r.t. for $4 \mathrm{~h}$. The solid product was cooled, collected by filtration, washed with ethanol, dried, and recrystallized from chloroform/ethanol.

1-(5-(Anthracen-9-yl)-3-(4-chlorophenyl)-4,5-dihydro1H-pyrazol-1-yl)-3-phenyl-prop-2-en-1-one (12)

Yield: $71 \%$, off white needles, mp $259-260{ }^{\circ} \mathrm{C}$. IR $(\mathrm{KBr}) v_{\max } / \mathrm{cm}^{-1}: 1650(\mathrm{C}=\mathrm{O}) ;{ }^{1} \mathrm{H}$ NMR (DMSO- $\left.d_{6}\right)$ $\delta 3.33-3.37\left(\mathrm{~m}, 1 \mathrm{H}\right.$, pyrazoline- $\left.\mathrm{H}_{4}\right), 4.07\left(\mathrm{dd}, 1 \mathrm{H}, J_{1,2} 13\right.$, $J_{l, 3} 18 \mathrm{~Hz}$, pyrazoline- $\left.\mathrm{H}_{4}{ }^{\prime}\right), 6.88\left(\mathrm{dd}, 1 \mathrm{H}, J_{1,2} 9, J_{l, 3} 13 \mathrm{~Hz}\right.$, pyrazoline- $\left.\mathrm{H}_{5}\right), 7.40(\mathrm{~d}, 1 \mathrm{H}, J 6.0 \mathrm{~Hz},=\mathrm{CH}-1),, 7.42-7.47$ (m, 4H, ArH), 7.50-7.58 (m, 5H, ArH), 7.67 (d, 2H, J 8 Hz, ArH), 7.86 (d, 2H, J 7.6 Hz, ArH), $7.96(\mathrm{~d}, 2 \mathrm{H}, J 8 \mathrm{~Hz}$, ArH), 8.08 (d, 2H, J 7.6 Hz, ArH), 8.55 (s, 1H, $\mathrm{H}_{\text {anth-10 }}$ ), $8.67(\mathrm{~d}, 1 \mathrm{H}, J 6.0 \mathrm{~Hz},=\mathrm{CH}-2)$. Anal. Calc. for $\mathrm{C}_{32} \mathrm{H}_{23} \mathrm{ClN}_{2} \mathrm{O}$ (486.99): C, 78.92; H, 4.76; N, 5.75. Found: C, 79.00; H, $4.82 ; \mathrm{N}, 5.58 \%$.

1 -(5-(Anthracen-9-yl)-3-p-tolyl-4,5-dihydro- $1 \mathrm{H}$ pyrazol-1-yl)-3-phenylprop-2-en-1-one (13)

Yield: $73 \%$, off white needles, mp $225-226^{\circ} \mathrm{C}$. IR (KBr) $v_{\max } / \mathrm{cm}^{-1}: 1652(\mathrm{C}=\mathrm{O}) ;{ }^{1} \mathrm{H}$ NMR (DMSO- $\left.d_{6}\right)$ $\delta 2.33\left(3 \mathrm{H}, \mathrm{s}, p-\mathrm{CH}_{3}\right), 3.27-3.38\left(\mathrm{~m}, 1 \mathrm{H}\right.$, pyrazoline- $\left.\mathrm{H}_{4}\right)$, $4.07\left(\mathrm{dd}, 1 \mathrm{H}, J_{1,2} 13, J_{1,3} 18 \mathrm{~Hz}\right.$, pyrazoline- $\left.\mathrm{H}_{4}\right), 6.86$ $\left(\mathrm{dd}, 1 \mathrm{H}, J_{1,2} 9, J_{1,3} 13 \mathrm{~Hz}\right.$, pyrazoline- $\left.\mathrm{H}_{5}\right), 7.28(\mathrm{~d}, 1 \mathrm{H}$, $J 6 \mathrm{~Hz},=\mathrm{CH}-1), 7.35(\mathrm{t}, 2 \mathrm{H}, J 7.6 \mathrm{~Hz}, \mathrm{ArH}), 7.41(\mathrm{t}, 2 \mathrm{H}$, $J$ 7.0 Hz, ArH), 7.50-7.58 (m, 5H, ArH), $7.70(\mathrm{~d}, 2 \mathrm{H}$, $J 7.0 \mathrm{~Hz}, \mathrm{ArH}), 7.77$ (d, 2H, J 7.6 Hz, ArH), 8.08 (d, 2H, $J 8.0 \mathrm{~Hz}, \mathrm{ArH}), 8.55\left(\mathrm{~s}, 1 \mathrm{H}, \mathrm{H}_{\text {anth-10 }}\right), 8.58(\mathrm{~d}, 2 \mathrm{H}, J 8.0 \mathrm{~Hz}$, $\mathrm{ArH}), 8.70(\mathrm{~d}, 1 \mathrm{H}, J 6.0 \mathrm{~Hz},=\mathrm{CH}-2)$. Anal. Calc. for $\mathrm{C}_{33} \mathrm{H}_{26} \mathrm{~N}_{2} \mathrm{O}$ (466.57): C, 84.95; H, 5.62; N, 6.00. Found: C, 84.70; H, 5.90; N, 6.27\%.

General procedure for the preparation of compounds 14-19

To a solution of 1-(5-(anthracen-9-yl)-3-aryl-4, 5-dihydro-1H-pyrazol-1-yl)ethanone (9-11, $0.01 \mathrm{~mol})$ in ethanol $(25 \mathrm{~mL})$ was added hydrazine hydrate or aryl hydrazine $(0.012 \mathrm{~mol})$ and two drops of acetic acid. The reaction mixture was heated under reflux for $8 \mathrm{~h}$, partially concentrated and cooled. The separated solid product was filtered, washed with ethanol, dried and crystallized from ethanol.

5-(Anthracen-9-yl)-3-(4-chlorophenyl)-1-(1-(2phenylhydrazono)ethyl)-4,5-dihydro-1H-pyrazole (14)

Yield: $77 \%$, buff crystals, mp $270-271{ }^{\circ} \mathrm{C}$. IR (KBr) $v_{\max } / \mathrm{cm}^{-1}: 3295(\mathrm{NH}) ;{ }^{1} \mathrm{H}$ NMR (DMSO- $\left.d_{6}\right) \delta 2.19(3 \mathrm{H}$, s, N=C- $\left.\mathrm{CH}_{3}\right), 3.22-3.40\left(\mathrm{~m}, 1 \mathrm{H}\right.$, pyrazoline $\left.-\mathrm{H}_{4}\right), 4.07$ $\left(\mathrm{m}, 1 \mathrm{H}\right.$, pyrazoline- $\left.\mathrm{H}_{4}\right), 6.89\left(\mathrm{dd}, 1 \mathrm{H}, J_{1,2} 9, J_{1,3} 13 \mathrm{~Hz}\right.$, pyrazoline- $\left.\mathrm{H}_{5}\right), 7.37(\mathrm{t}, 2 \mathrm{H}, J 8 \mathrm{~Hz}, \mathrm{ArH}), 7.42(\mathrm{t}, 2 \mathrm{H}$, $J 7.0 \mathrm{~Hz}, \mathrm{ArH}), 7.52-7.58(\mathrm{~m}, 5 \mathrm{H}, \mathrm{ArH}), 7.66(\mathrm{~d}, 2 \mathrm{H}$, $J 8.0 \mathrm{~Hz}, \mathrm{ArH}), 7.87$ (d, 4H, J 8.0 Hz, ArH), $8.06(\mathrm{~d}, 2 \mathrm{H}$, $J 7.0 \mathrm{~Hz}, \mathrm{ArH}), 8.56\left(\mathrm{~s}, 1 \mathrm{H}, \mathrm{H}_{\text {anth-10 }}\right), 9.58(\mathrm{~s}, 1 \mathrm{H}, \mathrm{NH}$; $\mathrm{D}_{2} \mathrm{O}$ exchangeable). Anal. Calc.for $\mathrm{C}_{31} \mathrm{H}_{25} \mathrm{ClN}_{4}$ (489.01): C, 76.14; H, 5.15; N, 11.46. Found: C, 76.31; H, 5.26; N, $11.19 \%$.

5-(Anthracen-9-yl)-1-(1-(2-phenylhydrazono)ethyl)-3-ptolyl-4,5-dihydro-1H-pyrazole (15)

Yield: $78 \%$, reddish brown crystals, mp $89-90{ }^{\circ} \mathrm{C}$. IR $(\mathrm{KBr}) v_{\max } / \mathrm{cm}^{-1}: 3297(\mathrm{NH}) ;{ }^{1} \mathrm{H}$ NMR (DMSO- $\left.d_{6}\right) \delta 2.19$ $\left(3 \mathrm{H}, \mathrm{s}, \mathrm{N}=\mathrm{C}-\mathrm{CH}_{3}\right), 2.34\left(3 \mathrm{H}, \mathrm{s}, p-\mathrm{CH}_{3}\right), 3.33-3.39(\mathrm{~m}, 1 \mathrm{H}$, pyrazoline- $\left.\mathrm{H}_{4}\right), 4.08\left(\mathrm{~m}, 1 \mathrm{H}\right.$, pyrazoline- $\left.\mathrm{H}_{4}\right), 6.87(\mathrm{dd}, 1 \mathrm{H}$, $J_{1,2} 9, J_{1,3} 13 \mathrm{~Hz}$, pyrazoline- $\left.\mathrm{H}_{5}\right), 7.21-7.77(\mathrm{~m}, 12 \mathrm{H}, \mathrm{ArH})$, 8.09 (d, 2H, J 8.0 Hz, ArH), 8.18-8.20 (m, 2H, ArH), 8.56$8.60(\mathrm{~m}, 2 \mathrm{H}, \mathrm{ArH}), 10.61$ (s, 1H, NH; $\mathrm{D}_{2} \mathrm{O}$ exchangeable). Anal. Calc.for $\mathrm{C}_{32} \mathrm{H}_{28} \mathrm{~N}_{4}$ (468.59): C, 82.02; H, 6.02; N, 11.96. Found: C, $82.25 ; \mathrm{H}, 5.88 ; \mathrm{N}, 12.20 \%$.

5-(Anthracen-9-yl)-3-(4-chlorophenyl)-1-(1-(2-(4nitrophenyl)hydrazono)ethyl)-4,5-dihydro-1H-pyrazole (16)

Yield: $79 \%$, pale brown crystals, mp $180-181{ }^{\circ} \mathrm{C}$. IR $(\mathrm{KBr}) v_{\max } / \mathrm{cm}^{-1}: 3285(\mathrm{NH}) ;{ }^{1} \mathrm{H}$ NMR (DMSO- $\left.d_{6}\right) \delta 2.19$ $\left(\mathrm{s}, 3 \mathrm{H}, \mathrm{N}=\mathrm{C}-\mathrm{CH}_{3}\right), 3.26-3.33\left(\mathrm{~m}, 1 \mathrm{H}\right.$, pyrazoline- $\left.\mathrm{H}_{4}\right), 4.08$ (dd, $1 \mathrm{H}, J_{1,2} 13, J_{1,3} 18 \mathrm{~Hz}$, pyrazoline- $\left.\mathrm{H}_{4}\right), 6.88(\mathrm{dd}, 1 \mathrm{H}$, $J_{l, 2} 9, J_{l, 3} 13 \mathrm{~Hz}$, pyrazoline- $\left.\mathrm{H}_{5}\right), 7.43-7.52(\mathrm{~m}, 4 \mathrm{H}, \mathrm{ArH})$, $7.55(\mathrm{~d}, 2 \mathrm{H}, J 9.0 \mathrm{~Hz}, \mathrm{ArH}), 7.66(\mathrm{~d}, 2 \mathrm{H}, J 9.0 \mathrm{~Hz}, \mathrm{ArH})$, 7.69-7.74 (m, 2H, ArH), 7.87 (d, 2H, J 8.0 Hz, ArH;), 7.97 (d, 2H, J 7.6 Hz, ArH), 8.08 (d, 2H, J 7.6 Hz, ArH), 8.57 (s, $1 \mathrm{H}, \mathrm{H}_{\text {anth-10 }}$ ), 9.22 (s, 1H, NH; $\mathrm{D}_{2} \mathrm{O}$ exchangeable). Anal. Calc. for $\mathrm{C}_{31} \mathrm{H}_{24} \mathrm{ClN}_{5} \mathrm{O}_{2}$ (534.01): C, 69.72; H, 4.53; N, 13.11. Found: C, 69.79; H, 4.80; N, $12.97 \%$.

5-(Anthracen-9-yl)-1-(1-(2-(4-nitrophenyl)hydrazono) ethyl)-3-p-tolyl-4,5-dihydro-1H-pyrazole (17)

Yield: $80 \%$, pale brown crystals, mp $85-86{ }^{\circ} \mathrm{C}$. IR (KBr) $v_{\max } / \mathrm{cm}^{-1}: 3300(\mathrm{NH}) ;{ }^{1} \mathrm{H}$ NMR (DMSO- $d_{6}$ ) $\delta 2.19\left(3 \mathrm{H}, \mathrm{s}, \mathrm{N}=\mathrm{C}-\mathrm{CH}_{3}\right), 2.33\left(3 \mathrm{H}, \mathrm{s}, p-\mathrm{CH}_{3}\right), 3.30-$ $3.40\left(\mathrm{~m}, 1 \mathrm{H}\right.$, pyrazoline- $\left.\mathrm{H}_{4}\right), 4.08\left(\mathrm{dd}, 1 \mathrm{H}, J_{l, 2} 13\right.$, $J_{l, 3} 18 \mathrm{~Hz}$, pyrazoline- $\left.\mathrm{H}_{4}\right), 6.86\left(\mathrm{dd}, 1 \mathrm{H}, J_{l, 2} 9, J_{l, 3} 13 \mathrm{~Hz}\right.$, pyrazoline- $\left.\mathrm{H}_{5}\right), 7.28(\mathrm{~d}, 2 \mathrm{H}, J 8.0 \mathrm{~Hz}, \mathrm{ArH}), 7.34-7.43$ (m, 3H, ArH), 7.50-7.58 (m, 3H, ArH), 7.69-7.82 (m, $4 \mathrm{H}, \mathrm{ArH}), 8.12(\mathrm{~d}, 2 \mathrm{H}, J 8.0 \mathrm{~Hz}, \mathrm{ArH}), 8.55(\mathrm{~s}, 1 \mathrm{H}$, $\left.\mathrm{H}_{\text {anth-10 }}\right), 8.58(\mathrm{~d}, 2 \mathrm{H}, J 8.0 \mathrm{~Hz}, \mathrm{ArH}), 9.20(\mathrm{~s}, 1 \mathrm{H}, \mathrm{NH}$; $\mathrm{D}_{2} \mathrm{O}$ exchangeable), MS $m / z: 513$ (M). Anal. Calc.for $\mathrm{C}_{32} \mathrm{H}_{27} \mathrm{~N}_{5} \mathrm{O}_{2}$ (513.59): C, 74.83; H, 5.30; N, 13.64. Found: C, $74.60 ; \mathrm{H}, 5.09 ; \mathrm{N}, 13.85 \%$. 
5-(Anthracen-9-yl)-3-(furan-2-yl)-1-(1-(2-(4-nitrophenyl) hydrazono)ethyl)-4,5-dihydro-1H-pyrazole (18)

Yield: $81 \%$, brown crystals, mp $120-121{ }^{\circ} \mathrm{C}$. IR $(\mathrm{KBr}) v_{\max } / \mathrm{cm}^{-1}: 1323,1502\left(\mathrm{NO}_{2}\right), 3372(\mathrm{NH}) ;{ }^{1} \mathrm{H}$ NMR $\left(\right.$ DMSO- $\left.d_{6}\right) \delta 2.14\left(3 \mathrm{H}, \mathrm{s}, \mathrm{N}=\mathrm{C}-\mathrm{CH}_{3}\right), 3.26(\mathrm{dd}, 1 \mathrm{H}$, $J_{1,2} 9, J_{1,3} 18 \mathrm{~Hz}$, pyrazoline- $\left.\mathrm{H}_{4}\right), 4.03\left(\mathrm{dd}, 1 \mathrm{H}, J_{1,2} 13\right.$, $J_{1,3} 18 \mathrm{~Hz}$, pyrazoline- $\left.\mathrm{H}_{4}\right), 6.84\left(\mathrm{dd}, 1 \mathrm{H}, J_{1,2} 9, J_{1,3} 13 \mathrm{~Hz}\right.$ pyrazoline- $\left.\mathrm{H}_{5}\right), 6.98(\mathrm{~d}, 1 \mathrm{H}, J 3.0 \mathrm{~Hz}, \mathrm{ArH}), 7.42(\mathrm{t}, 3 \mathrm{H}$, $J 3.8 \mathrm{~Hz}, \mathrm{ArH}), 7.50-7.56$ (m, 4H, ArH), 7.67 (d, 1H, $J 10$ $\mathrm{Hz}, \mathrm{ArH}), 7.93\left(\mathrm{~s}, 1 \mathrm{H}, \mathrm{H}_{\text {anth-10 }}\right), 8.07$ (d, $4 \mathrm{H}, J 7.6 \mathrm{~Hz}$, ArH), 8.54-8.56 (m, 2H, ArH), $9.20\left(\mathrm{~s}, 1 \mathrm{H}, \mathrm{NH} ; \mathrm{D}_{2} \mathrm{O}\right.$ exchangeable). Anal. Calc. for $\mathrm{C}_{29} \mathrm{H}_{23} \mathrm{~N}_{5} \mathrm{O}_{3}$ (489.52): $\mathrm{C}$, 71.15; H, 4.74; N, 14.31. Found: C, 71.33; H, 4.51; N, $14.05 \%$.

5-(Anthracen-9-yl)-3-(4-chlorophenyl)-1-(1hydrazonoethyl)-4, 5-dihydro-1H-pyrazole (19)

Yield: $85 \%$, pale yellow needles, mp $275-276{ }^{\circ} \mathrm{C}$. ${ }^{1} \mathrm{H}$ NMR (DMSO- $\left.d_{6}\right) \delta 2.19\left(3 \mathrm{H}, \mathrm{s}, \mathrm{N}=\mathrm{C}-\mathrm{CH}_{3}\right)$, $3.38\left(\mathrm{~m}, 1 \mathrm{H}\right.$, pyrazoline- $\left.\mathrm{H}_{4}\right), 4.08\left(\mathrm{dd}, 1 \mathrm{H}, J_{1,2} 13\right.$, $J_{1,3} 18 \mathrm{~Hz}$, pyrazoline- $\left.\mathrm{H}_{4}\right), 6.91\left(\mathrm{dd}, 1 \mathrm{H}, J_{1,2} 9, J_{1,3} 13 \mathrm{~Hz}\right.$, pyrazoline- $\left.\mathrm{H}_{5}\right), 7.22\left(\mathrm{~s}, 2 \mathrm{H}, \mathrm{NH}_{2} ; \mathrm{D}_{2} \mathrm{O}\right.$ exchangeable), 7.37-7.43 (m, 2H, ArH), 7.51-7.57 (m, 4H, ArH), 7.67 (d, $2 \mathrm{H}, J 7.0 \mathrm{~Hz}, \mathrm{ArH}), 7.88$ (d, 2H, J 8.0 Hz, ArH), 8.09 (d, $2 \mathrm{H}, J 8.0 \mathrm{~Hz}, \mathrm{ArH}), 8.57$ (s, $1 \mathrm{H}, \mathrm{H}_{\text {anth-10 }}$ ). Anal. Calc. for $\mathrm{C}_{25} \mathrm{H}_{21} \mathrm{ClN}_{4}$ (412.91): C, 72.72; H, 5.13; N, 13.57. Found: C, 72.63; H, 4.95; N, $13.73 \%$.

5-(Anthracen-9-yl)-7-(4-chlorophenyl)-3-methyl-1phenyl-1, 5-dihydropyrazolo[1,2-a]tetrazole (20)

A mixture of 4,5-dihydro- $1 H$-pyrazole derivative $\mathbf{1 4}$ $(0.49 \mathrm{~g}, 0.001 \mathrm{~mol})$ and acetic anhydride $(10 \mathrm{~mL})$ was heated on a boiling water bath for $5 \mathrm{~h}$. The reaction mixture was poured onto crushed ice, the precipitated product was filtered, washed with water, dried and recrystallized from ethanol/chloroform, furnishing $\mathbf{2 0}$.

Yield: $65 \%$, buff crystals, mp $169-170{ }^{\circ} \mathrm{C} .{ }^{1} \mathrm{H}$ NMR $\left(\right.$ DMSO- $\left.d_{6}\right) \delta 2.32\left(3 \mathrm{H}, \mathrm{s}, \mathrm{CH}_{3}\right), 4.34-4.38(\mathrm{~m}, 1 \mathrm{H}$, pyrazoline- $\left.\mathrm{H}_{4}\right), 4.50-4.53\left(\mathrm{~m}, 1 \mathrm{H}\right.$, pyrazoline- $\left.\mathrm{H}_{5}\right), 7.27$ $(\mathrm{t}, 2 \mathrm{H}, J 7.6 \mathrm{~Hz}, \mathrm{ArH}), 7.37-7.51(\mathrm{~m}, 3 \mathrm{H}, \mathrm{ArH}), 7.68(\mathrm{~d}$, $2 \mathrm{H}, J 8.0 \mathrm{~Hz}, \mathrm{ArH}), 7.90-7.91(\mathrm{~m}, 2 \mathrm{H}, \mathrm{ArH}), 7.99$ (d, $2 \mathrm{H}, J 8.0 \mathrm{~Hz}, \mathrm{ArH}), 8.04$ (d, 2H, J 7.6 Hz, ArH), 8.12 (d, $2 \mathrm{H}, J 7.6 \mathrm{~Hz}, \mathrm{ArH}), 8.17-8.19$ (m, 2H, ArH), 8.68 (s, 1H, $\mathrm{H}_{\text {anth-10 }}$ ). ${ }^{13} \mathrm{C}$ NMR (DMSO) $\delta 22.16,56.88,122.93,123.11$, 124.36, 126.79, 127.04, 128.07, 128.30, 128.95, 129.11, $129.47,129.69,130.07,130.38,130.51,130.58,131.44$, 131.95, 132.94, 135.62, 154.09. Anal. Calc. for $\mathrm{C}_{31} \mathrm{H}_{23} \mathrm{ClN}_{4}$ (486.99): C, 76.46; H, 4.76; N, 11.50. Found: C, 76.53; H, $4.81 ; \mathrm{N}, 11.41 \%$.
General procedure for the preparation of compounds 21-23

To a solution of 3-(anthracen-9-yl)-1-(aryl)prop-2en-1-one (1, $0.34 \mathrm{~g}, 0.001 \mathrm{~mol}$ or $2,0.32 \mathrm{~g}, 0.001 \mathrm{~mol})$ in absolute ethanol $(20 \mathrm{~mL})$ was added the proper aryl hydrazine hydrochloride $(0.0012 \mathrm{~mol})$. The reaction mixture was heated under reflux for $4 \mathrm{~h}$. A solid product was obtained after concentration and cooling. The precipitated product was filtered, washed with ethanol, dried, and recrystallized from ethanol/chloroform.

5-(Anthracen-9-yl)-1-(4-bromophenyl)-3-(4chlorophenyl)-4, 5-dihydro-1H-pyrazole (21)

Yield: $70 \%$, buff crystals, mp $299-300{ }^{\circ} \mathrm{C} .{ }^{1} \mathrm{H}$ NMR $\left(\right.$ DMSO- $\left.d_{6}\right) \delta 3.12-3.39\left(1 \mathrm{H}, \mathrm{m}\right.$, pyrazoline- $\left.\mathrm{H}_{4}\right), 3.94-4.06$ $\left(1 \mathrm{H}, \mathrm{m}\right.$, pyrazoline- $\left.\mathrm{H}_{4}\right), 6.86-6.94\left(1 \mathrm{H}, \mathrm{m}\right.$, pyrazoline- $\left.\mathrm{H}_{5}\right)$, 7.03-7.15 (m, 4H, ArH), 7.30 (d, 2H, J 7.6 Hz, ArH), 7.41 (t, $2 \mathrm{H}, J 7.6 \mathrm{~Hz}, \mathrm{ArH}), 7.48$ (t, 2H, $J 7 \mathrm{~Hz}, \mathrm{Ar}-\mathrm{H}), 7.71$ (d, 2H, J $8.0 \mathrm{~Hz}, \mathrm{ArH}), 7.91$ (d, 2H, J $8.0 \mathrm{~Hz}, \mathrm{ArH}), 8.05$ (d, $2 \mathrm{H}, J 8.0 \mathrm{~Hz}, \mathrm{ArH}), 8.53$ (s, 1H, $\mathrm{H}_{\text {anth-10 }}$ ). Anal. Calc. for $\mathrm{C}_{29} \mathrm{H}_{20} \mathrm{BrClN}_{2}$ (511.84): C, 68.05; H, 3.94; N, 5.47. Found: C, 68.27; H, 4.09; N, 5.22\%.

5-(Anthracen-9-yl)-1-(4-bromophenyl)-3-p-tolyl)-4,5dihydro-1H-pyrazole (22)

Yield: $73 \%$, brown crystals, mp $130-131{ }^{\circ} \mathrm{C} .{ }^{1} \mathrm{H}$ NMR $\left(\mathrm{DMSO}-d_{6}\right) \delta 2.17\left(\mathrm{~s}, 3 \mathrm{H}, p-\mathrm{CH}_{3}\right), 3.34(\mathrm{dd}, 1 \mathrm{H}$, $J_{1,2} 9, J_{1,3} 18 \mathrm{~Hz}$, pyrazoline- $\left.\mathrm{H}_{4}\right), 4.09\left(\mathrm{dd}, 1 \mathrm{H}, J_{1,2} 13\right.$, $J_{l, 3} 18 \mathrm{~Hz}$, pyrazoline- $\left.\mathrm{H}_{4}\right), 6.86\left(\mathrm{dd}, 1 \mathrm{H}, J_{1,2} 9, J_{1,3} 13 \mathrm{~Hz}\right.$, pyrazoline- $\left.\mathrm{H}_{5}\right), 7.34(\mathrm{t}, 2 \mathrm{H}, J 7.6 \mathrm{~Hz}, \mathrm{ArH}), 7.41(\mathrm{t}, 2 \mathrm{H}, J 7.6$ $\mathrm{Hz}, \mathrm{ArH}$ ), 7.49-7.58 (m, 5H, Ar-H), 7.62 (d, 2H, J 9.0 Hz, ArH), 7.85 (d, 2H, J $8.0 \mathrm{~Hz}, \mathrm{ArH}), 8.08$ (d, 2H, J $8.0 \mathrm{~Hz}$, $\mathrm{ArH})$, 8.54-8.56 (m, 1H, ArH). Anal. Calc. for $\mathrm{C}_{30} \mathrm{H}_{23} \mathrm{BrN}_{2}$ (491.42): C, 73.32; H, 4.72; N, 5.70. Found: C, 73.59; H, $4.61 ; \mathrm{N}, 5.51 \%$.

5-(Anthracen-9-yl)-1-(naphthalene-2-yl)-3-p-tolyl-4, 5-dihydro-1H-pyrazole (23)

Yield: $77 \%$, pale brown crystals, mp $160-161{ }^{\circ} \mathrm{C}$. ${ }^{1} \mathrm{H}$ NMR (DMSO- $d_{6}$ ) $\delta 2.44$ (3H, s, $p-\mathrm{CH}_{3}$ ), 2.99-3.39 (m, $1 \mathrm{H}$, pyrazoline- $\left.\mathrm{H}_{4}\right), 3.78-4.04\left(\mathrm{~m}, 1 \mathrm{H}\right.$, pyrazoline- $\left.\mathrm{H}_{4}\right)$, 6.92-6.98 (m, 1H, pyrazoline- $\left.\mathrm{H}_{5}\right), 7.21-7.27(\mathrm{~m}, 4 \mathrm{H}$, ArH ), 7.29 (dd, 2H, J 7.6, $13 \mathrm{~Hz}, \mathrm{ArH}), 7.39-7.43$ (m, 2H, ArH), 7.57-7.79 (m, 4H, ArH), 7.81 (dd, 1H, J 3.0, $5.0 \mathrm{~Hz}, \mathrm{ArH}), 7.97$ (dd, 2H, J 7.6, $13 \mathrm{~Hz}$ ), 8.20-8.28 (m, $2 \mathrm{H}, \mathrm{ArH}), 8.31$ (dd, 1H, J 3.0, 5.0 Hz, ArH), 8.50 (s, $1 \mathrm{H}, \mathrm{ArH}), 8.52$ (s, 1H, ArH). Anal. Calc. for $\mathrm{C}_{34} \mathrm{H}_{26} \mathrm{~N}_{2}$ (462.58): C, 88.28; H, 5.57; N, 6.06. Found: C, 88.05; $\mathrm{H}, 5.43 ; \mathrm{N}, 6.24 \%$. 
General procedure for the preparation of compounds $\mathbf{2 4}$ and 25

A mixture of 3-(anthracen-9-yl)-1-(aryl)prop-2-en1-one (1, $0.34 \mathrm{~g}, 0.001 \mathrm{~mol}$ or $2,0.32 \mathrm{~g}, 0.001 \mathrm{~mol})$ and hydrazine hydrate $(3 \mathrm{~mL})$ in ethanol $(20 \mathrm{~mL})$ was heated under reflux for $4 \mathrm{~h}$. The solid product was obtained after concentration and cooling, the precipitated product was filtered, washed with ethanol, dried and recrystallized from ethanol.

5-(Anthracen-9-yl)-3-(4-chlorophenyl)-4, 5-dihydro- $1 \mathrm{H}$ pyrazole (24)

Yield: $74 \%$, pale brown crystals, mp $230-231{ }^{\circ} \mathrm{C}$. IR (KBr) $v_{\max } / \mathrm{cm}^{-1}: 3254(\mathrm{NH}) ;{ }^{1} \mathrm{H}$ NMR (DMSO- $d_{6}$ ) $\delta$ 3.60-4.11 (m, 2H, pyrazoline $\left.-\mathrm{H}_{4}, \mathrm{H}_{4}\right), 6.71-6.74(\mathrm{~m}, 1 \mathrm{H}$, pyrazoline- $\left.\mathrm{H}_{5}\right), 7.01-7.11(\mathrm{~m}, 2 \mathrm{H}, \mathrm{ArH}), 7.25-7.40(\mathrm{~m}, 2 \mathrm{H}$, $\mathrm{ArH})$, 7.50-7.71 (m, 4H, ArH), 7.83-7.91 (m, 4H, ArH), 8.18 (s, $\left.1 \mathrm{H}, \mathrm{H}_{\text {anth-10 }}\right), 8.56$ (s, 1H, $\mathrm{NH} ; \mathrm{D}_{2} \mathrm{O}$ exchangeable), MS m/z: 356, $358(\mathrm{M})$. Anal. Calc. for $\mathrm{C}_{23} \mathrm{H}_{17} \mathrm{ClN}_{2}$ (356.85): C, 77.41; H, 4.80; N, 7.85. Found: C, 77.60; H, 4.60; N, $7.63 \%$.

5-(Anthracen-9-yl)-3-p-tolyl-4, 5-dihydro-1H-pyrazole (25)

Yield: $71 \%$, reddish brown crystals, mp $145-146^{\circ} \mathrm{C}$. IR $(\mathrm{KBr}) v_{\max } / \mathrm{cm}^{-1}: 3284(\mathrm{NH}) ;{ }^{1} \mathrm{H}$ NMR (DMSO- $\left.d_{6}\right) \delta 2.44$ (s, $\left.3 \mathrm{H}, p-\mathrm{CH}_{3}\right), 3.42-4.08\left(\mathrm{~m}, 2 \mathrm{H}\right.$, pyrazoline- $\left.\mathrm{H}_{4}, \mathrm{H}_{4}\right)$, 6.60-6.73 (m, 1H, pyrazoline- $\left.\mathrm{H}_{5}\right), 7.17(\mathrm{~d}, 2 \mathrm{H}, J 8.0 \mathrm{~Hz}$, $\mathrm{ArH}), 7.64(\mathrm{~d}, 2 \mathrm{H}, J 7.6 \mathrm{~Hz}, \mathrm{ArH}), 7.70(\mathrm{~d}, 2 \mathrm{H}, J 7.6 \mathrm{~Hz}$, ArH), 7.90 (dd, 2H, J 3.0, $5.0 \mathrm{~Hz}, \mathrm{ArH}), 8.12$ (d, 2H, J 8.0 $\mathrm{Hz}, \mathrm{ArH}), 8.18$ (dd, 2H, J 3.0, 5.0 Hz, ArH), 8.47 (s, 1H, $\mathrm{H}_{\text {anth-10 }}$ ), 9.63 (s, $1 \mathrm{H}, \mathrm{NH} ; \mathrm{D}_{2} \mathrm{O}$ exchangeable). Anal. Calc. for $\mathrm{C}_{24} \mathrm{H}_{20} \mathrm{~N}_{2}$ (336.43): C, 85.68; H, 5.99; N, 8.33. Found: C, 85.60; H, 5.79; N, 8.52\%.

General procedure for the preparation of compounds $\mathbf{2 6}$ and 27

To an ice cold solution of 5-(anthracen-9-yl)-3-aryl-4,5dihydro- $1 H$-pyrazole 24 ( $0.35 \mathrm{~g}, 0.001 \mathrm{~mol})$ or $\mathbf{2 5}(0.33 \mathrm{~g}$, $0.001 \mathrm{~mol})$ in hydrochloric acid $(50 \%, 15 \mathrm{~mL})$ was added $\mathrm{NaNO}_{2}(0.07 \mathrm{~g}, 0.001 \mathrm{~mol})$ and the reaction mixture was stirred for $3 \mathrm{~h}$. The separated solid product was filtered, washed with ethanol, dried and recrystallized from ethanol.

5-(Anthracen-9-yl)-3-(4-chlorophenyl)-1-nitroso-4,5dihydro-1H-pyrazole (26)

Yield: $89 \%$, pale brown crystals, mp $150-151^{\circ} \mathrm{C}$. ${ }^{1} \mathrm{H}$ NMR (DMSO- $d_{6}$ ) $\delta 3.28\left(\mathrm{dd}, 1 \mathrm{H}, J_{1,2} 13.8, J_{1,3} 16.8 \mathrm{~Hz}\right.$, pyrazoline- $\left.\mathrm{H}_{4}\right), 3.67\left(\mathrm{dd}, 1 \mathrm{H}, J_{l, 2} 13.8, J_{1,3} 16.8 \mathrm{~Hz}\right.$, pyrazoline- $\left.\mathrm{H}_{4}\right), 6.39\left(\mathrm{t}, 1 \mathrm{H}, J 13.8 \mathrm{~Hz}\right.$, pyrazoline- $\left.\mathrm{H}_{5}\right), 7.43-$ $7.52(\mathrm{~m}, 6 \mathrm{H}, \mathrm{ArH}), 7.69$ (d, 2H, J 8.0 Hz, ArH), 8.06 (d, 2H, $J 7.6 \mathrm{~Hz}, \mathrm{ArH}), 8.41$ (d, 2H, J 8.0 Hz, ArH), 8.55 (s, 1H, $\left.\mathrm{H}_{\text {anth-10 }}\right)$. Anal. Calc. for $\mathrm{C}_{23} \mathrm{H}_{16} \mathrm{ClN}_{3} \mathrm{O}$ (385.85): C, 71.59; H, 4.18; N, 10.89. Found: C, 71.70; H, 4.32; N, 10.70\%.

5-(Anthracen-9-yl)-1-nitroso3-p-tolyl-4,5-dihydro-1Hpyrazole (27)

Yield: $84 \%$, buff crystals, mp $129-130{ }^{\circ} \mathrm{C} .{ }^{1} \mathrm{H}$ NMR $\left(\right.$ DMSO- $\left.d_{6}\right) \delta 2.43\left(3 \mathrm{H}, \mathrm{s}, p-\mathrm{CH}_{3}\right), 3.25-3.44(\mathrm{~m}, 1 \mathrm{H}$, pyrazoline- $\left.\mathrm{H}_{4}\right), 3.69-4.09\left(\mathrm{~m}, 1 \mathrm{H}\right.$, pyrazoline- $\left.\mathrm{H}_{4}\right), 7.00-$ $7.24\left(\mathrm{~m}, 1 \mathrm{H}\right.$, pyrazoline- $\left.\mathrm{H}_{5}\right), 7.18(\mathrm{~d}, 2 \mathrm{H}, J 9 \mathrm{~Hz}, \mathrm{ArH})$, 7.39 (d, 2H, J 9.0 Hz, ArH), 7.59 (t, $2 \mathrm{H}, J 8.0 \mathrm{~Hz}, \mathrm{ArH})$, 7.69 (d, 2H, J $8.0 \mathrm{~Hz}, \mathrm{ArH}), 7.82$ (t, 2H, J $8 \mathrm{~Hz}, \mathrm{ArH}), 7.88$ (d, $2 \mathrm{H}, J 8.0 \mathrm{~Hz}, \mathrm{ArH}), 8.17$ (s, 1H, $\mathrm{H}_{\text {anth-10 }}$ ). Anal. Calc. for $\mathrm{C}_{24} \mathrm{H}_{19} \mathrm{~N}_{3} \mathrm{O}$ (365.43): C, 78.88; H, 5.24; $\mathrm{N}, 11.50$. Found: C, 78.75; H, 4.98; N, 11.74\%.

General procedure for the preparation of compounds $\mathbf{2 8}$ and 29

To a solution of 5-(anthracen-9-yl)-3-aryl-4,5dihydro- $1 \mathrm{H}$-pyrazole $(\mathbf{2 4}, 0.35 \mathrm{~g}, 0.001 \mathrm{~mol}$ or $\mathbf{2 5}, 0.33 \mathrm{~g}$, $0.001 \mathrm{~mol})$ in dry ether $(20 \mathrm{~mL})$ was added an equal amount of phenylisothiocyanate $(0.14 \mathrm{~g})$ and the reaction mixture was stirred for $6 \mathrm{~h}$. The solid product obtained was filtered, washed with ethanol, dried and recrystallized from ethanol.

5-(Anthracen-9-yl)-3-(4-chlorophenyl)-N-phenyl-4,5dihydro-1H-pyrazole-1-carbothioamide (28)

Yield: $82 \%$, pale grey crystals, mp $250-251^{\circ} \mathrm{C}$. IR (KBr) $v_{\max } / \mathrm{cm}^{-1}: 949,1143,1346,1552$ (NCS amide I, II, III, IV respectively), $3260(\mathrm{NH}) ;{ }^{1} \mathrm{H}$ NMR (DMSO- $d_{6}$ ) $\delta 3.29-3.31$ $\left(\mathrm{m}, 1 \mathrm{H}\right.$, pyrazoline- $\left.\mathrm{H}_{4}\right), 3.82-3.95\left(\mathrm{~m}, 1 \mathrm{H}\right.$, pyrazoline- $\left.\mathrm{H}_{4}\right)$, 7.10-7.25 (m, 1H, pyrazoline- $\left.\mathrm{H}_{5}\right), 7.30-7.35(\mathrm{~m}, 2 \mathrm{H}, \mathrm{ArH})$, 7.39-7.45 (m, 3H, ArH), 7.48-7.54 (m, 2H, ArH), 7.58-7.64 (m, 2H, ArH), 7.69-7.73 (d, 2H, J 8.0 Hz, ArH), 7.78-7.86 (d, 2H, J 7.0 Hz, ArH), 7.90-7.97 (m, 2H, ArH), 8.08-8.17 (m, 2H, ArH), $8.17\left(\mathrm{~s}, 1 \mathrm{H}, \mathrm{H}_{\text {anth-10 }}\right), 11.10\left(\mathrm{~s}, 1 \mathrm{H}, \mathrm{NH} ; \mathrm{D}_{2} \mathrm{O}\right.$ exchangeable). Anal. Calc. for $\mathrm{C}_{30} \mathrm{H}_{22} \mathrm{ClN}_{3} \mathrm{~S}$ (492.03): C, $73.23 ; \mathrm{H}, 4.51 ; \mathrm{N}, 8.54$. Found: C, 73.40; H, 4.62; N, 8.30\%.

5-(Anthracen-9-yl)-N-phenyl-3-p-tolyl-4,5-dihydro- $1 \mathrm{H}$ pyrazole-1-carbothioamide (29)

Yield: $85 \%$, buff crystals, mp $215-216^{\circ} \mathrm{C}$. IR $(\mathrm{KBr})$ $v_{\max } / \mathrm{cm}^{-1}: 938,1168,1327,1588$ (NCS amide I, II, III, IV respectively), $3243(\mathrm{NH}) ;{ }^{1} \mathrm{H}$ NMR (DMSO- $\left.d_{6}\right) \delta 2.44$ (s, $\left.3 \mathrm{H}, p-\mathrm{CH}_{3}\right), 3.34-3.69\left(\mathrm{~m}, 1 \mathrm{H}\right.$, pyrazoline- $\left.\mathrm{H}_{4}\right), 3.80-4.03$ $\left(\mathrm{m}, 1 \mathrm{H}\right.$, pyrazoline- $\left.\mathrm{H}_{4}\right), 7.28-7.33(\mathrm{~m}, 2 \mathrm{H}, \mathrm{ArH}), 7.36-7.41$ (m, 2H, ArH), 7.44-7.49 (m, 3H, ArH), 7.51-7.58 (m, 1H, 
pyrazoline- $\left.\mathrm{H}_{5}\right), 7.62-7.68(\mathrm{~d}, 2 \mathrm{H}, J 9 \mathrm{~Hz}, \mathrm{ArH})$ 7.70-7.75 (m, 2H, ArH), 7.79-7.83 (d, 2H, J 8.0 Hz, ArH), 7.86-7.90 (m, 2H, ArH), 8.04-8.10 (m, 2H, ArH), 8.39 (s, 1H, $\mathrm{H}_{\text {anth-10 }}$ ), $11.15\left(\mathrm{~s}, 1 \mathrm{H}, \mathrm{NH} ; \mathrm{D}_{2} \mathrm{O}\right.$ exchangeable). Anal. Calc. for $\mathrm{C}_{31} \mathrm{H}_{25} \mathrm{~N}_{3} \mathrm{~S}$ (471.62): C, 78.95; H, 5.34; N, 8.91. Found: C, 78.76; H, 5.12; N, 9.00\%.

General procedure for the preparation of compounds $\mathbf{3 0}$ and 31

To a solution of 5-(anthracen-9-yl)-3-aryl-4, 5-dihydro$1 H$-pyrazole 24 ( $0.35 \mathrm{~g}, 0.001 \mathrm{~mol})$ or 25 ( $0.33 \mathrm{~g}, 0.001 \mathrm{~mol})$ in dry pyridine $(10 \mathrm{~mL})$ was added an equivalent amount of $p$-tosyl chloride $(0.19 \mathrm{~g})$. The reaction mixture was heated on a boiling water bath for $3 \mathrm{~h}$, cooled and then poured onto crushed ice. The solid product separated was filtered, washed with water, dried and recrystallized from ethanol.

5-(Anthracen-9-yl)-3-(4-chlorophenyl)-1-tosyl-4,5dihydro-1H-pyrazole (30)

Yield: $76 \%$, buff crystals, mp 135-136 ${ }^{\circ} \mathrm{C} .{ }^{1} \mathrm{H}$ NMR $\left(\right.$ DMSO- $\left.d_{6}\right) \delta 2.44\left(3 \mathrm{H}, \mathrm{s}, \mathrm{CH}_{3}\right), 3.42-4.02(\mathrm{~m}, 2 \mathrm{H}$, pyrazoline- $\left.\mathrm{H}_{4}, \mathrm{H}_{4}\right)$, 6.60-6.67 (m, $1 \mathrm{H}$, pyrazoline- $\left.\mathrm{H}_{5}\right), 7.35$ (d, 2H, J 7.6 Hz, ArH), 7.43 (d, 2H, J 8 Hz, ArH), 7.56 (t, $2 \mathrm{H}, J 8.0 \mathrm{~Hz}, \mathrm{ArH}), 7.76$ (d, 2H, J $8.0 \mathrm{~Hz}, \mathrm{ArH}), 7.91$ (t, 2H, $J 8.0 \mathrm{~Hz}, \mathrm{ArH}), 8.01(\mathrm{~m}, 2 \mathrm{H}, \mathrm{ArH}), 8.13(\mathrm{~d}, 2 \mathrm{H}, J 7.6 \mathrm{~Hz}$, ArH), 8.51 (m, 2H, ArH), 8.57 (s, 1H, $\left.\mathrm{H}_{\text {anth-10 }}\right)$. Anal. Calc. for $\mathrm{C}_{30} \mathrm{H}_{23} \mathrm{ClN}_{2} \mathrm{O}_{2} \mathrm{~S}$ (511.03): C, 70.51; H, 4.54; N, 5.48. Found: C, 70.32; H, 4.45; N, 5.69\%.

5-(Anthracen-9-yl)-3-p-tolyl-1-tosyl-4,5-dihydro- $1 \mathrm{H}$ pyrazole (31)

Yield: $74 \%$, buff crystals, mp 200-201 ${ }^{\circ} \mathrm{C} .{ }^{1} \mathrm{H}$ NMR $\left(\right.$ DMSO- $\left.d_{6}\right) \delta 2.41\left(\mathrm{~s}, 3 \mathrm{H}, \mathrm{CH}_{3}\right), 2.44\left(\mathrm{~s}, 3 \mathrm{H}, \mathrm{CH}_{3}\right), 3.40-4.00$ (m, $2 \mathrm{H}$, pyrazoline- $\left.\mathrm{H}_{4}, \mathrm{H}_{4}\right)$, 6.60-6.64 $\left(\mathrm{m}, 1 \mathrm{H}\right.$, pyrazoline- $\left.\mathrm{H}_{5}\right)$, 7.05 (d, 2H, J $3 \mathrm{~Hz}, \mathrm{ArH}), 7.22$ (d, 2H, J $8 \mathrm{~Hz}, \mathrm{ArH}), 7.29$ (t, 2H, J $8 \mathrm{~Hz}, \mathrm{ArH}), 7.33$ (d, 2H, J 7.0 Hz, ArH), 7.38 (d, 2H, J 15.0 Hz, ArH), 7.67 (d, 2H, J 7.0 Hz, ArH), 7.89 (d, $2 \mathrm{H}, J 15.0 \mathrm{~Hz}, \mathrm{ArH}), 7.90$ (t, 2H, J3.0 Hz ArH), 8.47 (s, 1H, $\mathrm{H}_{\text {anth-10 }}$ ). Anal. Calc. for $\mathrm{C}_{31} \mathrm{H}_{26} \mathrm{~N}_{2} \mathrm{O}_{2} \mathrm{~S}$ (490.62): C, 75.89; H, 5.34; N, 5.71. Found: C, 75.70; H, 5.22; N, 5.89\%.

\section{General procedure for the preparation of compounds 32-34}

A mixture of 3-(anthracen-9-yl)-1-(aryl)prop-2-en1-one 1-3 (0.01 mol) and guanidine carbonate $(1.21 \mathrm{~g}$, $0.01 \mathrm{~mol})$ in ethanol $(50 \mathrm{~mL})$ was heated under reflux, while a $5 \mathrm{~mol} \mathrm{~L}^{-1}$ solution of sodium hydroxide $(10 \mathrm{~mL})$ was added portion-wise during one hour. Refluxing was continued for a further $10 \mathrm{~h}$, when the reaction mixture was concentrated, diluted with water and extracted with benzene. The solid product was obtained after concentration and cooling; the precipitated product was filtered, dried and recrystallized from chloroform.

\section{4-(Anthracen-9-yl)-6-(4-chlorophenyl)pyrimidin-2-amine} (32)

Yield: $83 \%$, buff crystals, mp $310-311{ }^{\circ} \mathrm{C}$. IR (KBr) $v_{\max } / \mathrm{cm}^{-1}: 3054,3358\left(\mathrm{NH}_{2}\right) ;{ }^{1} \mathrm{H}$ NMR (DMSO- $\left.d_{6}\right) \delta 5.97$ (s, $2 \mathrm{H}, \mathrm{NH}_{2} ; \mathrm{D}_{2} \mathrm{O}$ exchangeable), $7.17(\mathrm{~d}, 2 \mathrm{H}, J 8.0 \mathrm{~Hz}$, $\mathrm{ArH}$;), 7.35 (s, 1H, pyrimidine- $\left.\mathrm{H}_{5}\right), 7.44(\mathrm{t}, 2 \mathrm{H}, J 8.0 \mathrm{~Hz}$, ArH), 7.59 (t, 2H, $J 8 \mathrm{~Hz}, \mathrm{ArH}), 7.98(\mathrm{~d}, 2 \mathrm{H}, J 8.0 \mathrm{~Hz}$, ArH), 8.05 (d, 2H, J 9.0 Hz, ArH), 8.18 (d, 2H, J $9.0 \mathrm{~Hz}$, ArH), 8.68 (s, $1 \mathrm{H}, \mathrm{H}_{\text {anth-10 }}$ ). Anal. Calc. for $\mathrm{C}_{24} \mathrm{H}_{16} \mathrm{ClN}_{3}$ (381.86): C, 75.49; H, 4.22; N, 11.00. Found: C, 75.21; $\mathrm{H}, 4.00 ; \mathrm{N}, 11.26 \%$.

\section{4-(Anthracen-9-yl)-6-p-tolylpyrimidin-2-amine (33)}

Yield: $81 \%$, buff crystals, mp $210-211^{\circ} \mathrm{C}$. IR $(\mathrm{KBr})$ $v_{\max } / \mathrm{cm}^{-1}: 3142,3366\left(\mathrm{NH}_{2}\right) ;{ }^{1} \mathrm{H}$ NMR (DMSO- $\left.d_{6}\right) \delta 2.25$ (s, $\left.3 \mathrm{H}, p-\mathrm{CH}_{3}\right), 6.07$ (s, 2H, $\mathrm{NH}_{2} ; \mathrm{D}_{2} \mathrm{O}$ exchangeable), 7.46$7.52(\mathrm{~m}, 4 \mathrm{H}, \mathrm{ArH}), 7.57-7.59$ (m, 4H, ArH), 7.63-7.65 (d, $2 \mathrm{H}, J 9.0 \mathrm{~Hz}, \mathrm{ArH}), 7.71-7.86$ (d, 2H, J 8.0 Hz, ArH), 7.91 (s, $1 \mathrm{H}$, pyrimidine- $\left.\mathrm{H}_{5}\right), 8.18\left(\mathrm{~s}, 1 \mathrm{H}, \mathrm{H}_{\text {anth-10 }}\right)$. Anal. Calc. for $\mathrm{C}_{25} \mathrm{H}_{19} \mathrm{~N}_{3}$ (361.44): C, 83.08; H, 5.30; N, 11.63. Found: $\mathrm{C}$, 83.29; H, 5.56; N, $11.42 \%$.

4-(Anthracen-9-yl)-6-(furan-2-yl)pyrimidin-2-amine (34)

Yield:78\%, buff crystals, mp 263-264 ${ }^{\circ} \mathrm{C}$. IR (KBr) $v_{\max } / \mathrm{cm}^{-1}: 3147,3323\left(\mathrm{NH}_{2}\right) ;{ }^{1} \mathrm{H}$ NMR (DMSO- $\left.d_{6}\right)$ $\delta 6.26\left(\mathrm{~s}, 2 \mathrm{H}, \mathrm{NH}_{2} ; \mathrm{D}_{2} \mathrm{O}\right.$ exchangeable), $7.53(\mathrm{~s}, 1 \mathrm{H}$, pyrimidine- $\left.\mathrm{H}_{5}\right), 7.60-7.64(\mathrm{~m}, 1 \mathrm{H}, \mathrm{ArH}), 7.73-7.80(\mathrm{~m}$, $1 \mathrm{H}, \mathrm{ArH}), 7.85-7.89$ (m, 1H, ArH), 7.98 (dd, 2H, J 3.0, $6.0 \mathrm{~Hz}, \mathrm{ArH}), 8.06-8.08$ (m, 4H, ArH), 8.17 (dd, 2H, J3.0, $6.0 \mathrm{~Hz}, \mathrm{ArH}), 8.33$ (s, $1 \mathrm{H}, \mathrm{H}_{\text {anth-10 }}$ ). Anal. Calc. for $\mathrm{C}_{22} \mathrm{H}_{15} \mathrm{~N}_{3} \mathrm{O}$ (337.37): C, 78.32; H, 4.48; N, 12.46. Found: C, $78.56 ; \mathrm{H}, 4.27 ; \mathrm{N}, 12.25 \%$.

General procedure for the preparation of compounds $\mathbf{3 5}$ and $\mathbf{3 6}$

A mixture of 4-(anthracen-9-yl)-6-arylpyrimidin-2amine (32, $0.38 \mathrm{~g}, 0.001 \mathrm{~mol}$ or 33, $0.36 \mathrm{~g}, 0.001 \mathrm{~mol})$ and acetic anhydride $(10 \mathrm{~mL})$ was heated on boiling water bath for $2 \mathrm{~h}$. The reaction mixture was poured onto cold water and the precipitated product was filtered, washed with water, dried and recrystallized from ethanol/chloroform.

$N$-(4-(Anthracen-9-yl)-6-(4-chlorophenyl)pyrimidin-2-yl) acetamide (35)

Yield: $83 \%$, buff crystals, mp $130-131{ }^{\circ} \mathrm{C}$. IR $(\mathrm{KBr})$ $v_{\max } / \mathrm{cm}^{-1}: 1667(\mathrm{C}=\mathrm{O}), 3260(\mathrm{NH}) ;{ }^{1} \mathrm{H}$ NMR (DMSO- $d_{6}$ ) 
$\delta 2.25$ (s, 3H, $\left.\mathrm{NCOCH}_{3}\right), 7.28$ (t, $\left.2 \mathrm{H}, J 8.0 \mathrm{~Hz}, \mathrm{ArH}\right), 7.47$ (t, 2H, J 6.0 Hz, ArH), 7.61 (d, 2H, J 7.6 Hz, ArH), 7.89 (d, $2 \mathrm{H}, J 6.0 \mathrm{~Hz}, \mathrm{ArH}), 8.00$ (s, $1 \mathrm{H}$, pyrimidine- $\left.\mathrm{H}_{5}\right), 8.06$ (d, $2 \mathrm{H}, J 8.0 \mathrm{~Hz}, \mathrm{ArH}), 8.17$ (d, 2H, J 7.6 Hz, ArH), 8.70 (s, $\left.1 \mathrm{H}, \mathrm{H}_{\text {anth-10 }}\right), 10.77$ (s, 1H, NH; $\mathrm{D}_{2} \mathrm{O}$ exchangeable). Anal. Calc. for $\mathrm{C}_{26} \mathrm{H}_{18} \mathrm{ClN}_{3} \mathrm{O}$ (423.89): C, 73.67; H, 4.28; N, 9.91. Found: C, 73.89; H, 4.01; N, 9.75\%.

N-(4-(Anthracen-9-yl)-6-p-tolylpyrimidin-2-yl)acetamide (36)

Yield: $78 \%$, buff crystals, mp $119-120{ }^{\circ} \mathrm{C}$. IR (KBr) $v_{\max } / \mathrm{cm}^{-1}: 1669(\mathrm{C}=\mathrm{O}), 3218(\mathrm{NH}) ;{ }^{1} \mathrm{H}$ NMR (DMSO- $\left.d_{6}\right)$ $\delta 2.29$ (s, 3H, $\mathrm{NCOCH}_{3}$ ), 2.36 (s, 3H, $\left.p-\mathrm{CH}_{3}\right), 7.30-7.63$ (m, 4H, ArH), 7.90-7.92 (m, 4H, ArH), 8.17-8.19 (m, 4H, $\mathrm{ArH}), 8.37\left(\mathrm{~s}, 1 \mathrm{H}\right.$, pyrimidine- $\left.\mathrm{H}_{5}\right), 8.74\left(\mathrm{~s}, 1 \mathrm{H}, \mathrm{H}_{\text {anth-10 }}\right)$, 10.47 (s, $1 \mathrm{H}, \mathrm{NH} ; \mathrm{D}_{2} \mathrm{O}$ exchangeable). Anal. Calc. for $\mathrm{C}_{27} \mathrm{H}_{21} \mathrm{~N}_{3} \mathrm{O}$ (403.48): C, 80.37; H, 5.25; N, 10.41. Found: C, $80.56 ; \mathrm{H}, 5.01 ; \mathrm{N}, 10.23 \%$.

\section{Biological activity assay}

\section{Measurement of the inhibition zone (IZ)}

Compounds 1-36 were evaluated in vitro for antimicrobial activity against Escherichia coli ATCC8739 and Pseudomonas aeruginosa ATCC 9027 as gramnegative bacteria, Staphylococcus aureus ATCC 6538P as an example of gram-positive bacteria and Candida albicans ATCC 2091 as yeast-like fungus. The agar-diffusion method ${ }^{33}$ was used for the determination of antibacterial and antifungal activity. From $1 \mathrm{mg} \mathrm{mL}^{-1}$ solutions of each of the test compounds in $N, N$-dimethylformamide (DMF), $75 \mu \mathrm{L}$ was placed in a $6 \mathrm{~mm}$ diameter well in an agar plate seeded with the appropriate test organism in triplicates. Ampicillin trihydrate $(10 \mu \mathrm{g}$ per disc), Ciprofloxacin (5 $\mu \mathrm{g}$ per disc), Imipenem $(10 \mu \mathrm{g}$ per disc) and Clotrimazole $(100 \mu \mathrm{g}$ per disc) were used as standard antibacterial and antifungal agents, respectively. The plates were incubated at $37^{\circ} \mathrm{C}$ for $24 \mathrm{~h}$. The results were recorded for each tested compounds as the average diameter of inhibition zone of bacterial growth in mm (Table 1). DMF alone (control) showed no inhibition zone.

\section{Minimal inhibitory concentration (MIC)}

The microdilution susceptibility test in Muller-Hinton broth (oxoid) and Sabouraud liquid medium (oxoid) were used for the determination of antibacterial and antifungal activity with the same test organisms. The MIC measurements ${ }^{34}$ were carried out for compounds that showed significant inhibition zones using the two-fold serial dilution technique with solutions in the concentration range 500-15.63 $\mu \mathrm{g} \mathrm{mL}^{-1}$. Suspensions of the microorganisms at $10^{6} \mathrm{CFU} \mathrm{mL}^{-1}$ (Colony Forming Units $\mathrm{mL}^{-1}$ ) were used to inoculate the prepared test compounds in the above mentioned serial dilution broth. The culture tubes were incubated at $37^{\circ} \mathrm{C}$ for $24-48 \mathrm{~h}$. At the end of the incubation period the growth of bacteria was observed in the form of turbidity. The MIC is defined as the lowest concentration that showed no bacterial growth (Table 1).

\section{Supplementary Information}

Supplementary data are available free of charge at http://jbcs.sbq.org.br as PDF file.

\section{References}

1. Szliszka, E.; Czuba, Z. C.; Mazur, B. M.; Sedek, L.; Paradysz, A.; Krol, W.; Int. J. Mol. Sci. 2010, 11, 1.

2. Mokle, S. S.; Sayeed, M. A.; Kothawar, C.; Int. J. Chem. Sci. 2004, 2, 96; Rajendra, P. Y.; Lakshmana Rao, A.; Rambabu, R.; E-J. Chem. 2008, 5, 461; Bhasker, N.; Krishna, B.; Raghava, K. R.; Komal, M. R.; Rasayan J. Chem. 2009, 2, 139; Zangade, S. B.; Jadhav, J. D.; Lalpod; Vibhute, Y. B.; Dawane, B. S.; J. Chem. Pharm. Res. 2010, 2, 310.

3. Rojas, J.; Payá, M.; Domínguez, J. N.; Ferrándiz, M. L.; Bioorg. Med. Chem. Lett. 2002, 12, 1951.

4. Echeverría, C.; Santibañez, J. S.; Donoso-Tauda, O.; Escobar, C. A.; Ramírez-Tagle, R.; Int. J. Mol. Sci. 2009, 10, 221; Nowakowska, Z. A.; Eur. J. Med. Chem. 2007, 42, 125; Shah, A.; Khan, A. M.; Qureshi, R.; Ansari, F. L.; Nazar, M. F.; Shah, S. S.; Int. J. Mol. Sci. 2008, 9, 1424; Boumendjel, A.; Ronot, X. B.; Curr. Drug Targets 2009, 10, 363; Katsori, A. M.; Hadjipavlou-Latina, D.; Curr. Med. Chem. 2009, 16, 1062.

5. Viana, G. S.; Bandeira, M. A.; Matos, F.; J. Phytomedicine 2003, 10, 189.

6. Zhao, L. M.; Jin, H. S.; Sun, L. P.; Piao, H. R.; Quan, Z. S.; Bioorg. Med. Chem. Lett. 2005, 15, 5027.

7. Li-Ping, G.; Nan, J.-X.; Jin, X.-J.; Jin, Q.-H.; Kwak, K.-C.; Chai, K.-Y.; Quan, Z.-S.; Arch. Pharm. Res. 2005, 28, 81.

8. Mishra, N.; Arora, P.; Kumar, B.; Mishra L. C.; Bhattacharya, A.; Awasthi, S. K.; Bhasin, V. K.; Eur. J. Med. Chem. 2008, 43, 1530; Valla, A.; Valla, B.; Cartier, D.; Le Guillou, R.; Labia, R.; Florent, L.; Charneau, S.; Schrevel, J.; Potier, P.; Eur. J. Med. Chem. 2006, 41, 142.

9. Jamal, H.; Ansari, W. H.; Rizvi, S. J.; Biol. Med. 2009, 1, 107.

10. Rizvi, S. U. F.; Siddiqui, H. L.; Parvwez, M.; Ahmad, M.; Siddiqui, W. A.; Yasinzai, M. M.; Chem. Pharm. Bull. 2010, 58, 301; Mei, L.; Prapon, W.; Simon, L. C.; Bioorg. Med. Chem. 2003, 11, 2729.

11. Sivakumar, P. M.; Geetha, B. S. K.; Mukesh, D.; Chem. Pharm. Bull. 2007, 55, 44. 
12. Satyanarayana, M.; Priti, T.; Brajendra, K. T.; Srivastava, A. K.; Ram, P.; Bioorg. Med. Chem. 2004, 12, 883.

13. Khatib, S.; Nerya, O.; Musa, R.; Shmuel, M.; Tamir, S.; Vaya, J.; Bioorg. Med. Chem. 2005, 13, 433; Nishida, J.; Gao, H.; Kawabata, J.; Bioorg. Med.Chem. 2007, 15, 2396.

14. Drabu, S.; Rana, A.; Harish, K.; Indian J. Heterocycl. Chem. 2007, 16, 399; Amir, M.; Kumar, S.; Indian J. Chem. 2005, 44B, 2532; Adnan, A. B.; Hayam, M. A. A.; Aida, A. G.; Arch. Pharm. 2005, 338, 167.

15. Verma, B. L.; Singhal, M.; Indian J. Heterocycl. Chem. 2007, 14, 343.

16. Karthikeyan, M. S.; Holla, B. S.; Suchetha, N.; Eur. J. Med. Chem. 2007, 42, 30; Kaplancikli, Z. A.; Turan-Zitouni, G.; Ozdemir, A.; Revial, G.; Guven, K.; Phosphorus, Sulfur, Silicon Relat. Elem. 2007, 182, 749; Ahmet, Ö.; Gülhan, T. Z.; Zafer, A. K.; Gilbert, R.; Kiymet, G.; Eur. J. Med. Chem. 2007, 42, 403; Susant, S. K.; Mrityunjay, B.; Sagar, M. K.; Raj, M. K.; Prasanna, P. K.; Prafulla, M. K.; Acta Pol. Pharm. Drug Res. 2007, 64, 121; Anjani,S.; Sejal, S.; Ghanshayam, P.; Rasayan J. Chem. 2008, 1, 581.

17. Shekarchi, M.; Pirali-Hamedani, B. L.; Navidpour, N.; Adib, Shafiee, A.; J. Iran. Chem. Soc. 2008, 5, 150.

18. Rajendra, P. Y.; Lakshmana, R. A.; Prasoona, L.; Murali, K.; Ravikumar, P.; Bioorg. Med. Chem. Lett. 2005, 15, 5030; Ruhogluo, O.; Ozdemir, Z.; Calis, U.; Gumusel, B.; Bilgin, A. A.; Arzneimittelforschung 2005, 55, 431.

19. Ozdemir, Z.; Kandilici, H. B; Gumusel, B.; Calis, U.; Bilgin, A. A.; Eur. J. Med. Chem. 2007, 42, 373.

20. Brzozwski, Z.; Czewski, F. S.; Gdaniec, M.; Eur. J. Med. Chem. 2000, 35, 1053

21. Harinadha, B. V.; Manna, S. K.; Sneha; Srinivasan, K. K.; Varadaraj, B. G.; Indian J. Heterocycl. Chem. 2004, 13, 253.

22. Kristopher, S. S.; David, M. S.; Pestic. Biochem. Physiol. 2005, 81, 136.

23. Suresh, K.; Sandhya, B.; Sushma, D.; Rajiv, K.; Himanshu, G.; Recent Pat. Anti-Infect. Drug Discovery 2009, 4, 154; Shaharyar, M.; Siddiqui, A. A.; Ali, M. A.; Sriram, D.; Yogeeswari, P.; Bioorg. Med. Chem. Lett. 2006, 16, 3947.

24. Flora, F. B.; Hanaa, M. H.; Adel, S. G.; Bioorg. Med. Chem. 2006, 14, 3929.

25. Shafiee, A.; Bagheri, M.; Shekarchi, M.; Mohammad, A.; J. Pharm. Pharm. Sci. 2003, 6, 360 .
26. Funiss, B. S.; Hannford, A. J.; Smith, P. W. G.; Tatchell, A. R.; Vogel's Textbook of Practical Organic Chemistry, $5^{\text {th }}$ ed., Prentice-Hall: Englewood Cliffs, NJ, 1996, p. 1032; Morrison, R. T.; Boyd, R. N.; Organic Chemistry, $6^{\text {th }}$ ed., Prentice-Hall: Englewood Cliffs, NJ, 1992, p. 971.

27. Revanasiddappa, B. C.; Nagendra, Rao, R.; Subrahmanyam, E. V. S.; Satyanarayana, D.; E-J. Chem. 2010, 7, 295; Jadhav, S. B.; Shastri, R. A.; Gaikwad, K. V.; Gaikwad, S. V.; E-J. Chem. 2009, 6, S183; Ahmet, O. Z. R.; Gülhan, T. Z.; Zafer, A. K.; Turk. J. Chem. 2008, 32, 529; Ragini, G.; Neetu, G.; Anshu, J.; Indian J. Chem. 2010, 49b, 351; Chetana, B. P.; Mahajan, S. K.; Suvarna, A. K.; J. Pharm. Sci. Res. 2009, 3, 11.

28. Chimenti, F.; Bizzarri, B.; Manna, F.; Boalsco, A.; Secci, D.; Chimenti, P.; Granese, A.; Rivanera, D.; Lilli, D.; Scaltrito, M. M.; Brenciaglia, M. I.; Bioorg. Med. Chem. Lett. 2005, 15, 603; Levai, A.; Jeko, J.; Brahmbhatt, D. I.; J. Heterocycl. Chem. 2005, 42, 1231; Levai, A.; J. Heterocycl. Chem. 2005, 39, 1; Levai, A.; Jeko, J.; J. Heterocycl. Chem. 2006, 43, 111; Adnan, A. B.; Tarek, A. A.; Bioorg. Med. Chem. 2004, $12,1935$.

29. Ali, M. A.; Siddiqui, M. S. A. A.; Eur. J. Med. Chem. 2007, 42, 268; Amir, M.; Kumar, H.; Khan, S. A.; Bioorg. Med. Chem. Lett. 2008, 18, 918.

30. Vimesh, M. P.; Kishor, R. D.; Arkivoc 2004, I, 123.

31. Kamal, A.; Laxma Reddy, K.; Devaiah, V.; Shankaraiah, N.; Shiva, M. K.; Suresh, G. K. R.; Lett. Drug Des. Discovery 2005 , 2, 55; Nagaraj, A.; Reddy, S. C.; J. Iran. Chem. Soc. 2008, 5, 262; Vyas, R.; Choudhary, P. C.; Sharma, H.; Verma, B. L.; Indian J. Heterocycl. Chem. 2008, 17, 237; Amir, M.; Javed, S. A.; Acta Pharm. 2008, 58, 467; Arvind, G. M.; Avnish, A. P.; E-J. Chem. 2009, 6, S406.

32. El-Rayyes, N. R.; J. Heterocycl. Chem. 1982, 19, 415.

33. Abou-Elela, G. M.; Ibrahim, H. A.; El-Helow, E.; Sabry, S.; World Appl. Sci. J. 2009, 7, 748.

34. Cruickshank, R.; Duguid, J. P.; Marmion, B. P.; Swam, H. A.; The Practice of Medical Microbiology; $12^{\text {th }}$ ed., Churchill Livingstone, London, 1975.

Submitted: November 21, 2010 Published online: March 11, 2011 


\section{Supplementary Information}

\section{Synthesis and Biological Activity of Some New Pyrazoline and \\ Pyrimidine Derivatives}

\section{Seham Y. Hassan}

Department of Chemistry, Faculty of Science, University of Alexandria,

PO Box 426, 21321 Ibrahimia, Alexandria, Egypt

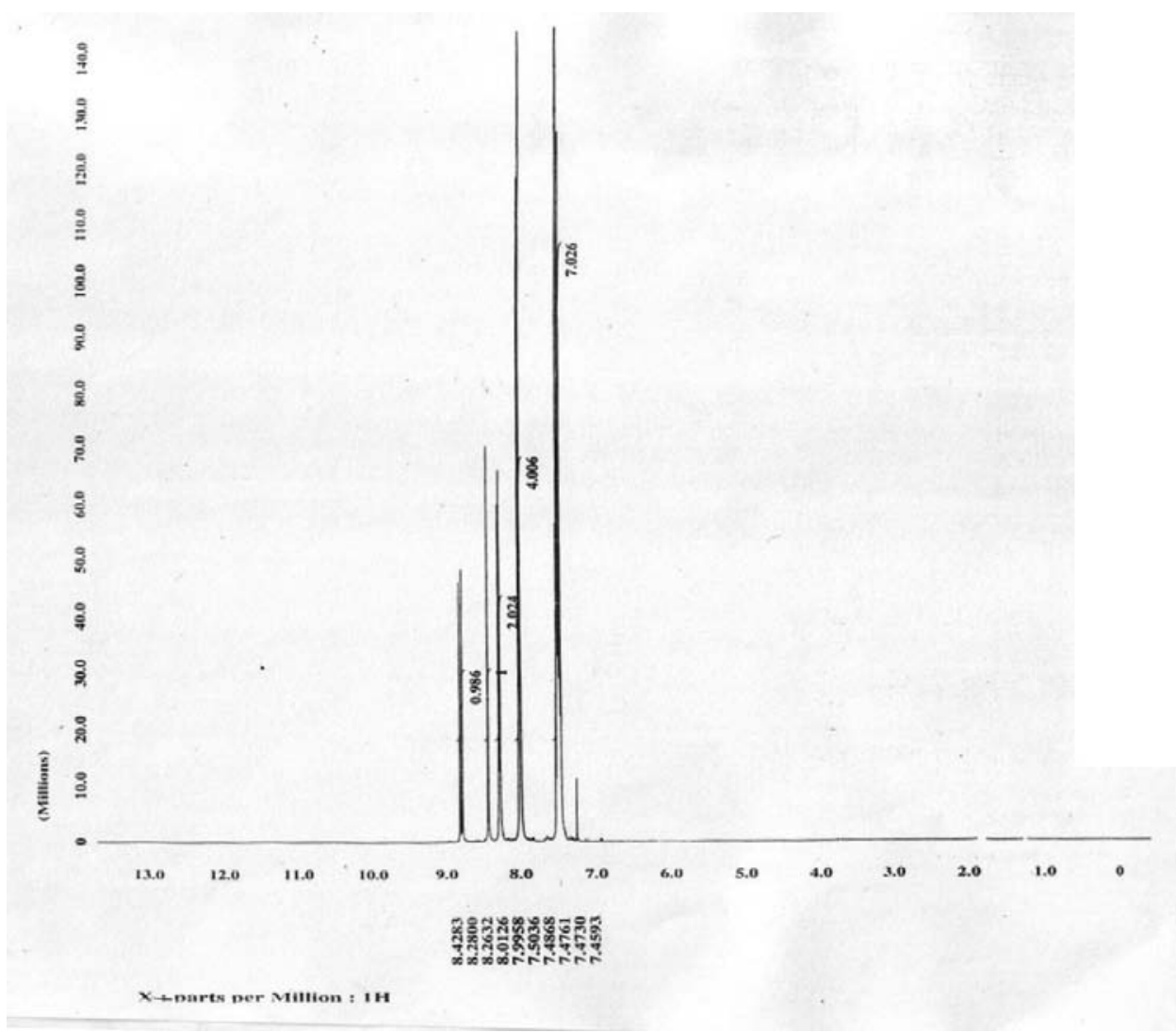

Figure S1. ${ }^{1} \mathrm{H}$ NMR spectrum of compound $\mathbf{1}$. 


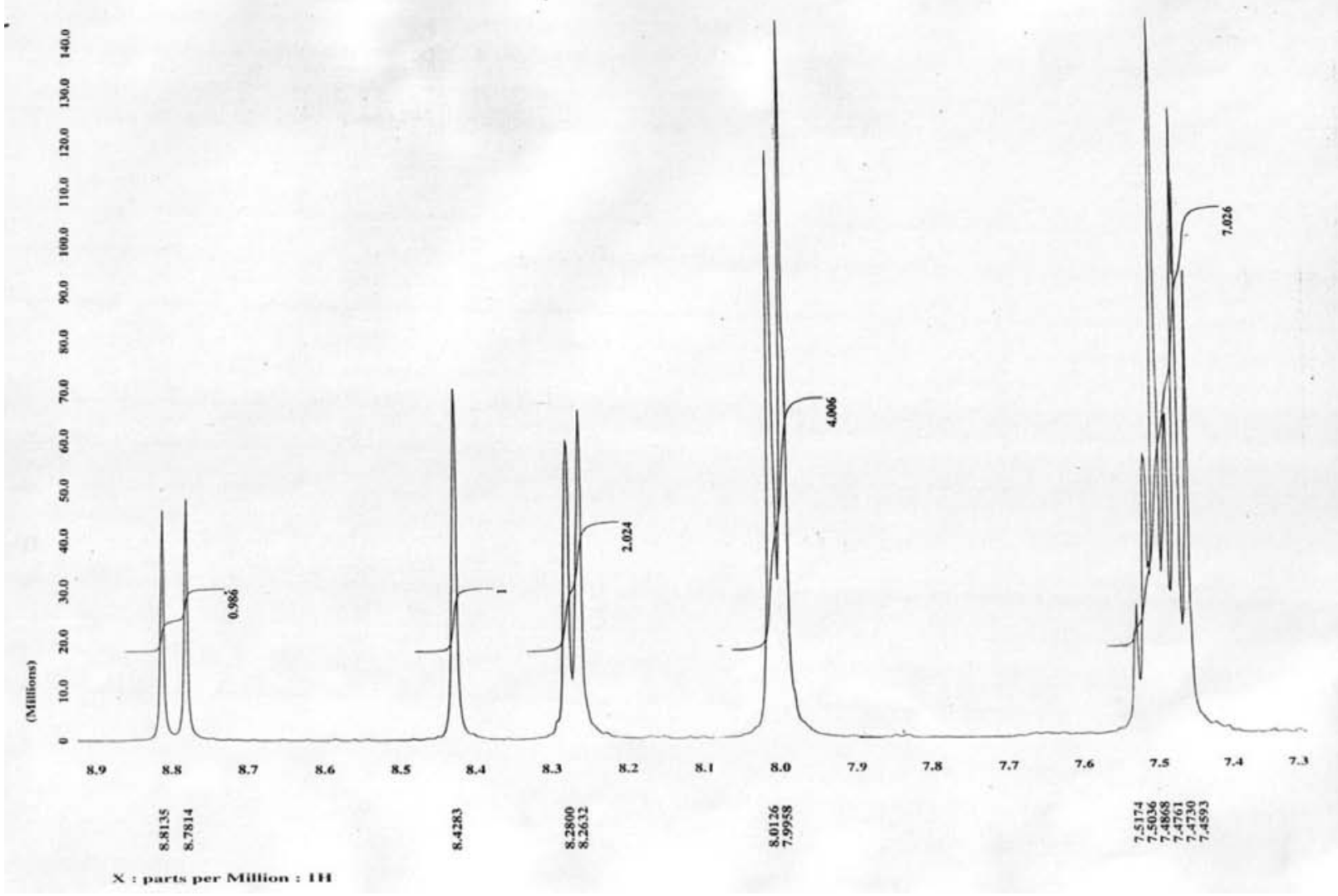

Figure S2. 'H NMR spectrum of compound 1.

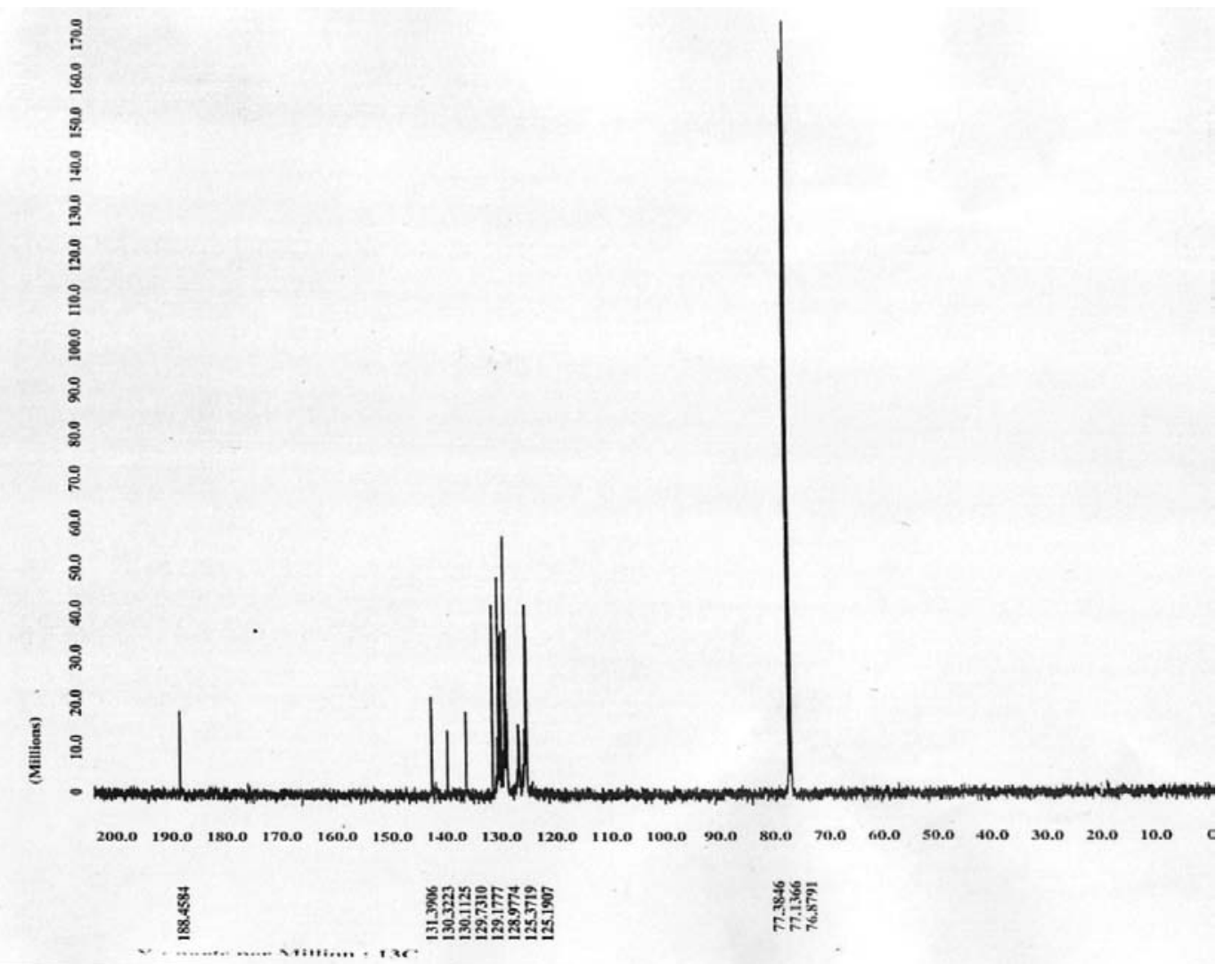

Figure S3. ${ }^{13} \mathrm{C}$ NMR spectrum of compound $\mathbf{1}$. 


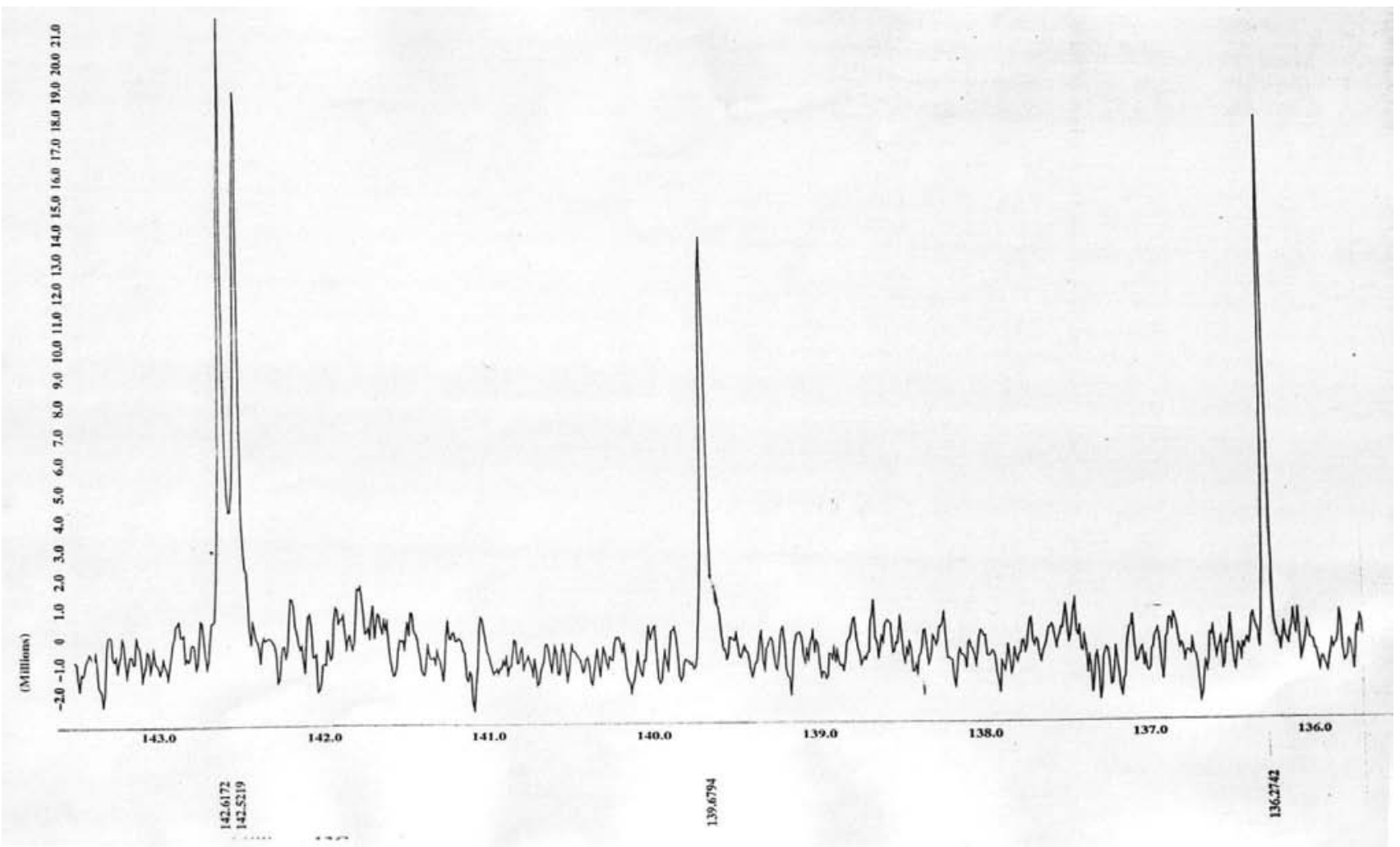

Figure S4. ${ }^{13} \mathrm{C}$ NMR spectrum of compound $\mathbf{1}$.

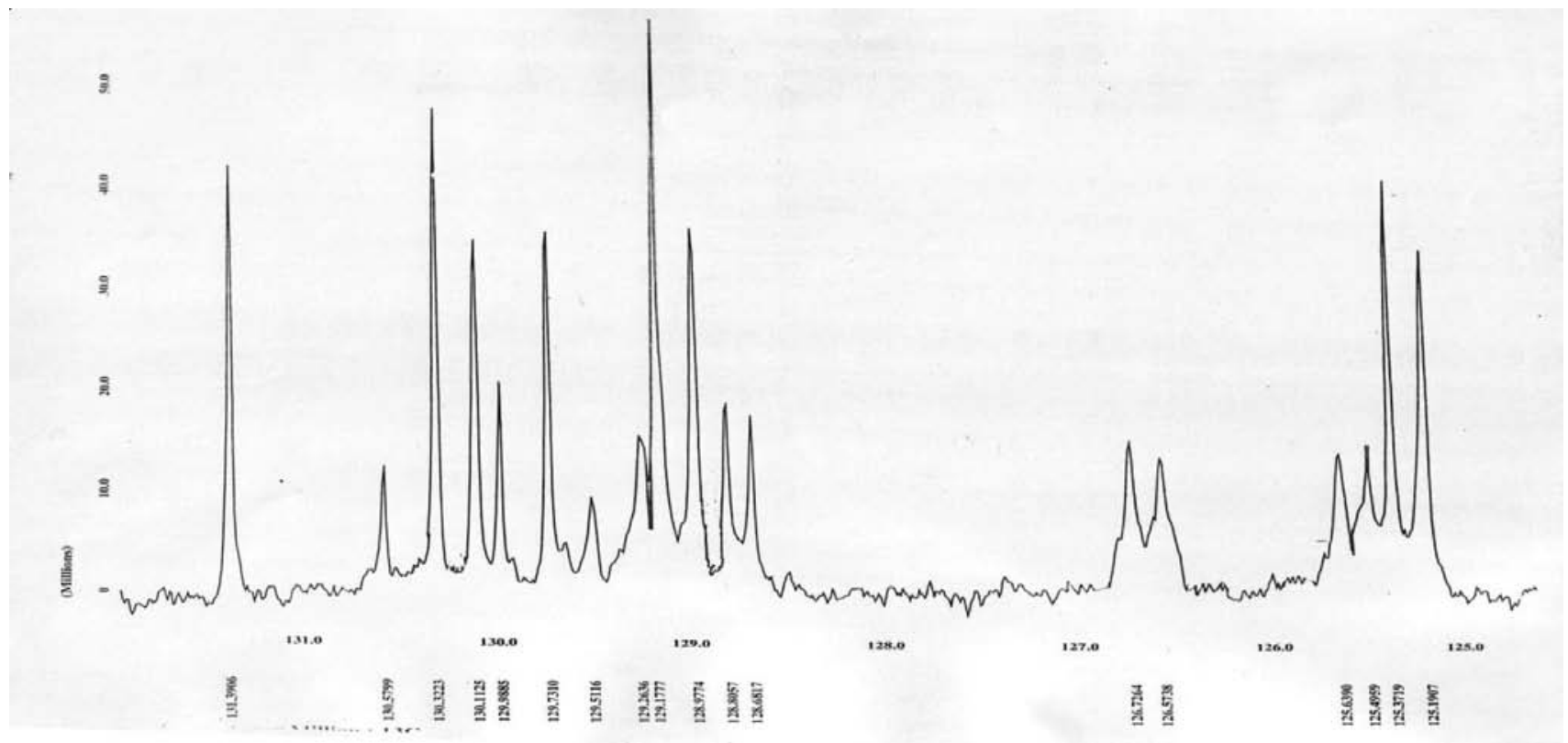

Figure S5. ${ }^{13} \mathrm{C}$ NMR spectrum of compound $\mathbf{1}$. 


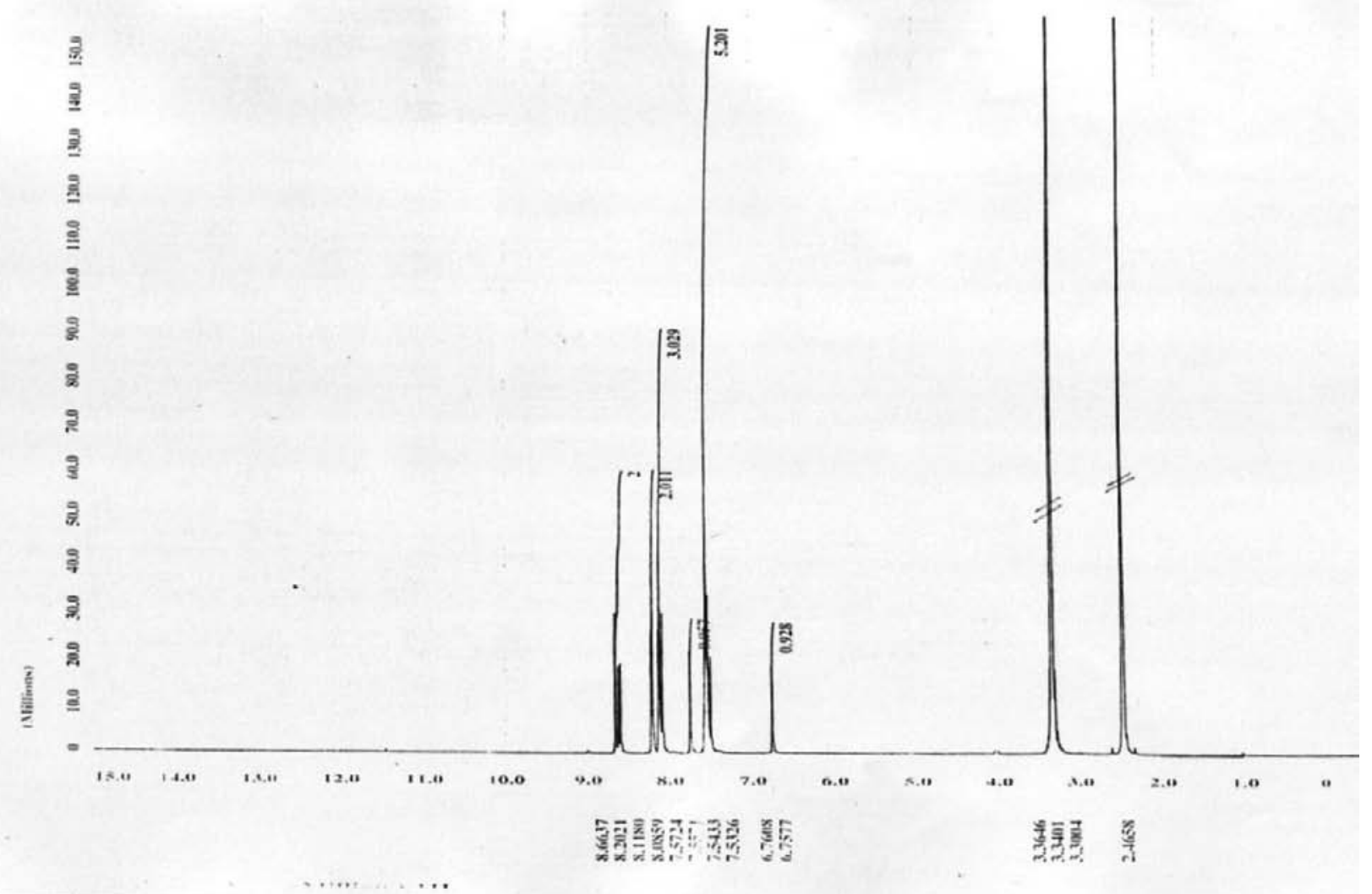

Figure S6. ${ }^{1} \mathrm{H}$ NMR spectrum of compound 3.

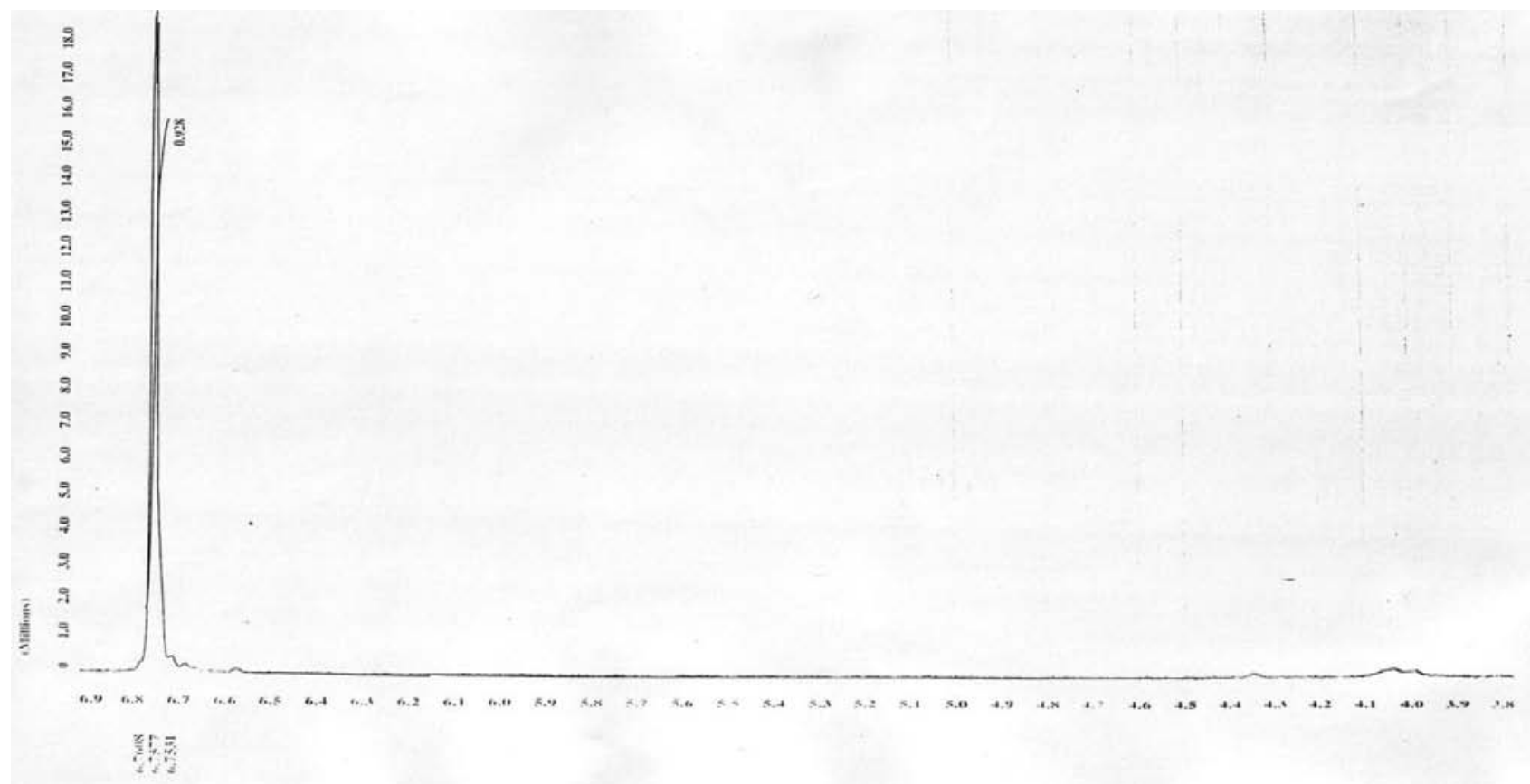

Figure S7. ${ }^{1} \mathrm{H}$ NMR spectrum of compound $\mathbf{3}$. 


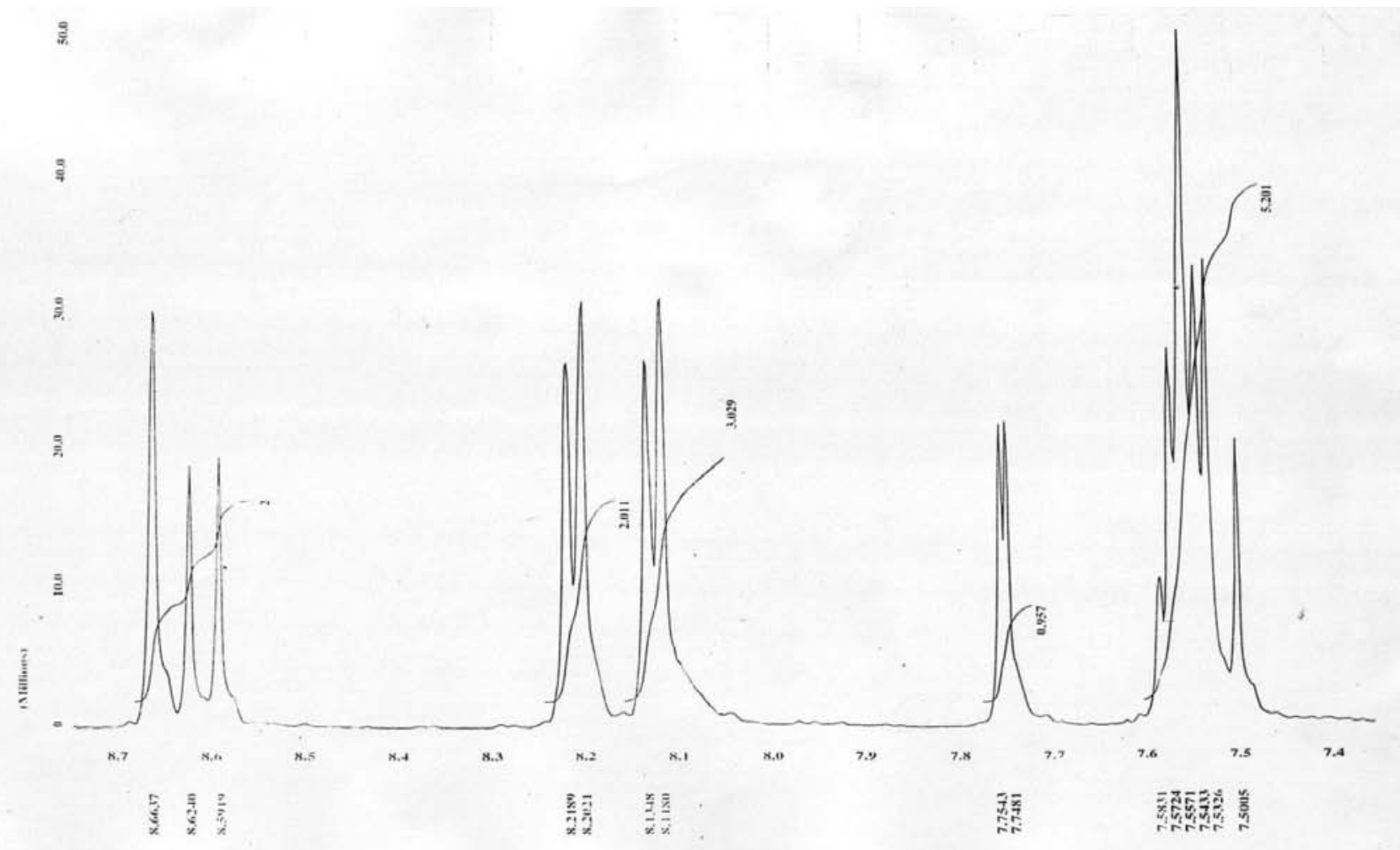

Figure S8. ${ }^{1} \mathrm{H}$ NMR spectrum of compound 3 .

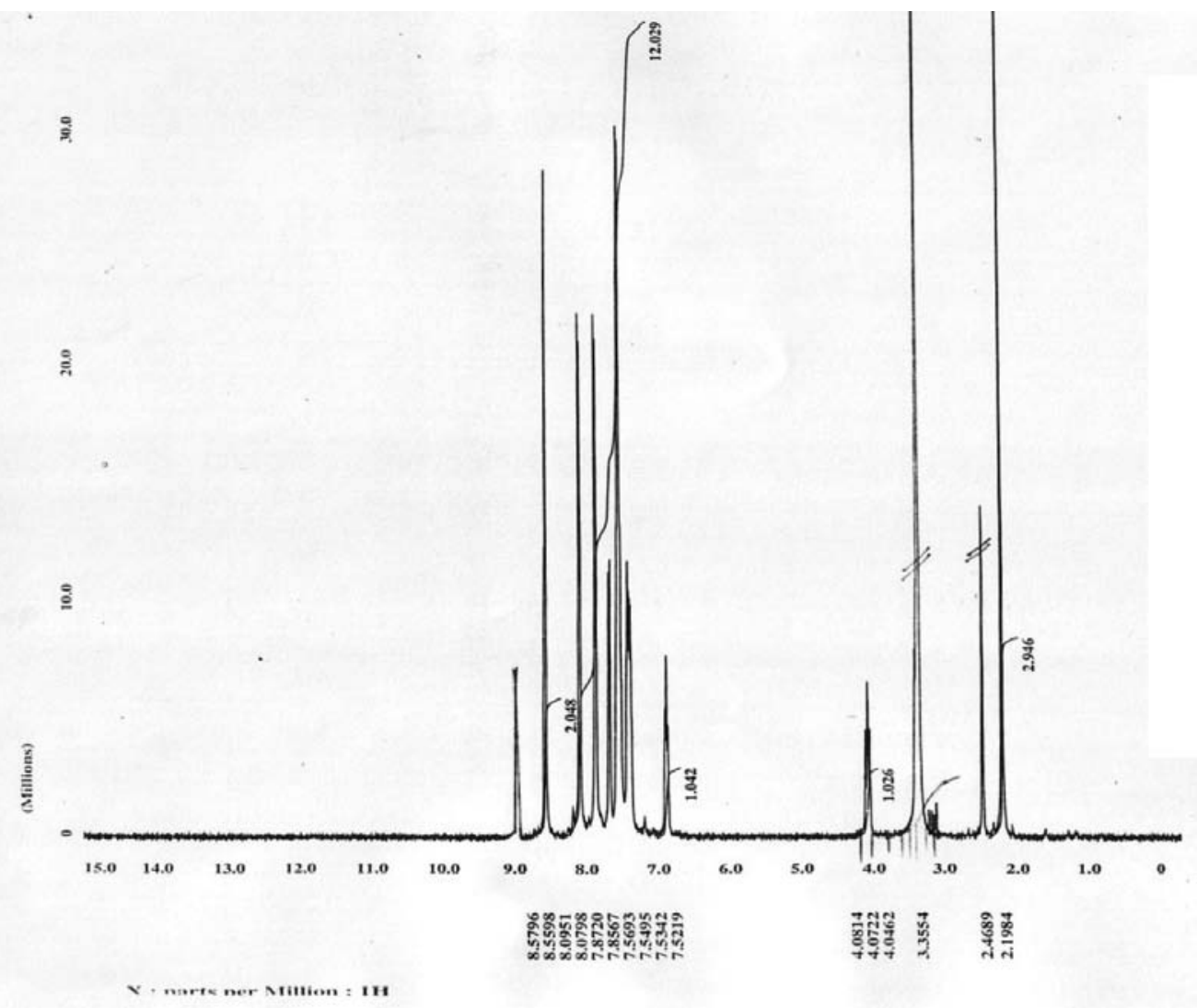

Figure S9. ${ }^{1} \mathrm{H}$ NMR spectrum of compound $\mathbf{5}$. 


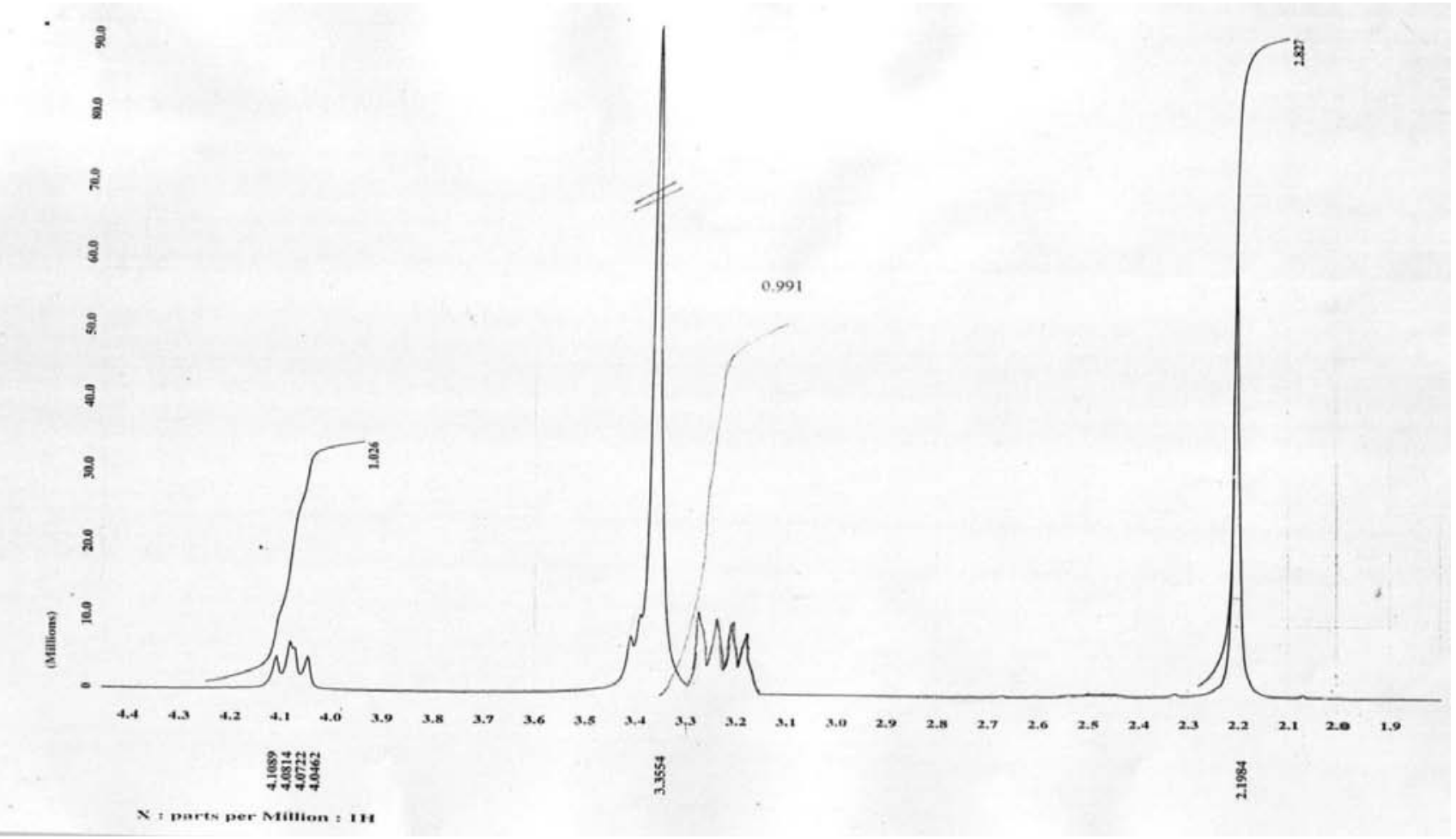

Figure S10. ${ }^{1} \mathrm{H}$ NMR spectrum of compound 5.

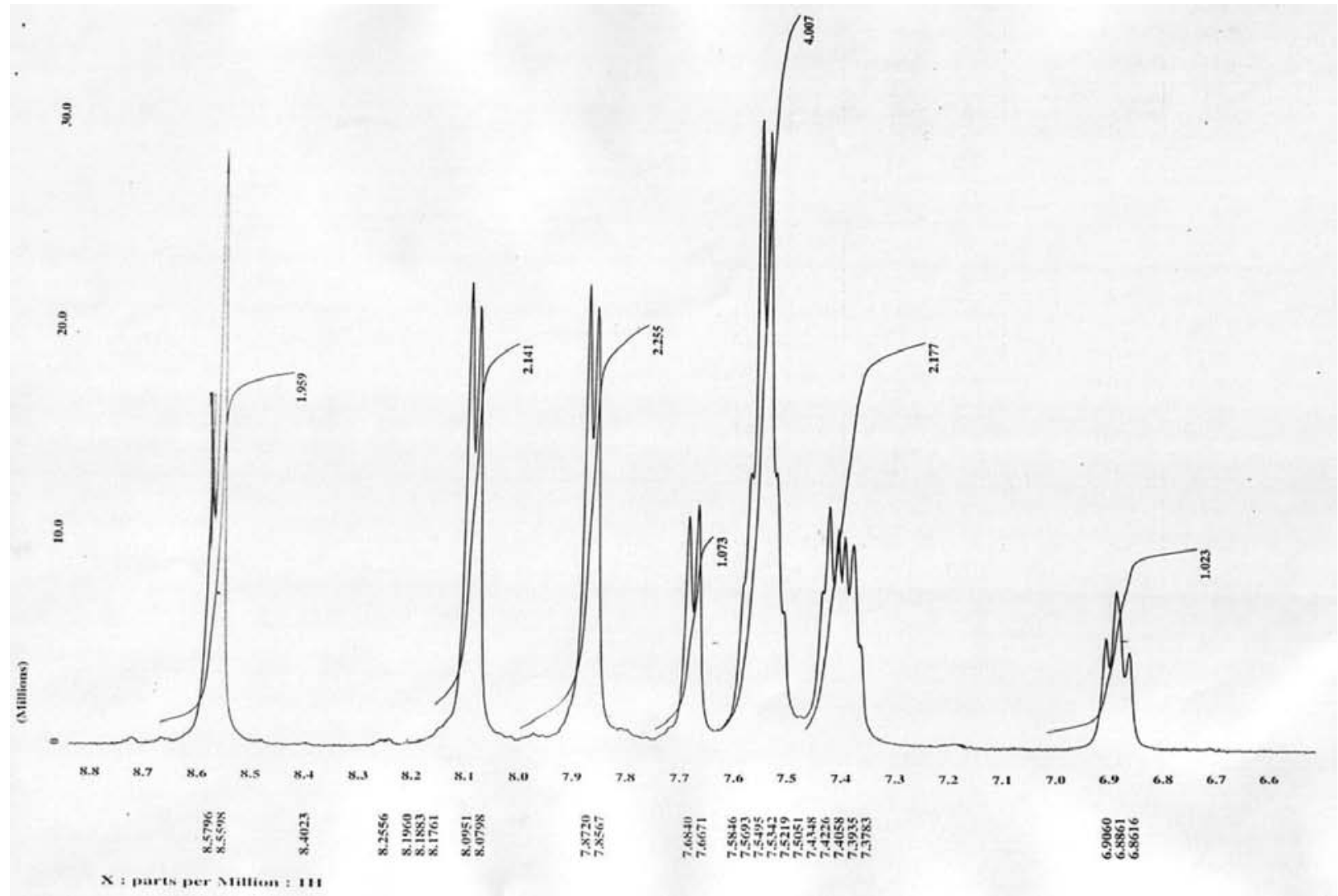

Figure S11. ${ }^{1} \mathrm{H}$ NMR spectrum of compound 5. 


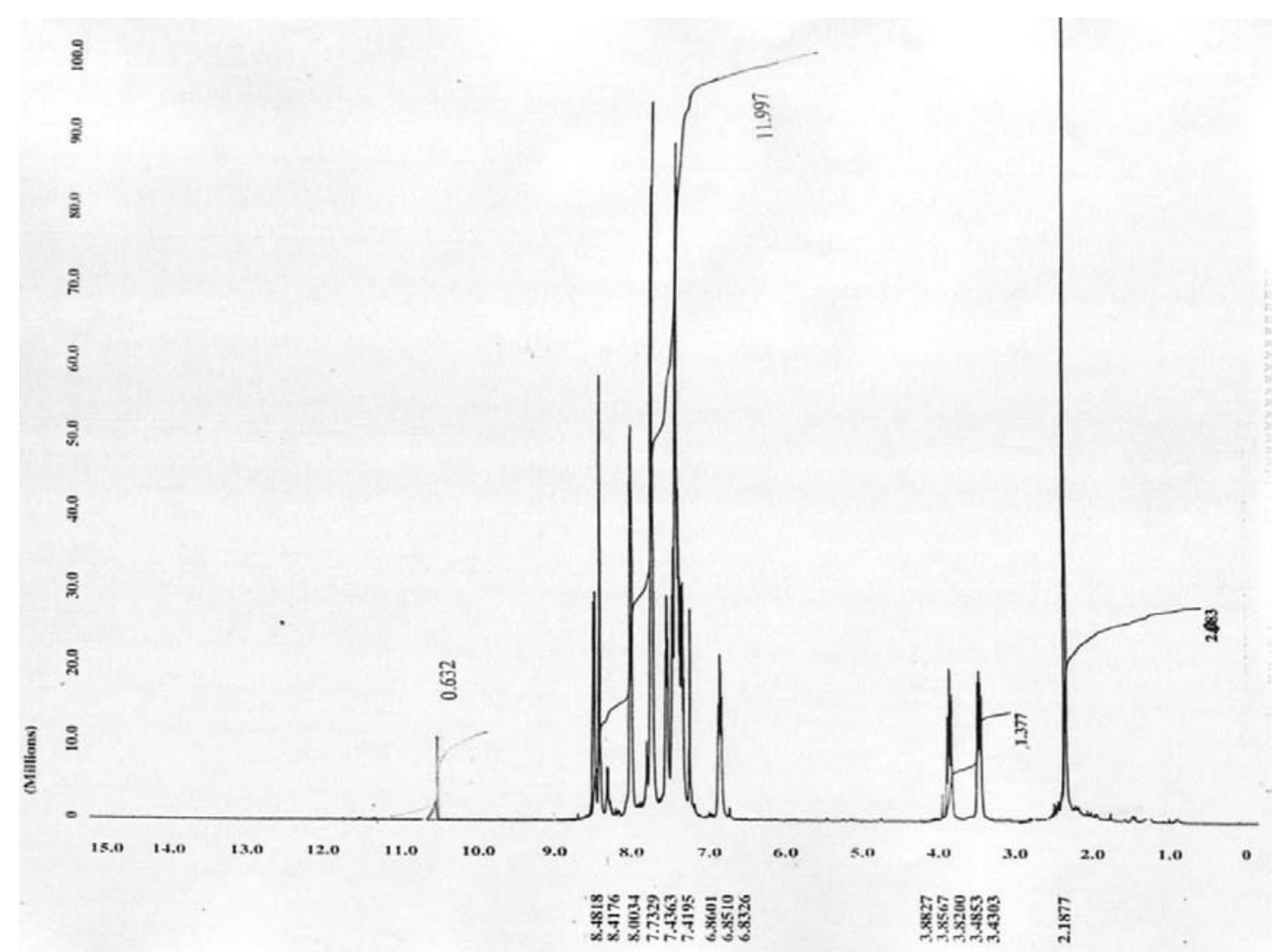

Figure S12. ${ }^{1} \mathrm{H}$ NMR spectrum of compound $\mathbf{8}$.

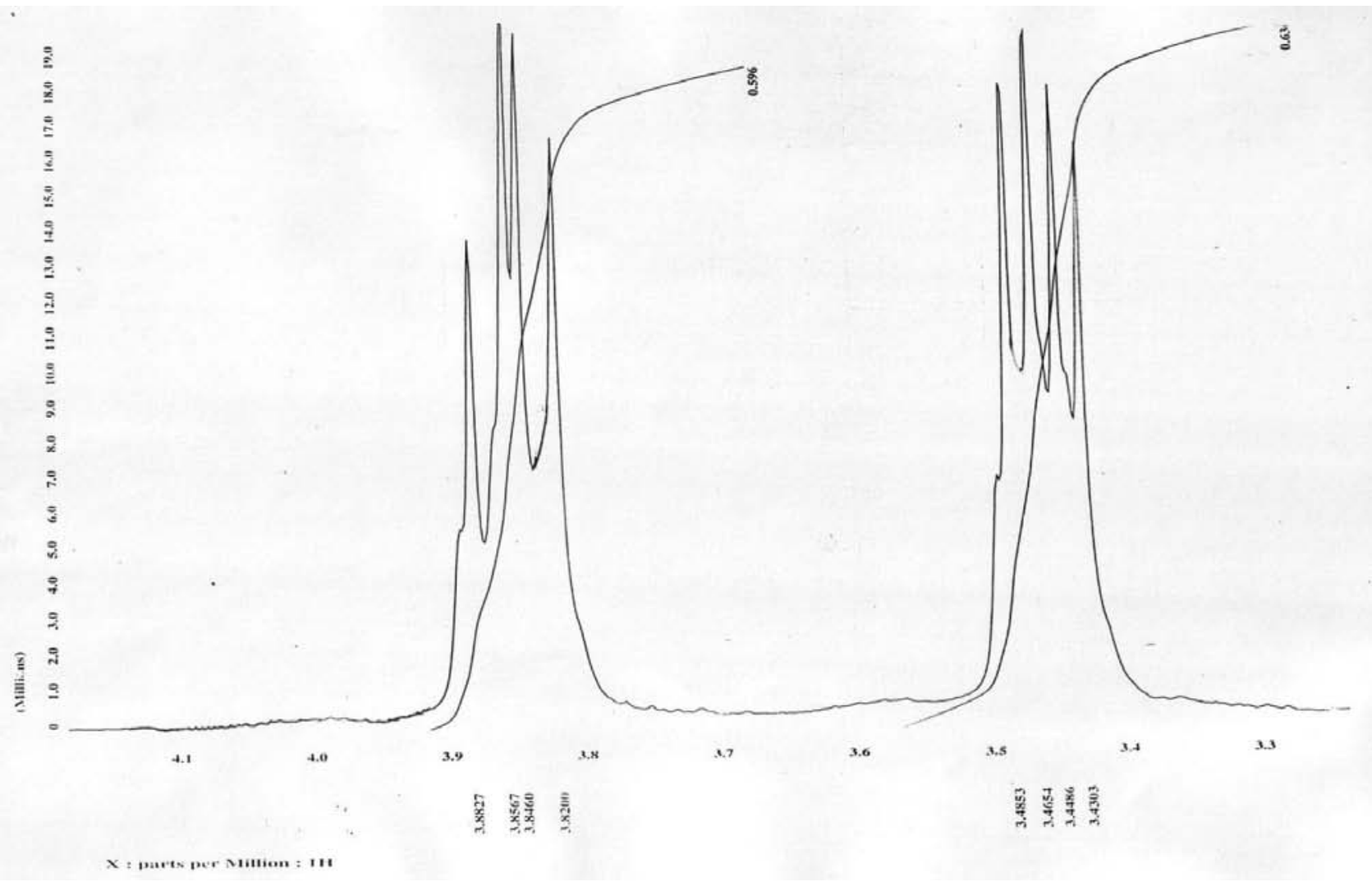

Figure S13. ${ }^{1} \mathrm{H}$ NMR spectrum of compound 8. 


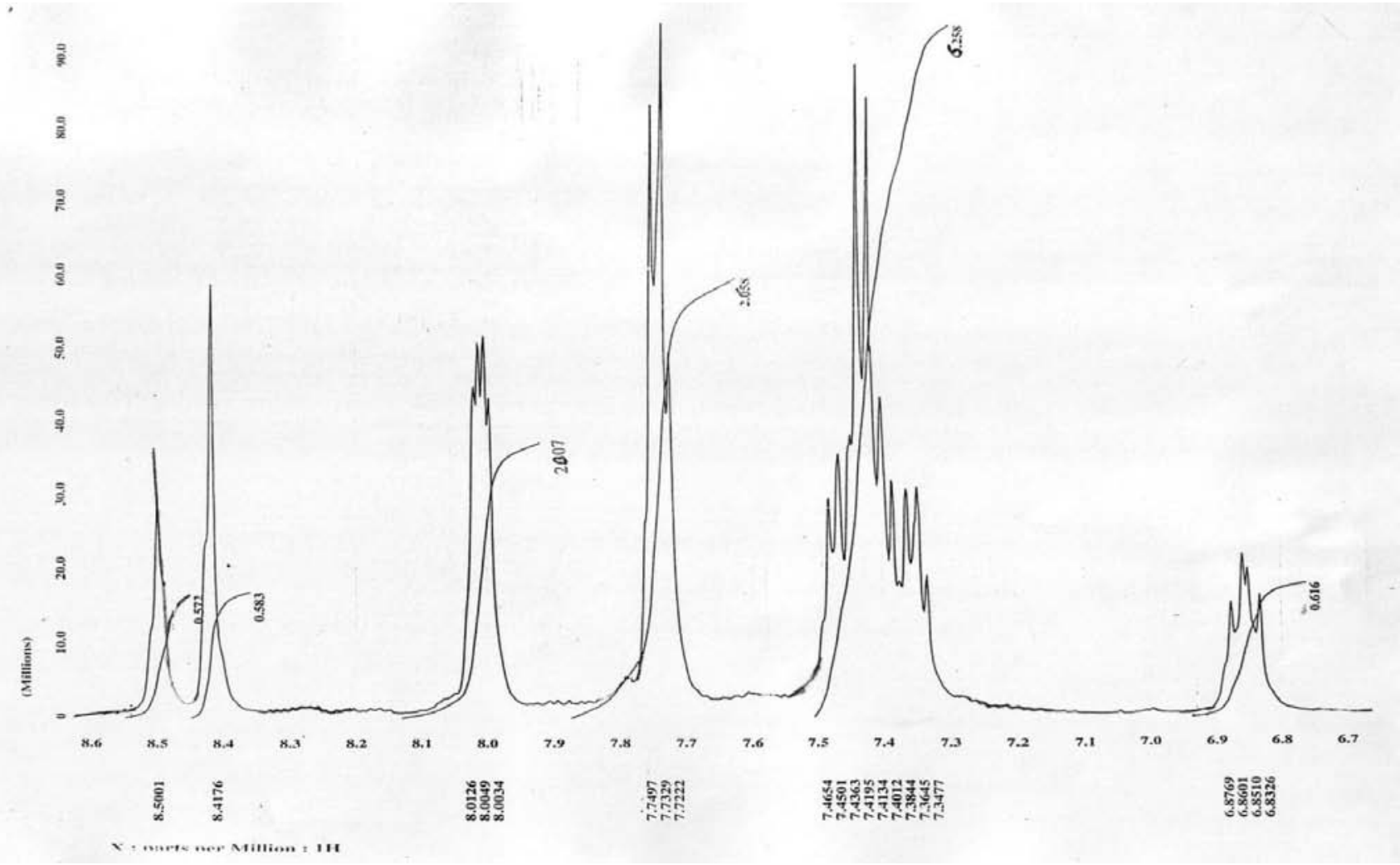

Figure S14. ${ }^{1} \mathrm{H}$ NMR spectrum of compound 8 .

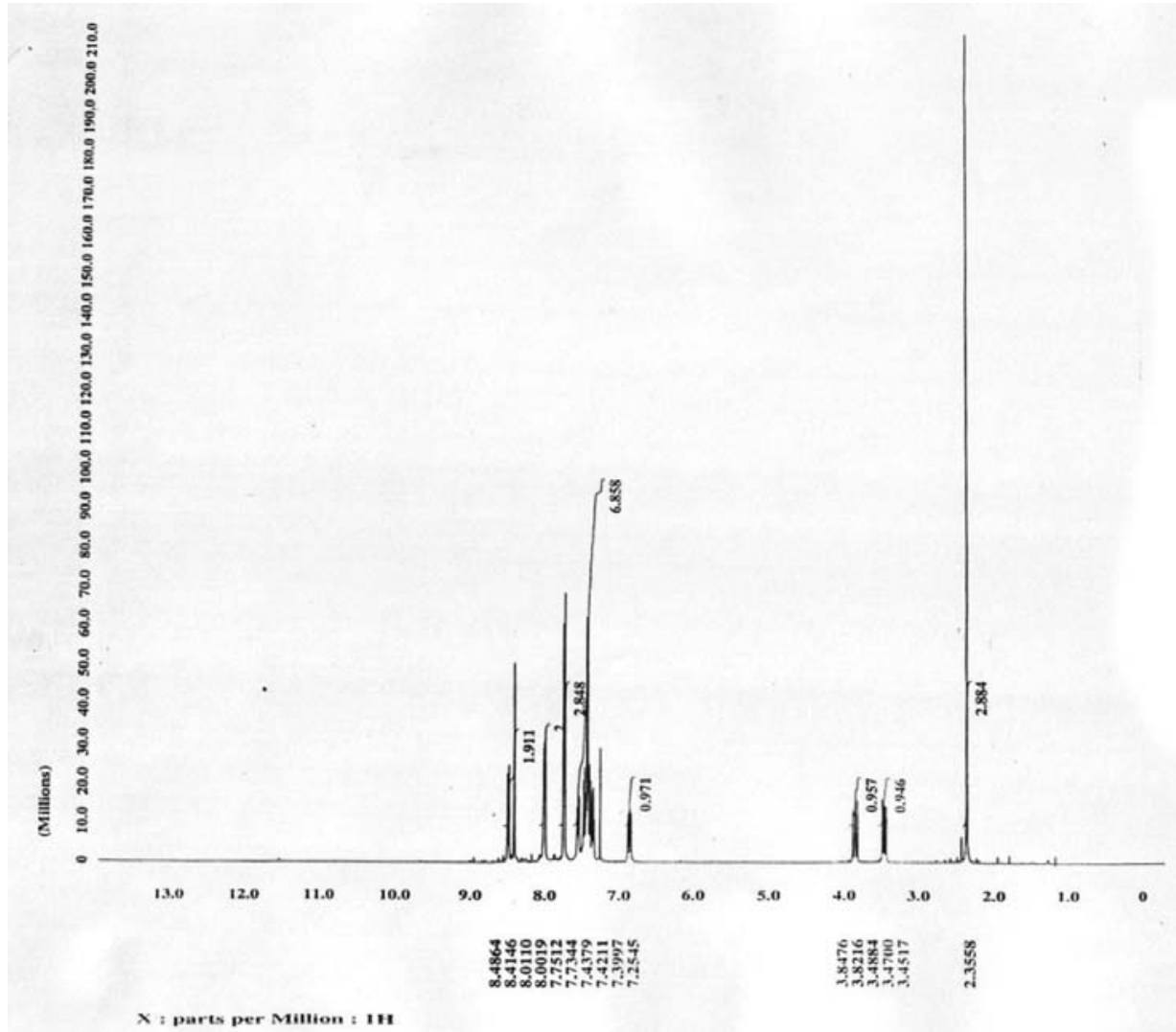

Figure S15. ${ }^{1} \mathrm{H}$ NMR spectrum of compound 9. 


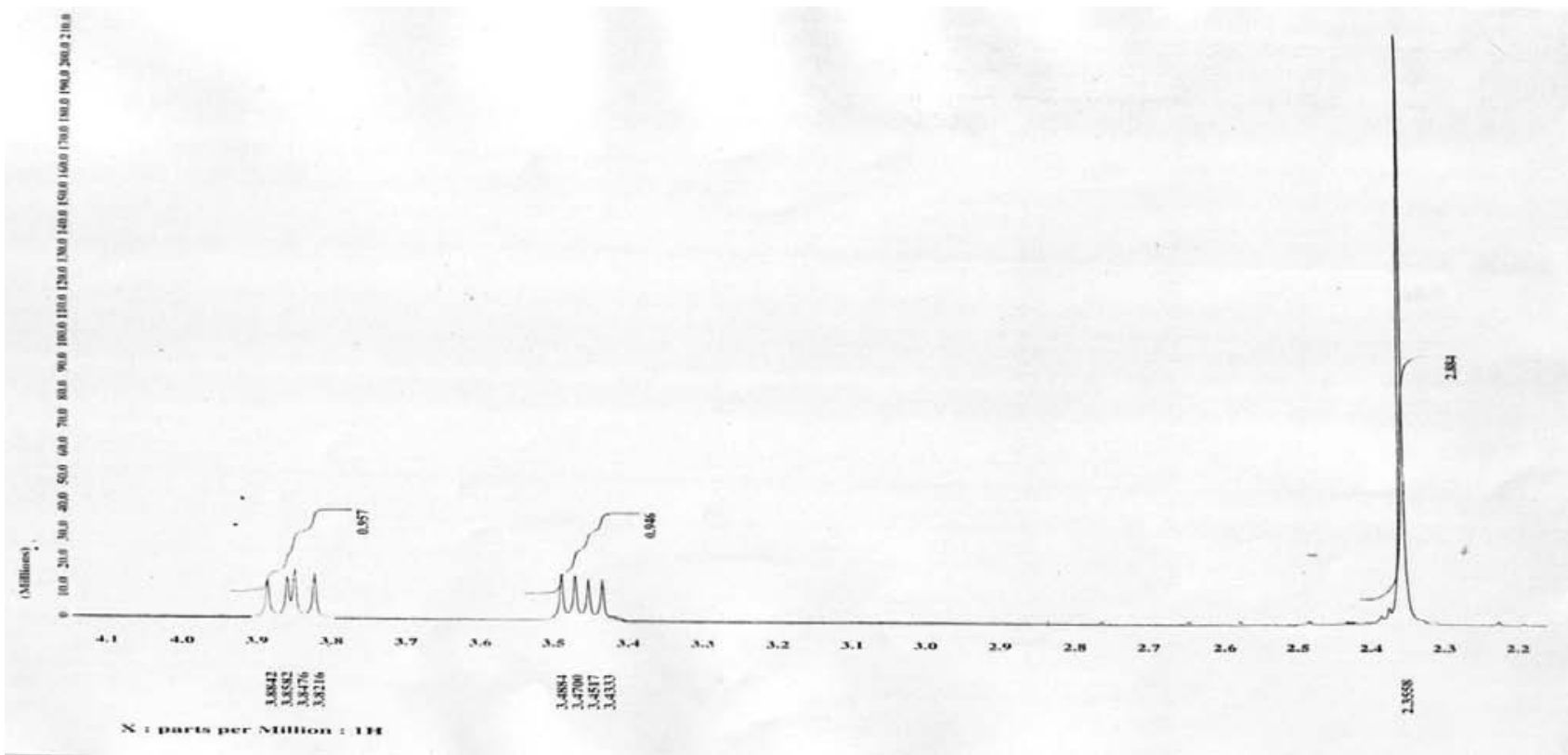

Figure S16. ${ }^{1} \mathrm{H}$ NMR spectrum of compound 9.

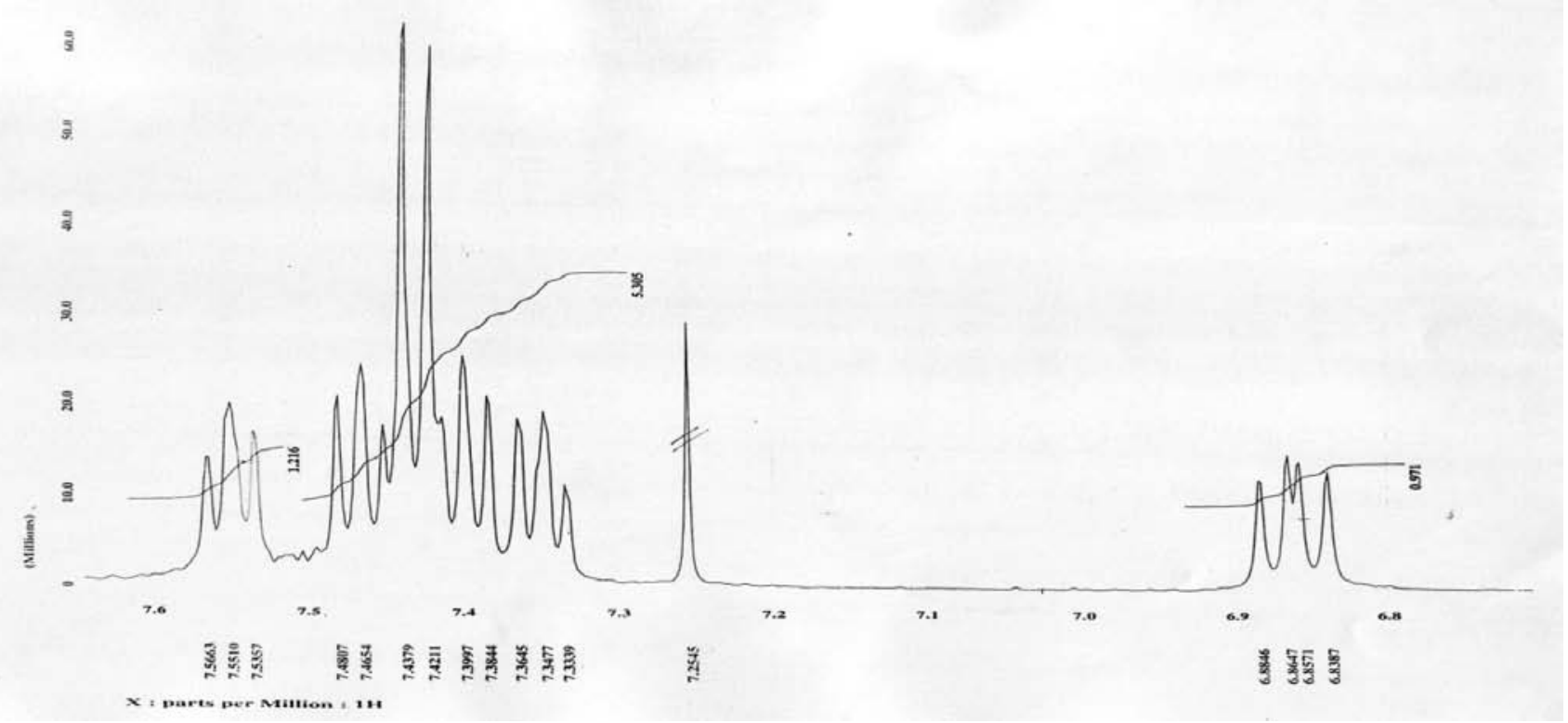

Figure S17. ${ }^{1} \mathrm{H}$ NMR spectrum of compound 9. 


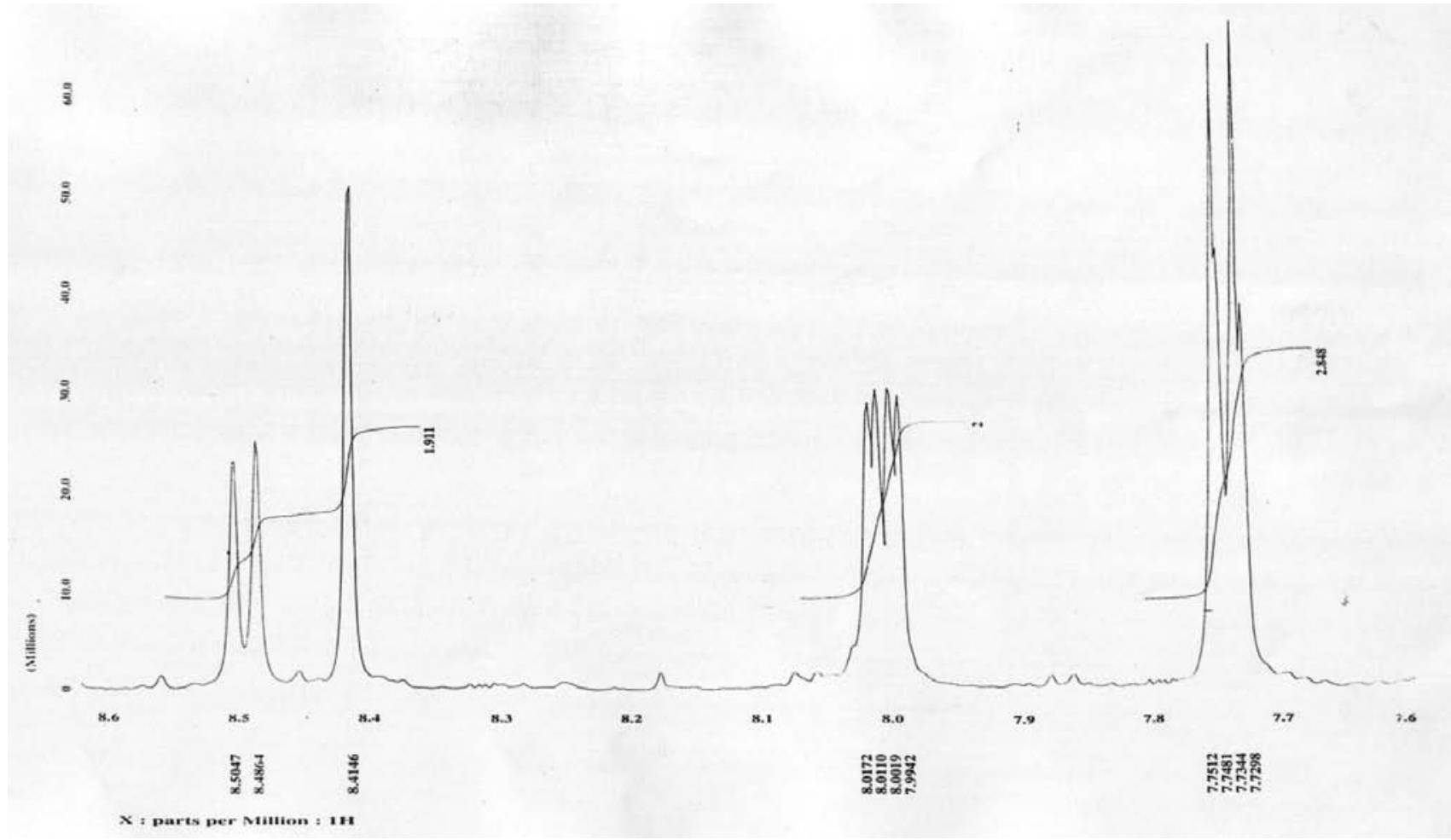

Figure S18. ${ }^{1} \mathrm{H}$ NMR spectrum of compound 9.

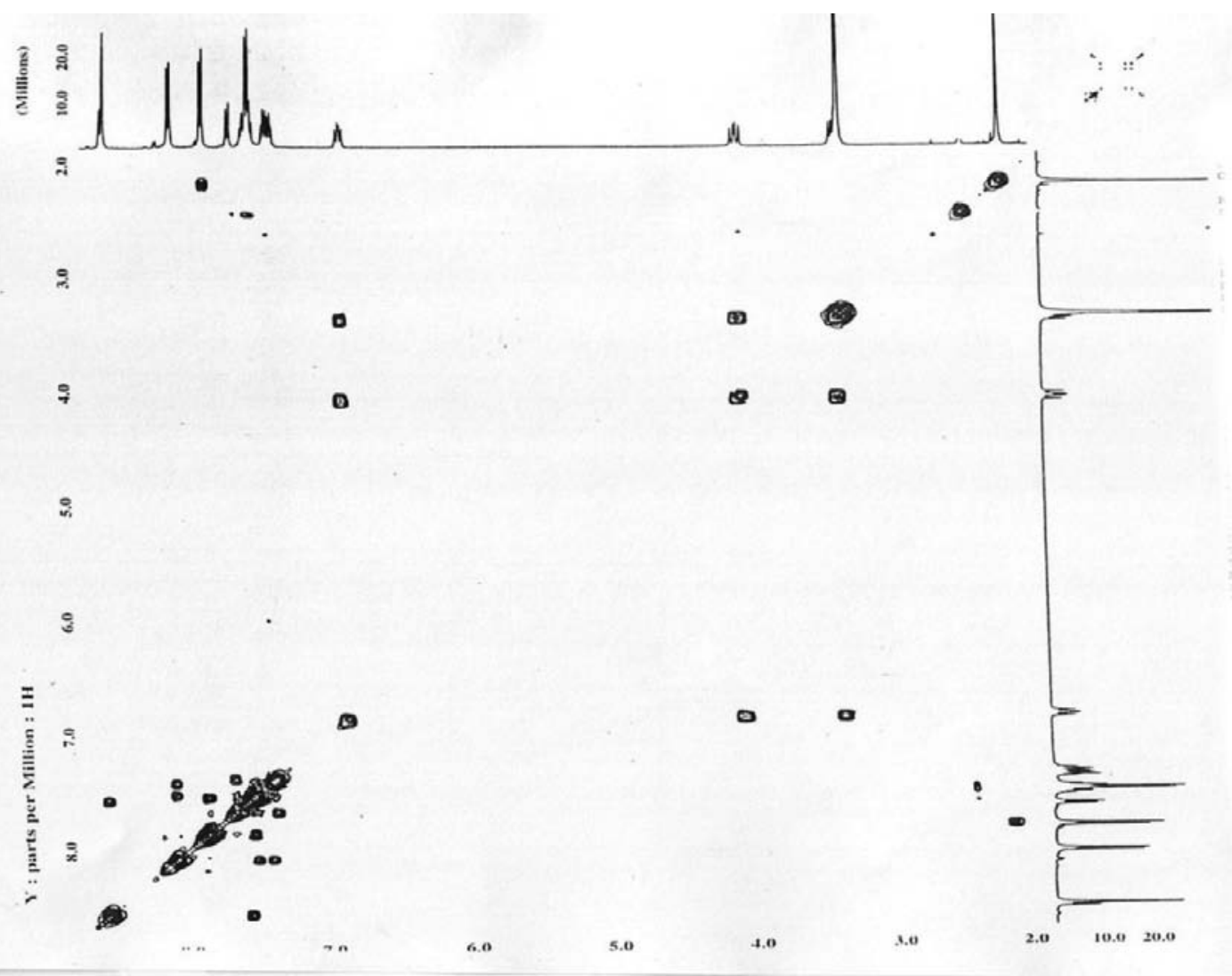

Figure S19. 2D NMR spectrum of compound 9 


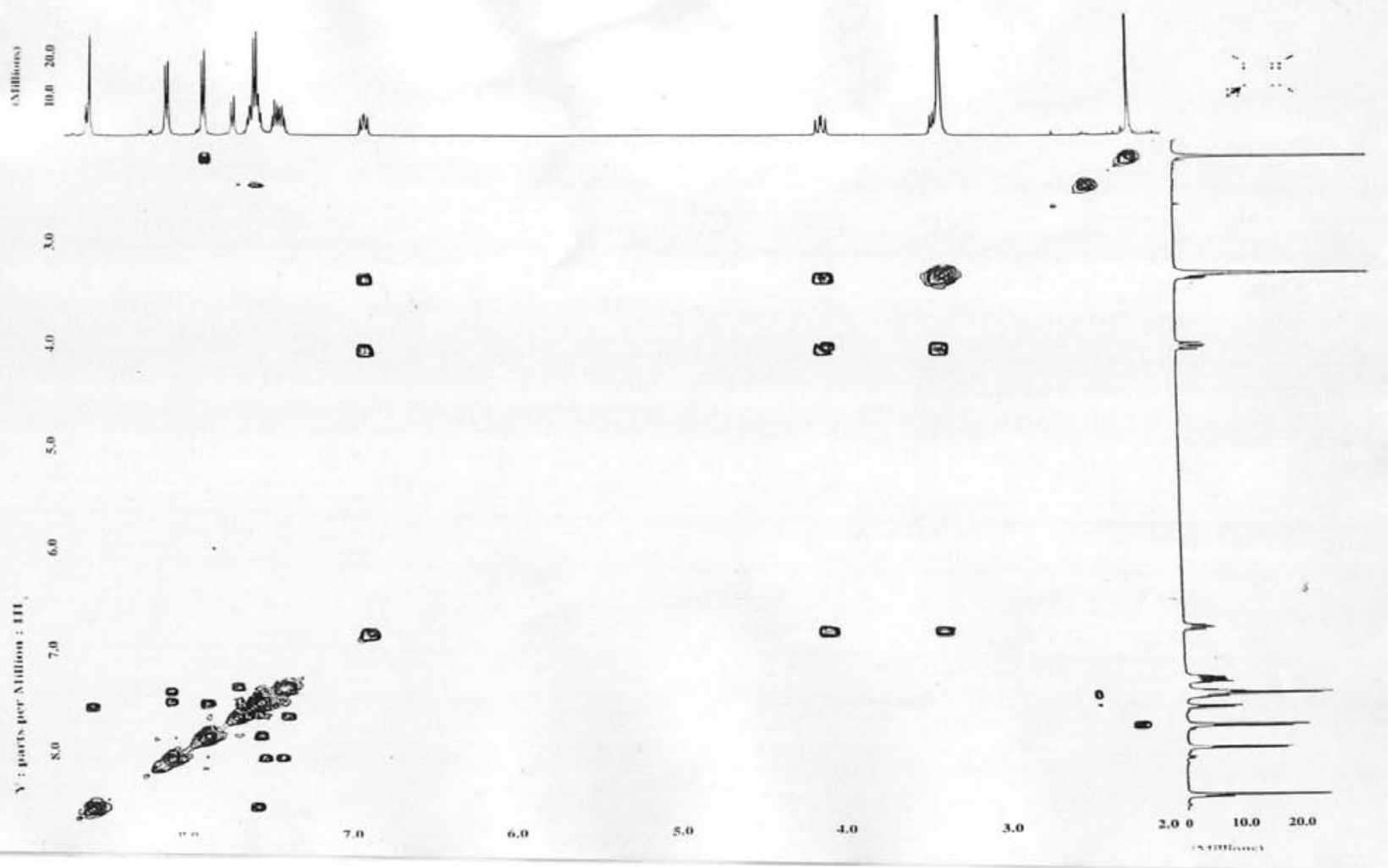

Figure S20. 2D NMR spectrum of compound 9.

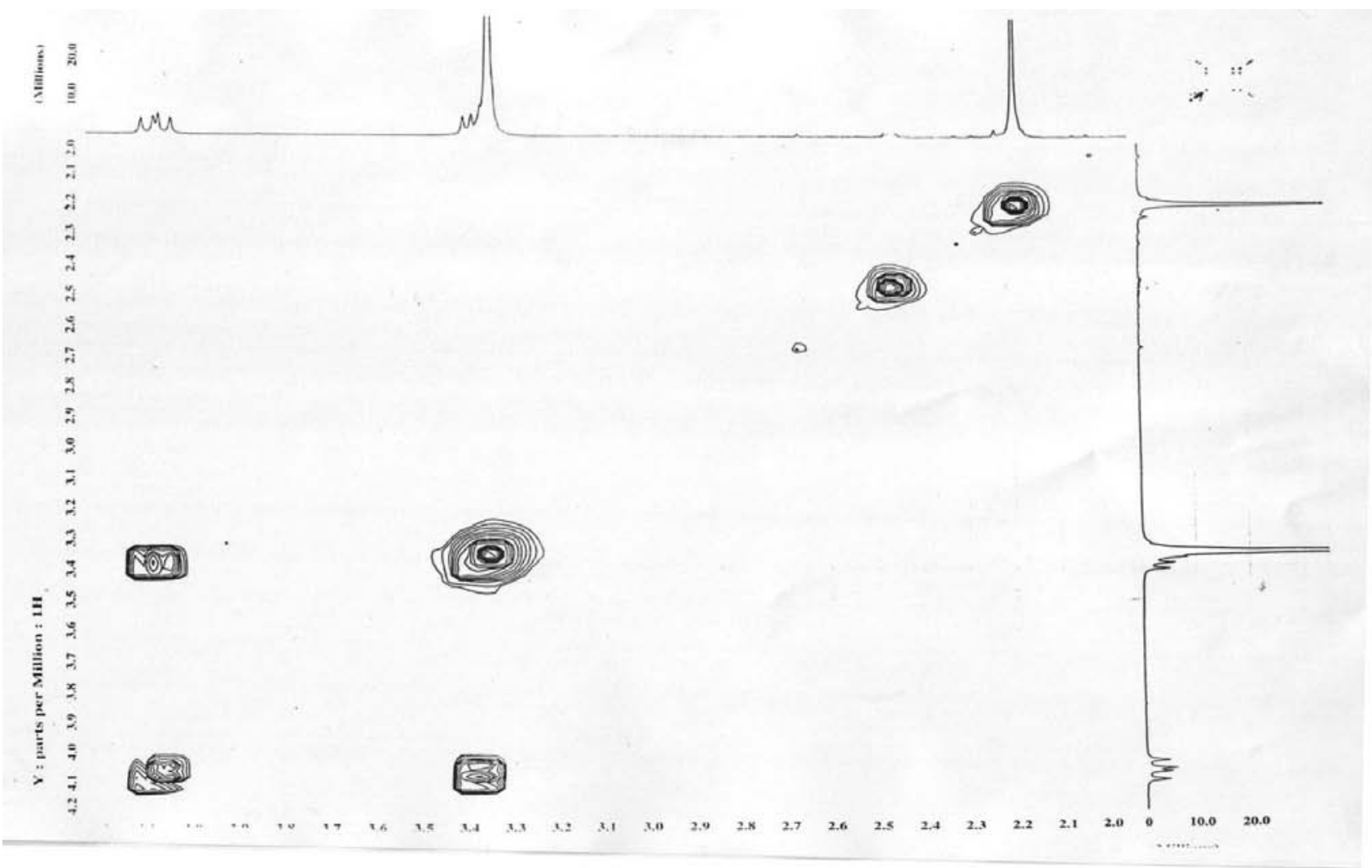

Figure S21. 2D NMR spectrum of compound 9. 


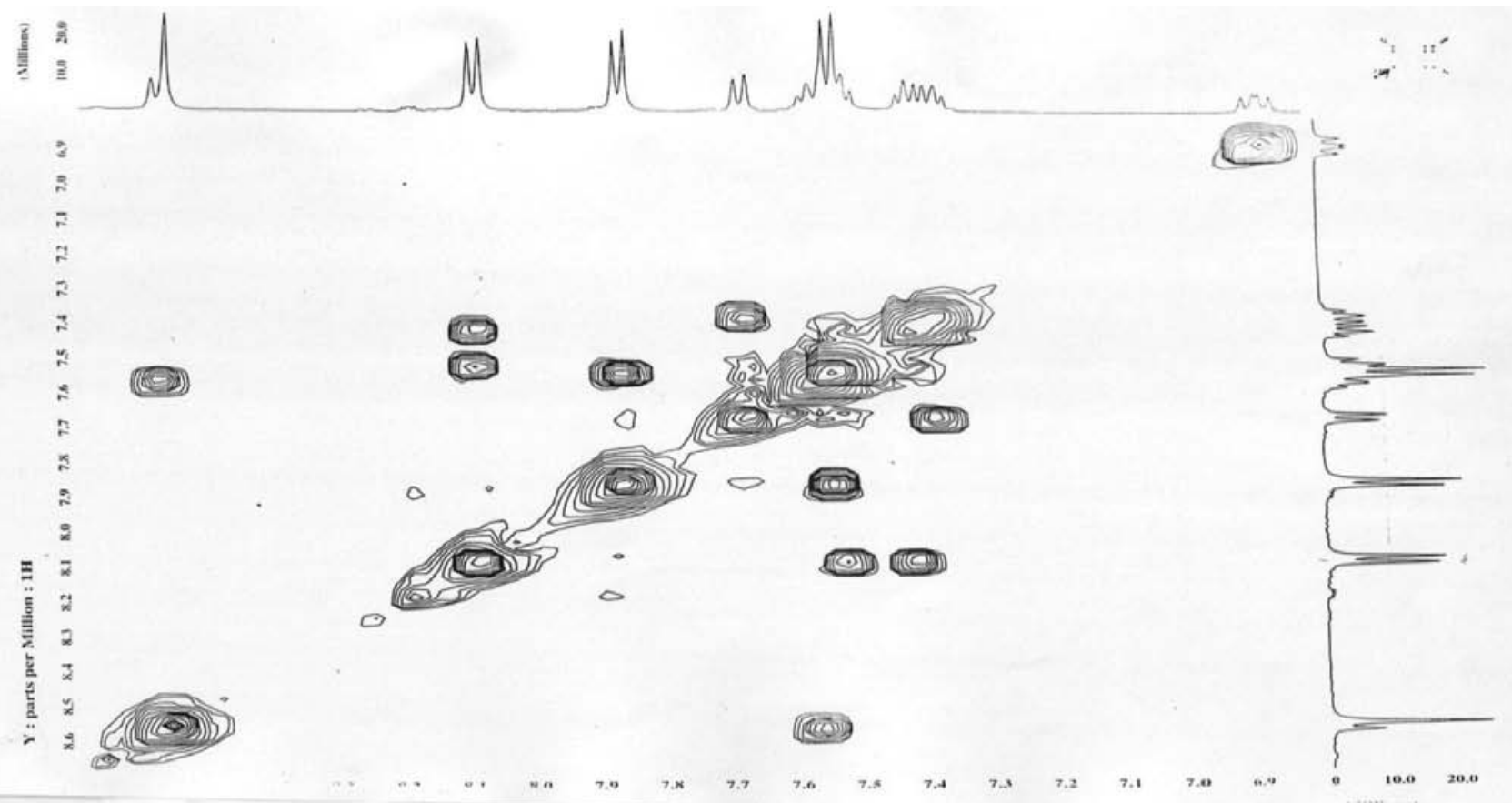

Figure S22. 2D NMR spectrum of compound 9.

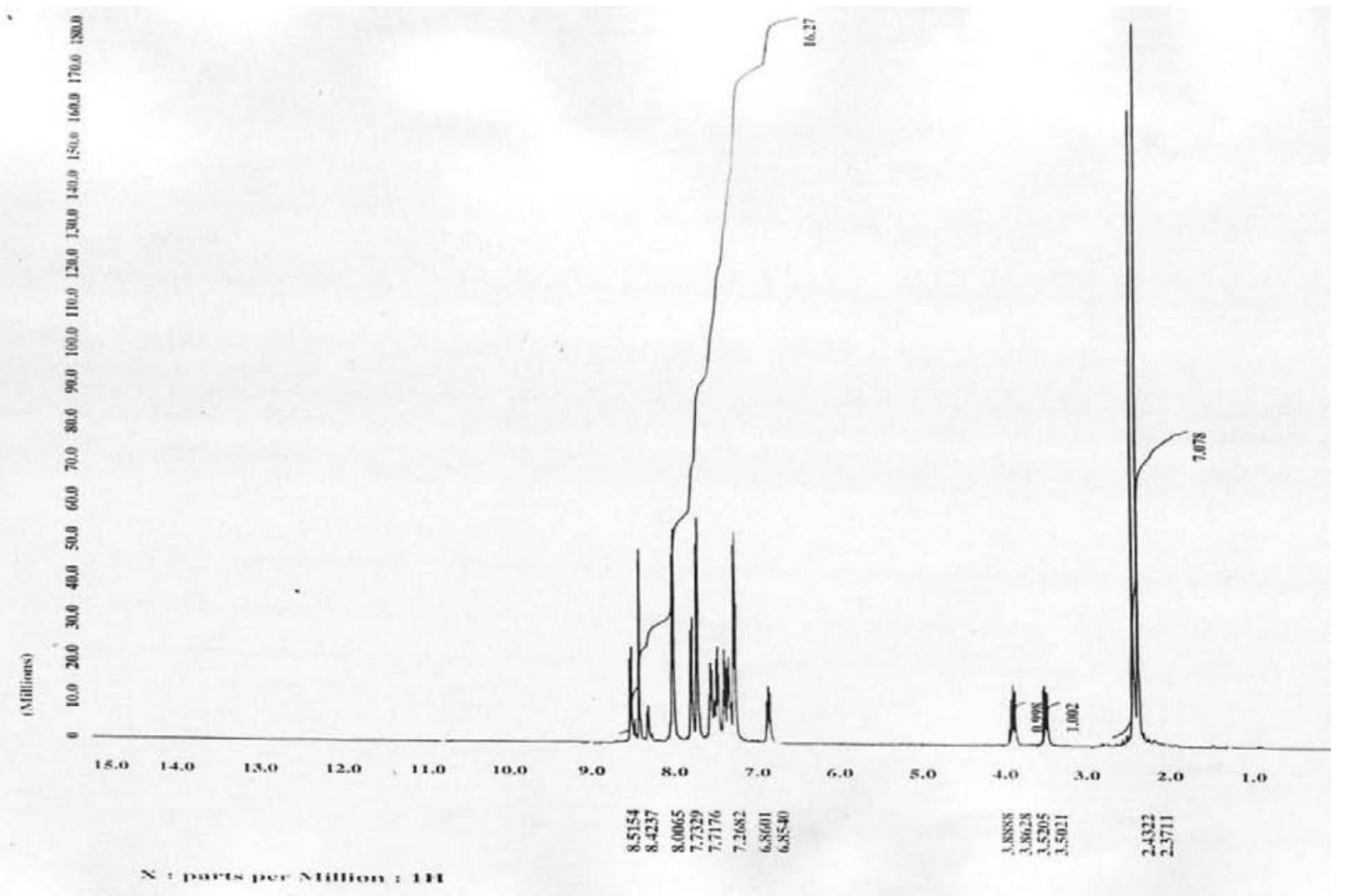

Figure S23. ${ }^{1} \mathrm{H}$ NMR spectrum of compound $\mathbf{1 0}$. 


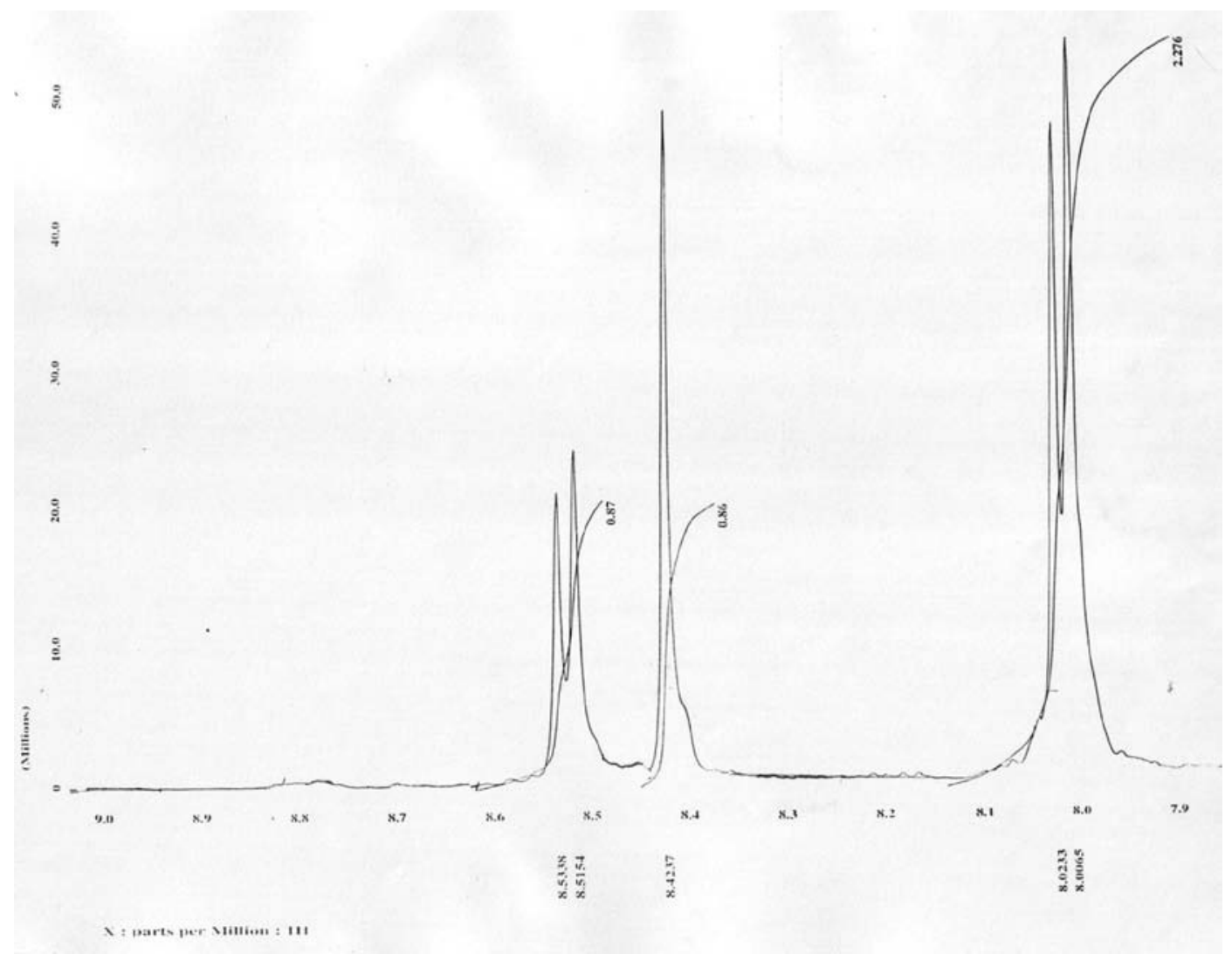

Figure S24. ${ }^{1} \mathrm{H}$ NMR spectrum of compound $\mathbf{1 0}$.

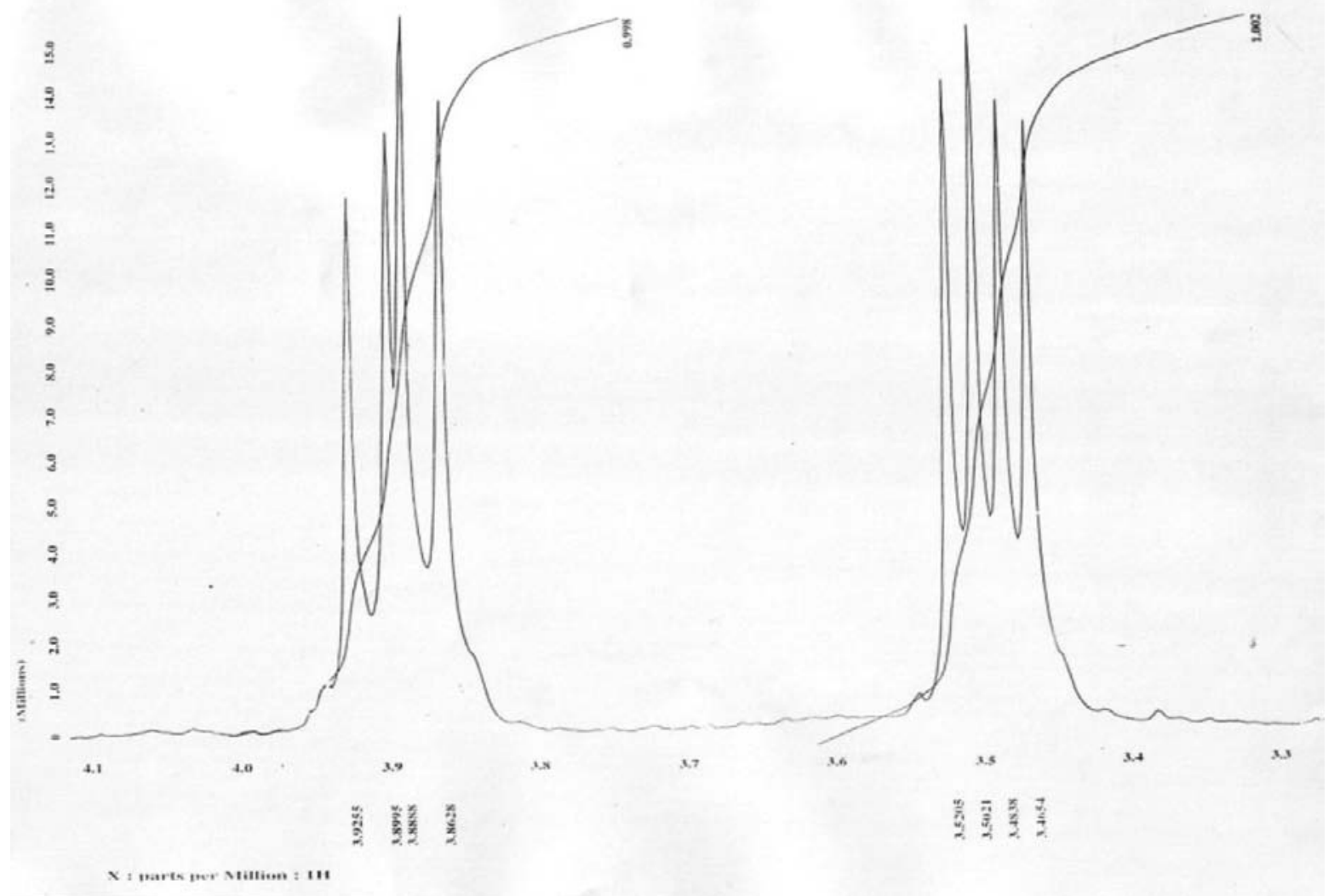

Figure S25. ${ }^{1} \mathrm{H}$ NMR spectrum of compound $\mathbf{1 0}$. 


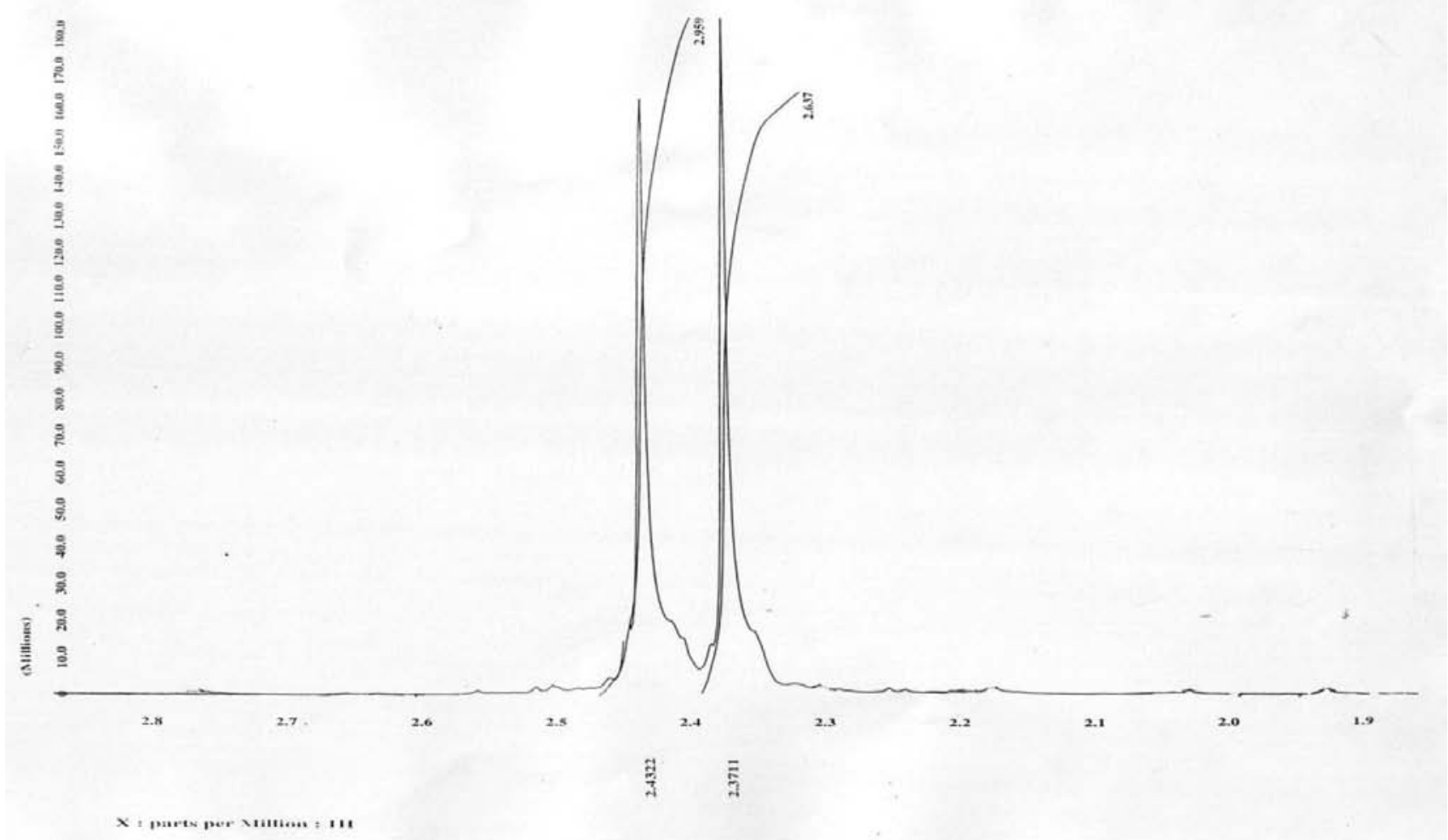

Figure S26. ${ }^{1} \mathrm{H}$ NMR spectrum of compound $\mathbf{1 0}$.

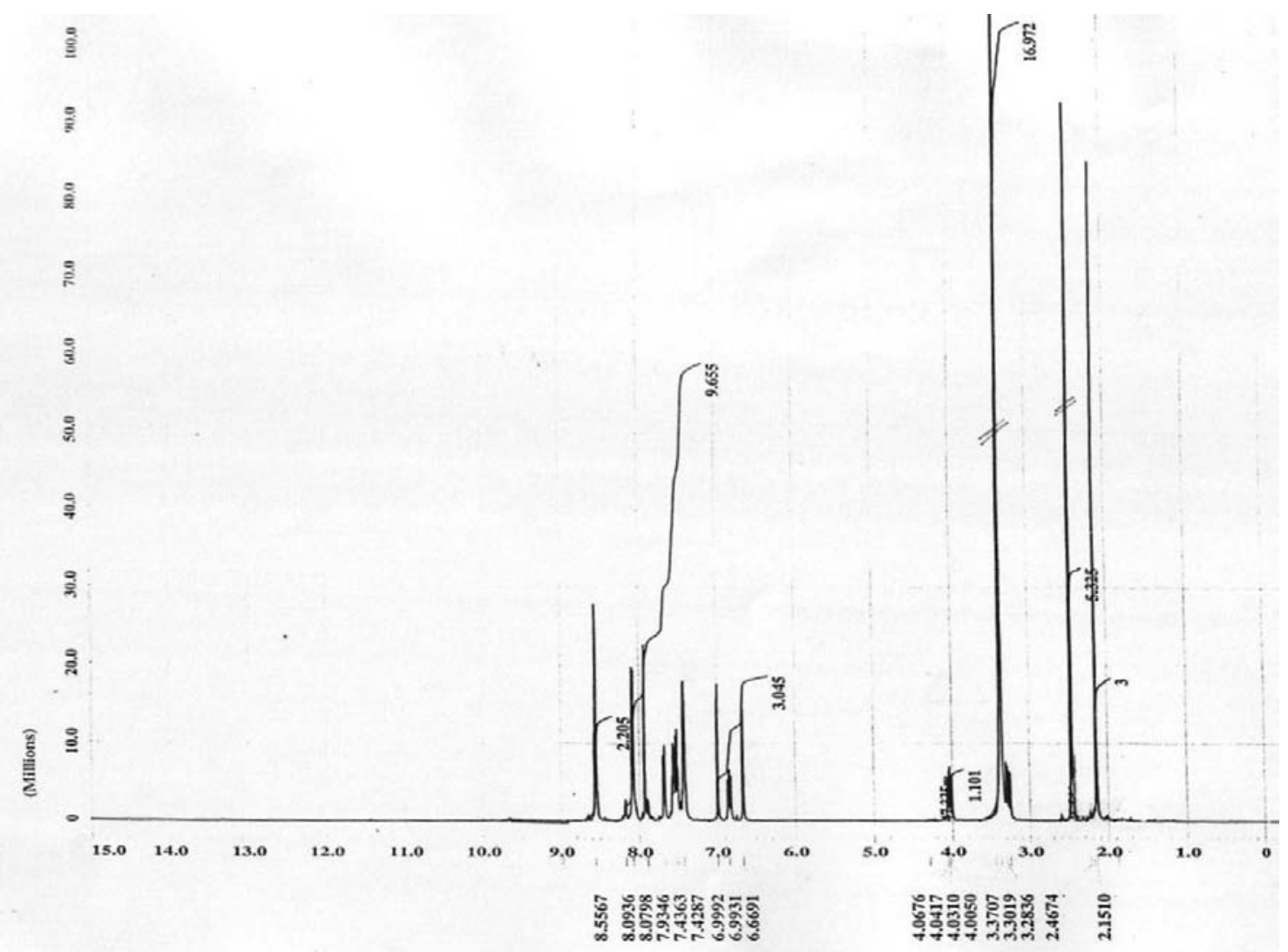

Figure S27. ${ }^{1} \mathrm{H}$ NMR spectrum of compound $\mathbf{1 1}$. 


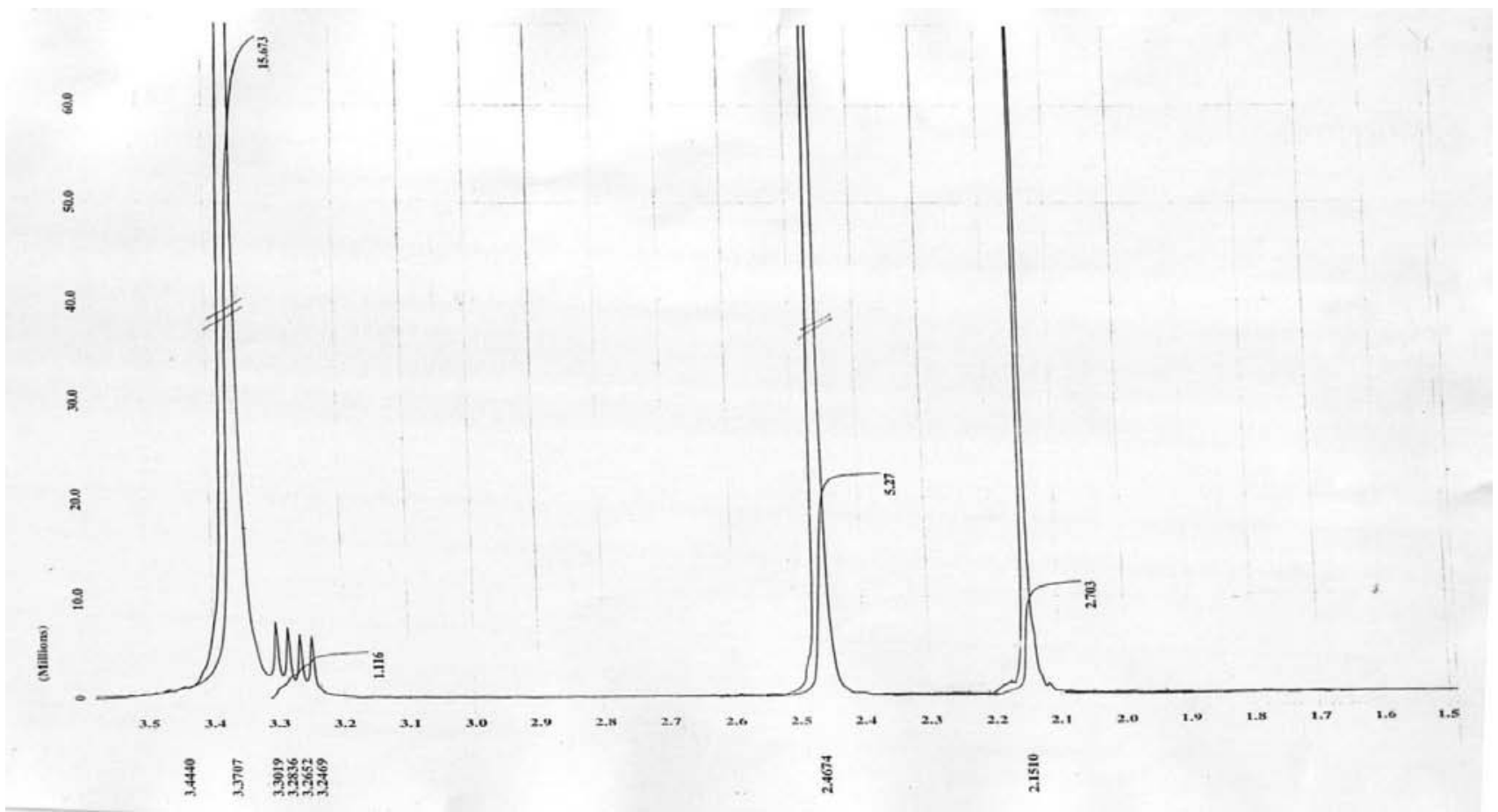

Figure S28. ${ }^{1} \mathrm{H}$ NMR spectrum of compound 11.

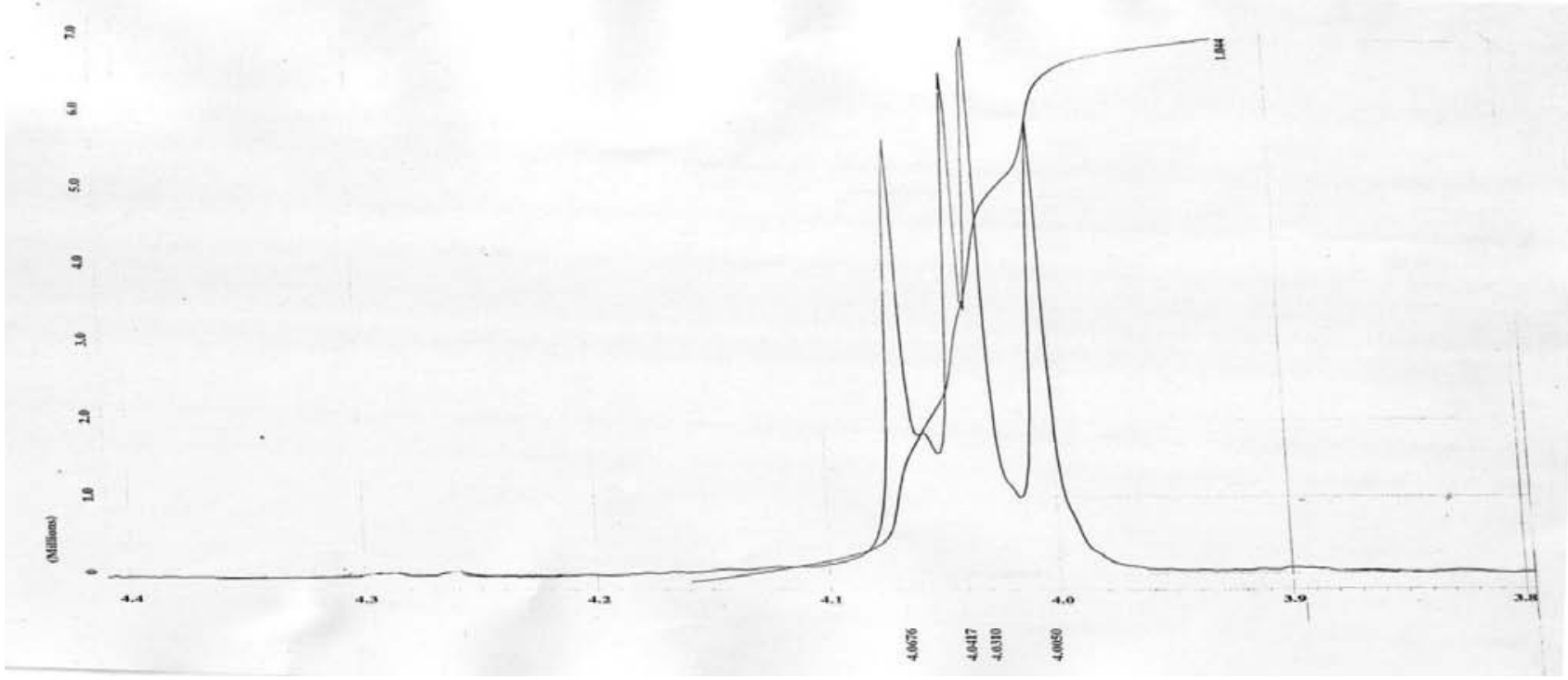

Figure S29. ${ }^{1} \mathrm{H}$ NMR spectrum of compound 11. 


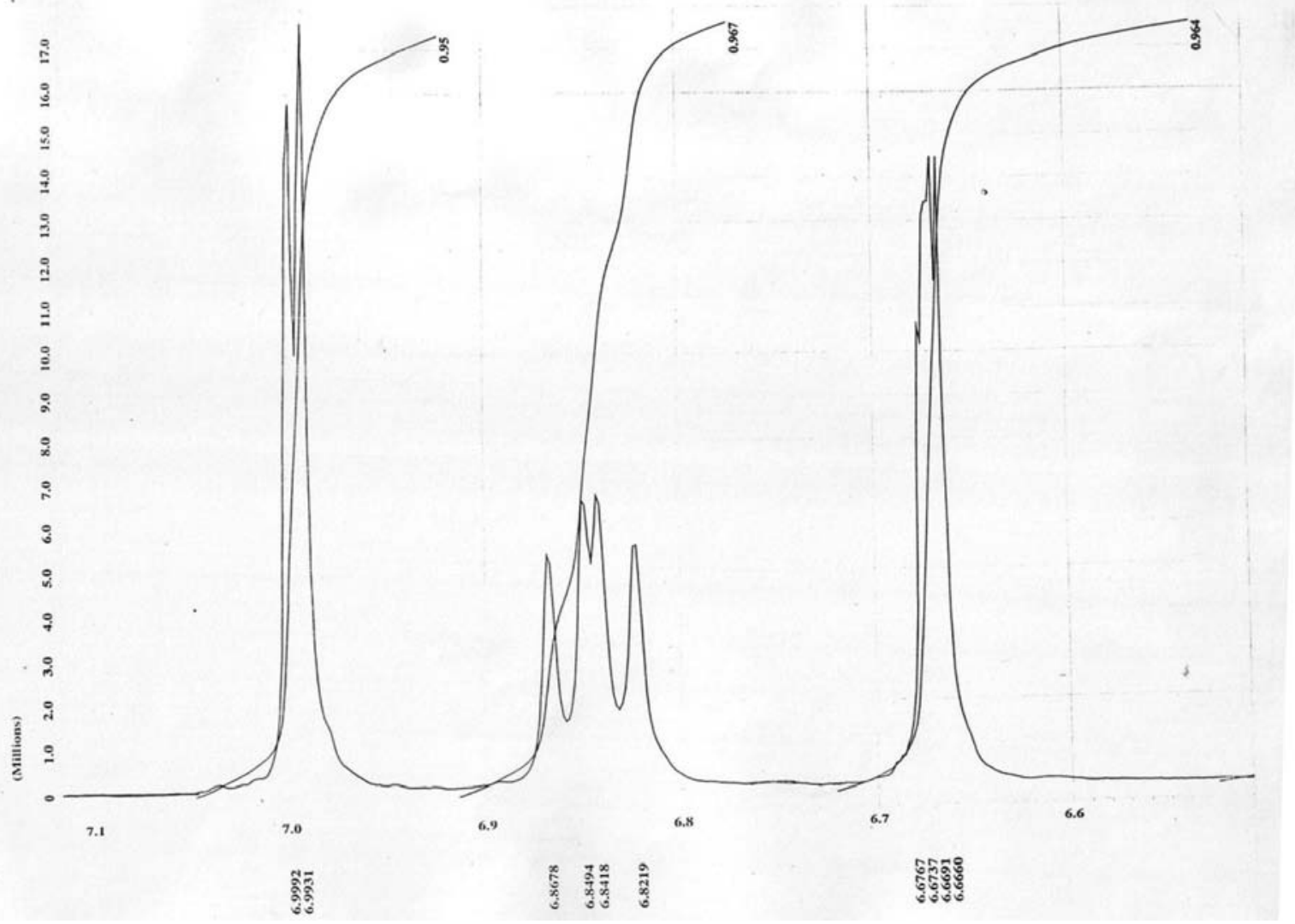

Figure S30. ${ }^{1} \mathrm{H}$ NMR spectrum of compound 11.

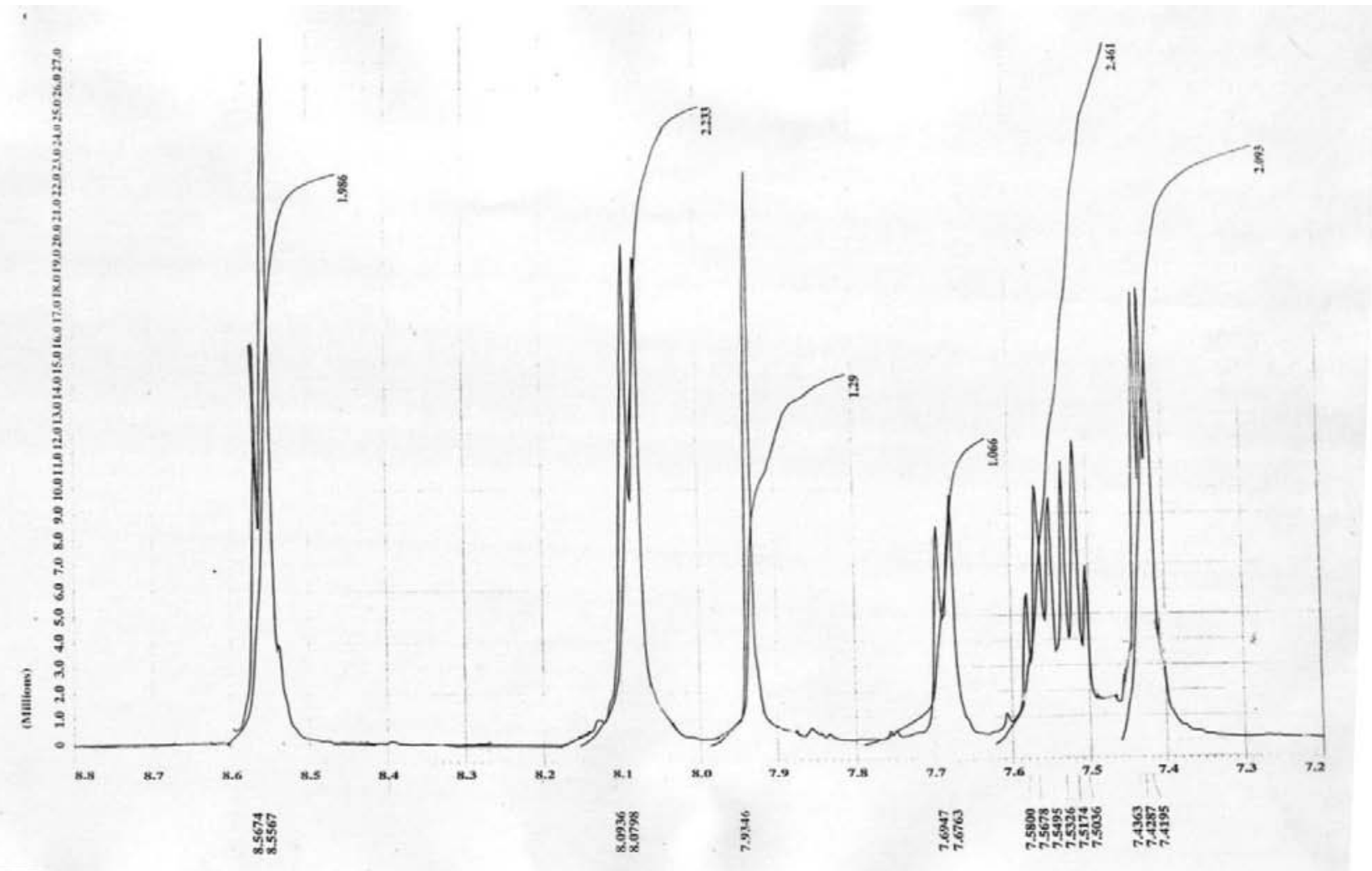

Figure S31. ${ }^{1} \mathrm{H}$ NMR spectrum of compound 11. 


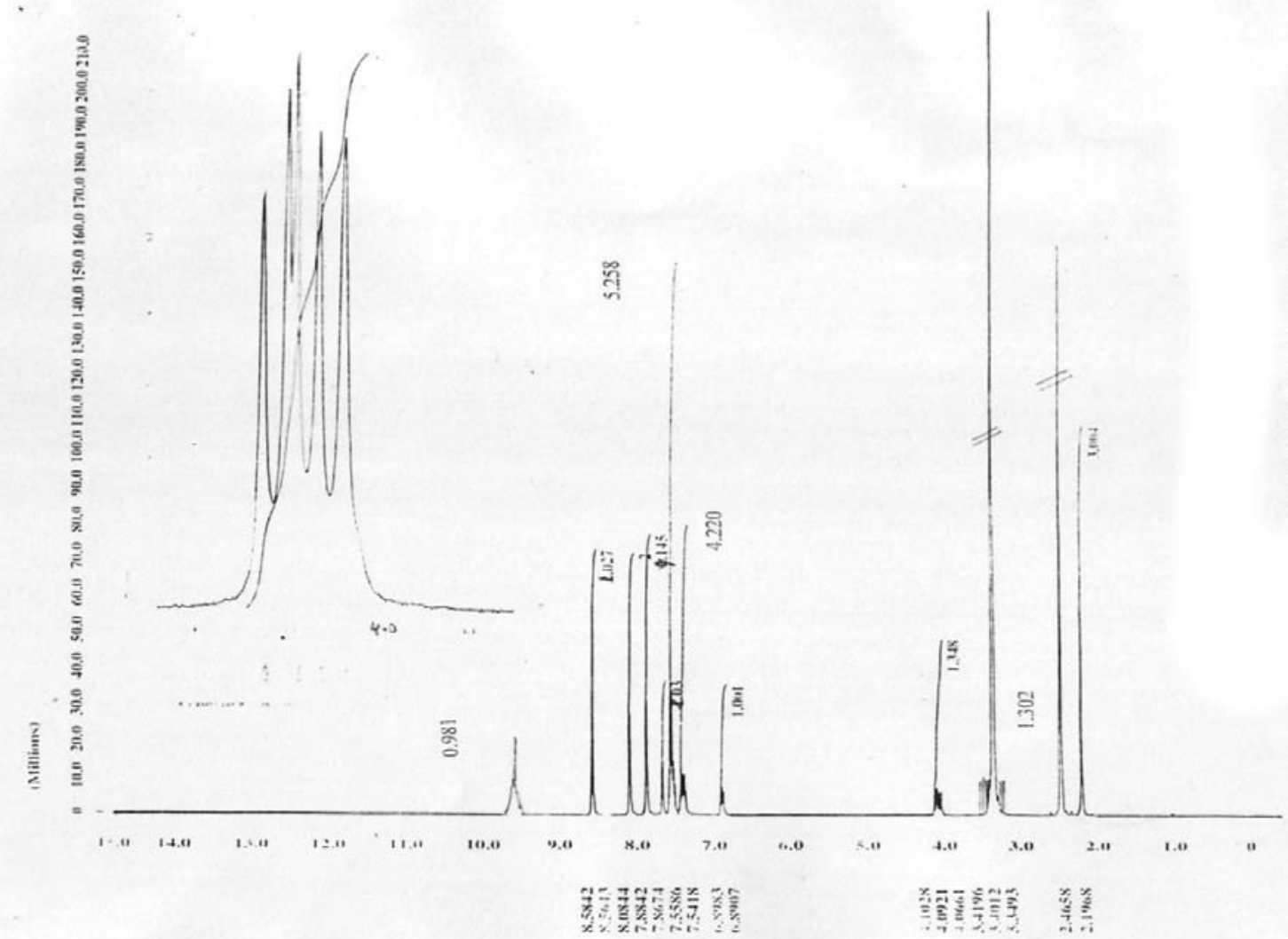

Figure S32. ${ }^{1} \mathrm{H}$ NMR spectrum of compound 14.

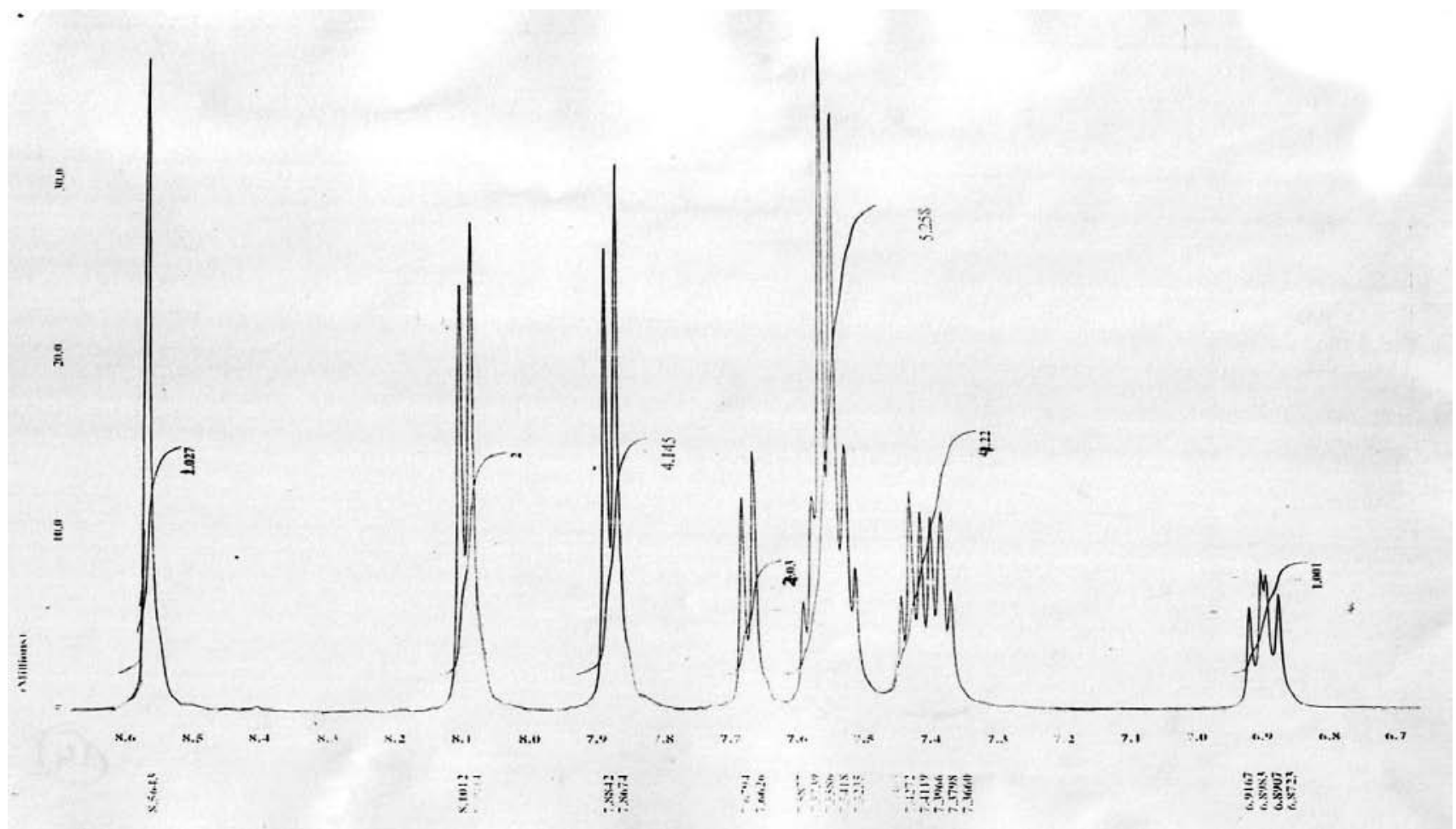

Figure S33. ${ }^{1} \mathrm{H}$ NMR spectrum of compound 14. 


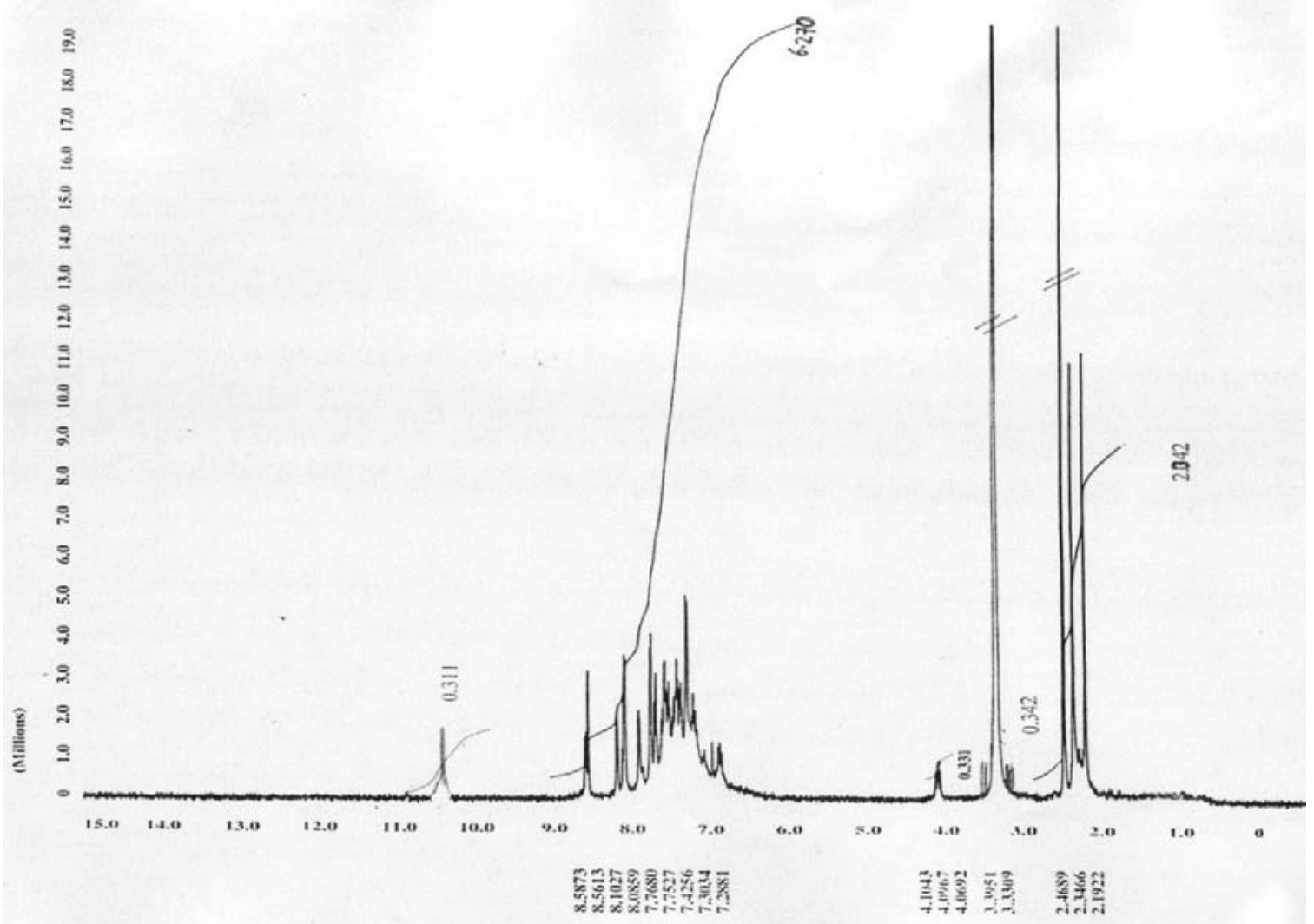

Figure S34. ${ }^{1} \mathrm{H}$ NMR spectrum of compound $\mathbf{1 5}$.

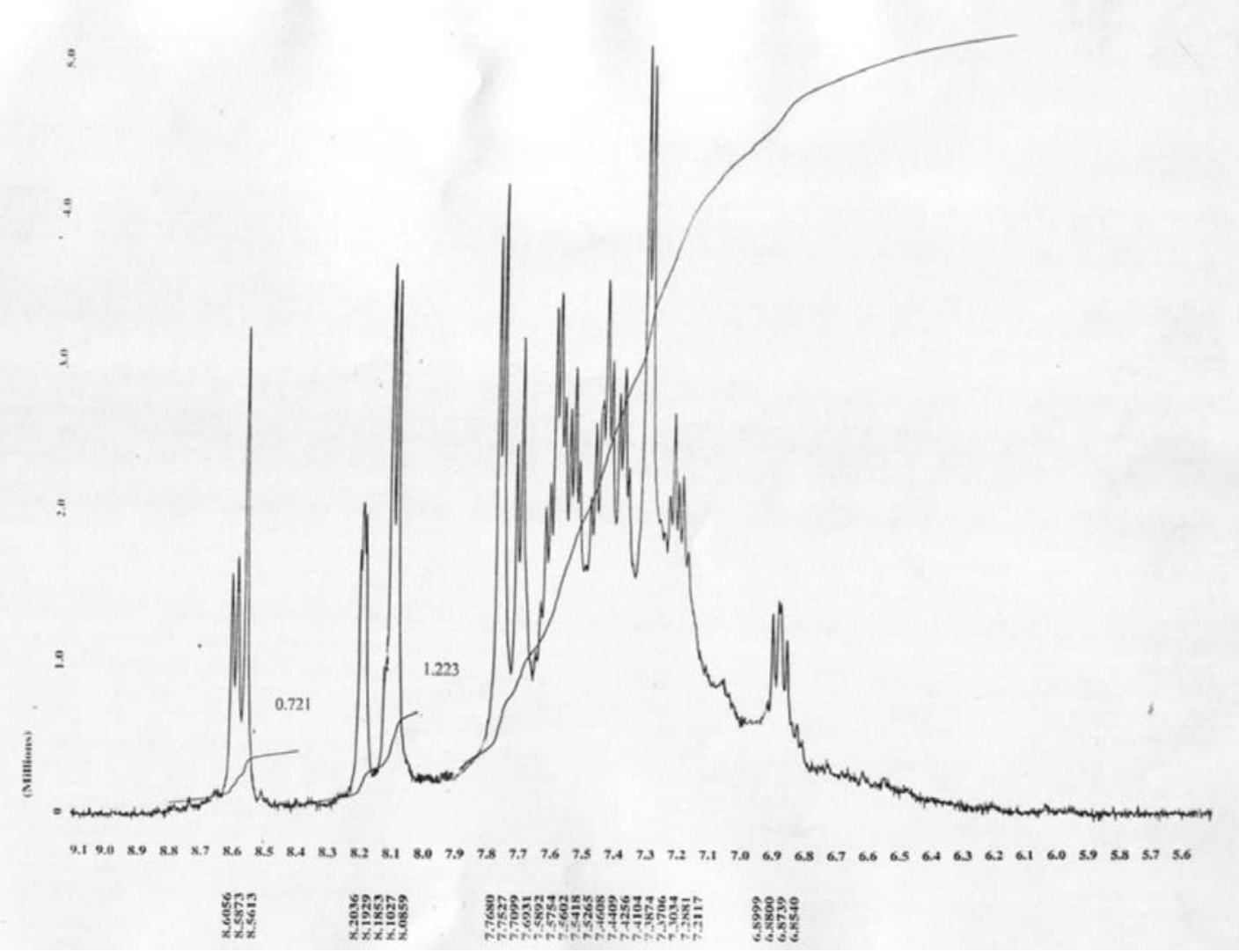

Figure S35. ${ }^{1} \mathrm{H}$ NMR spectrum of compound 15. 


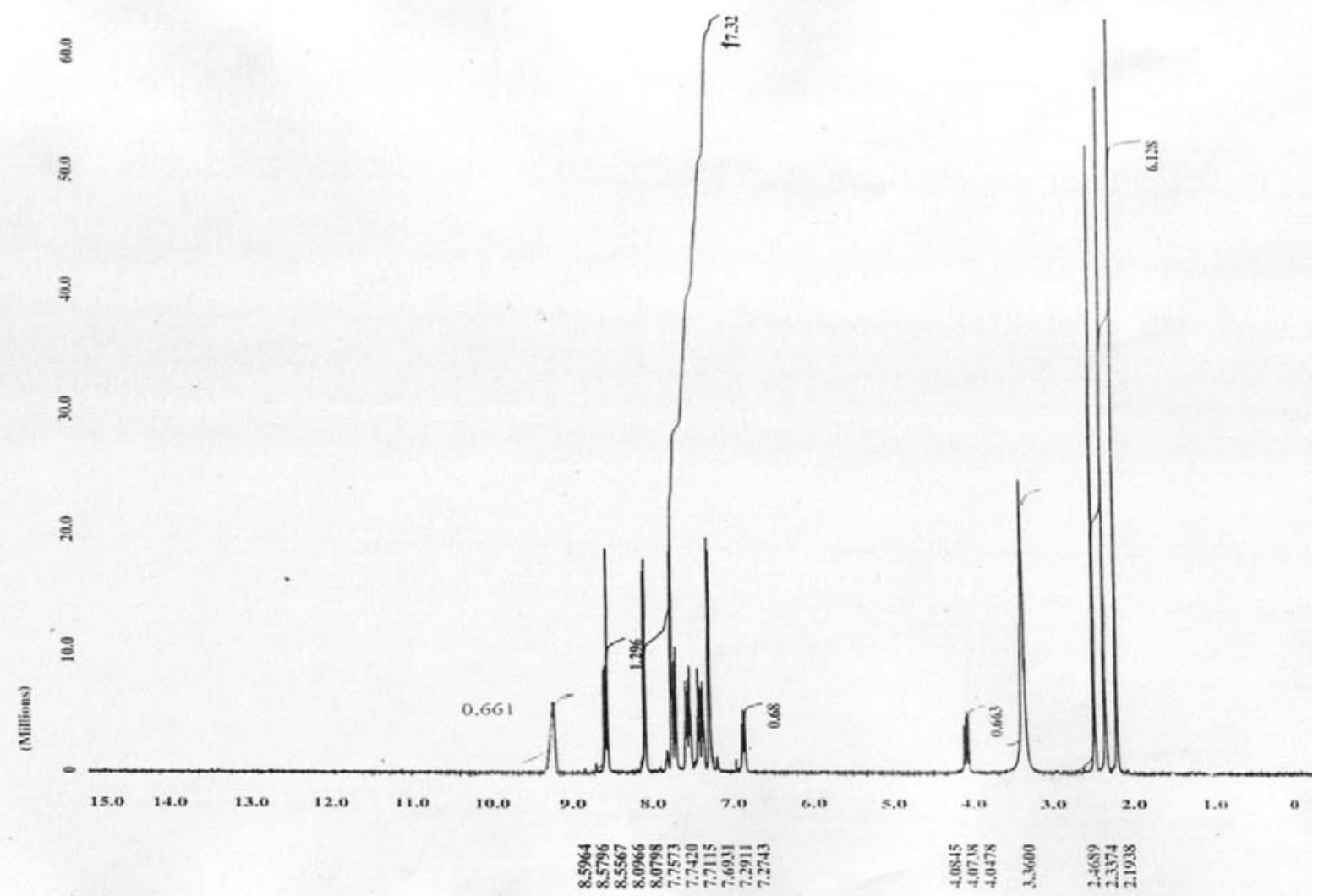

Figure S36. ${ }^{1} \mathrm{H}$ NMR spectrum of compound 17.

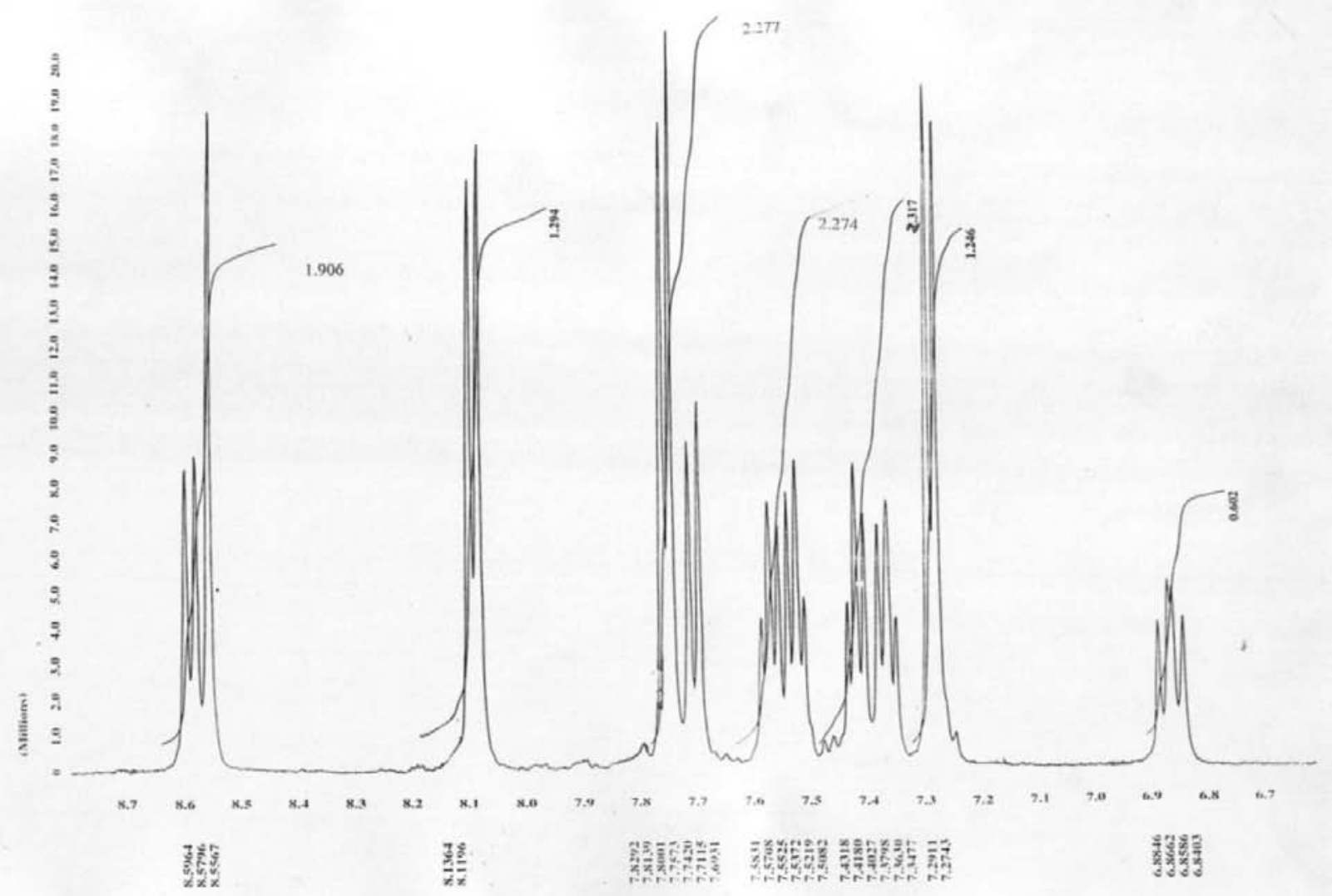

Figure S37. ${ }^{1} \mathrm{H}$ NMR spectrum of compound 17. 


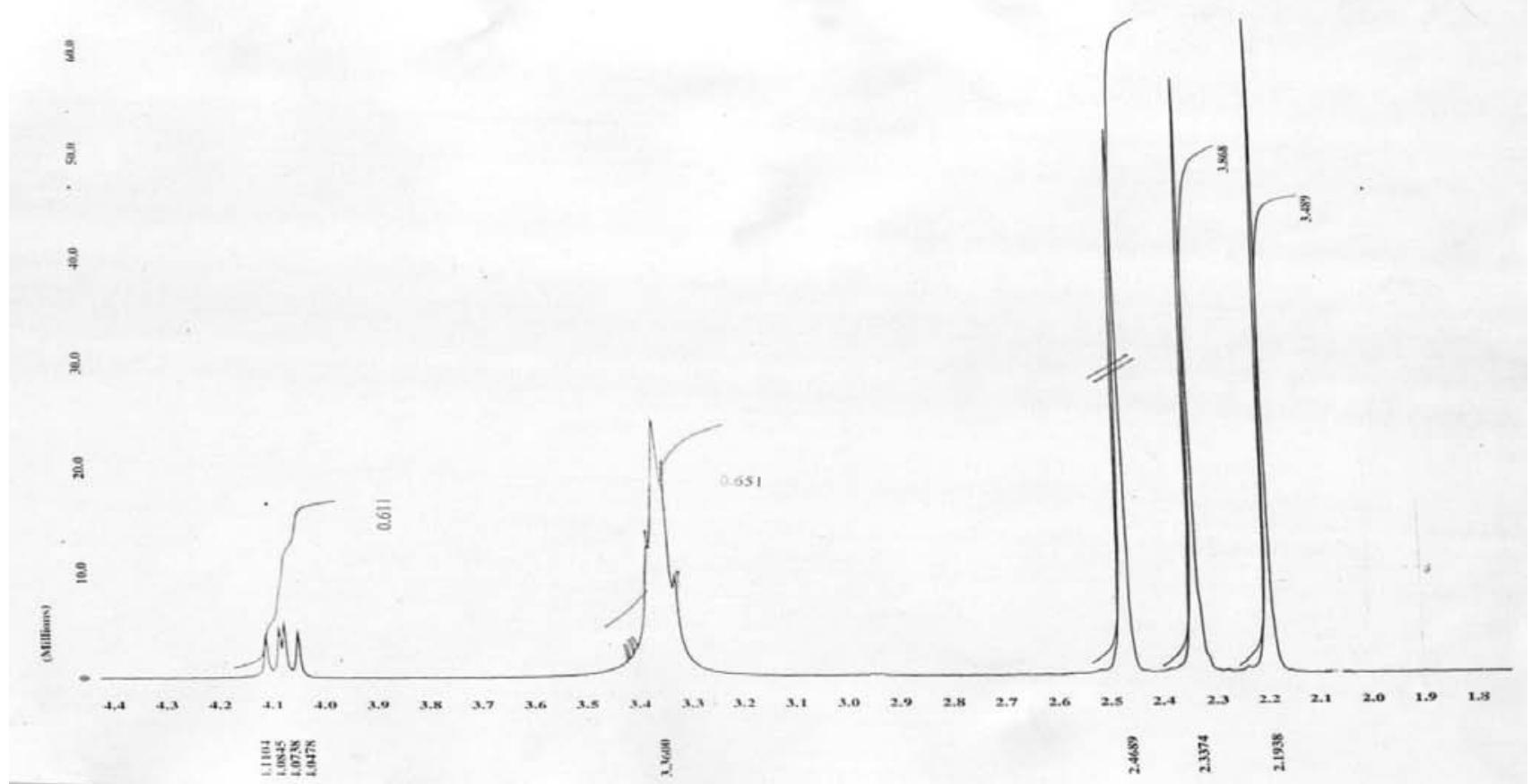

Figure S38. ${ }^{1} \mathrm{H}$ NMR spectrum of compound 17.

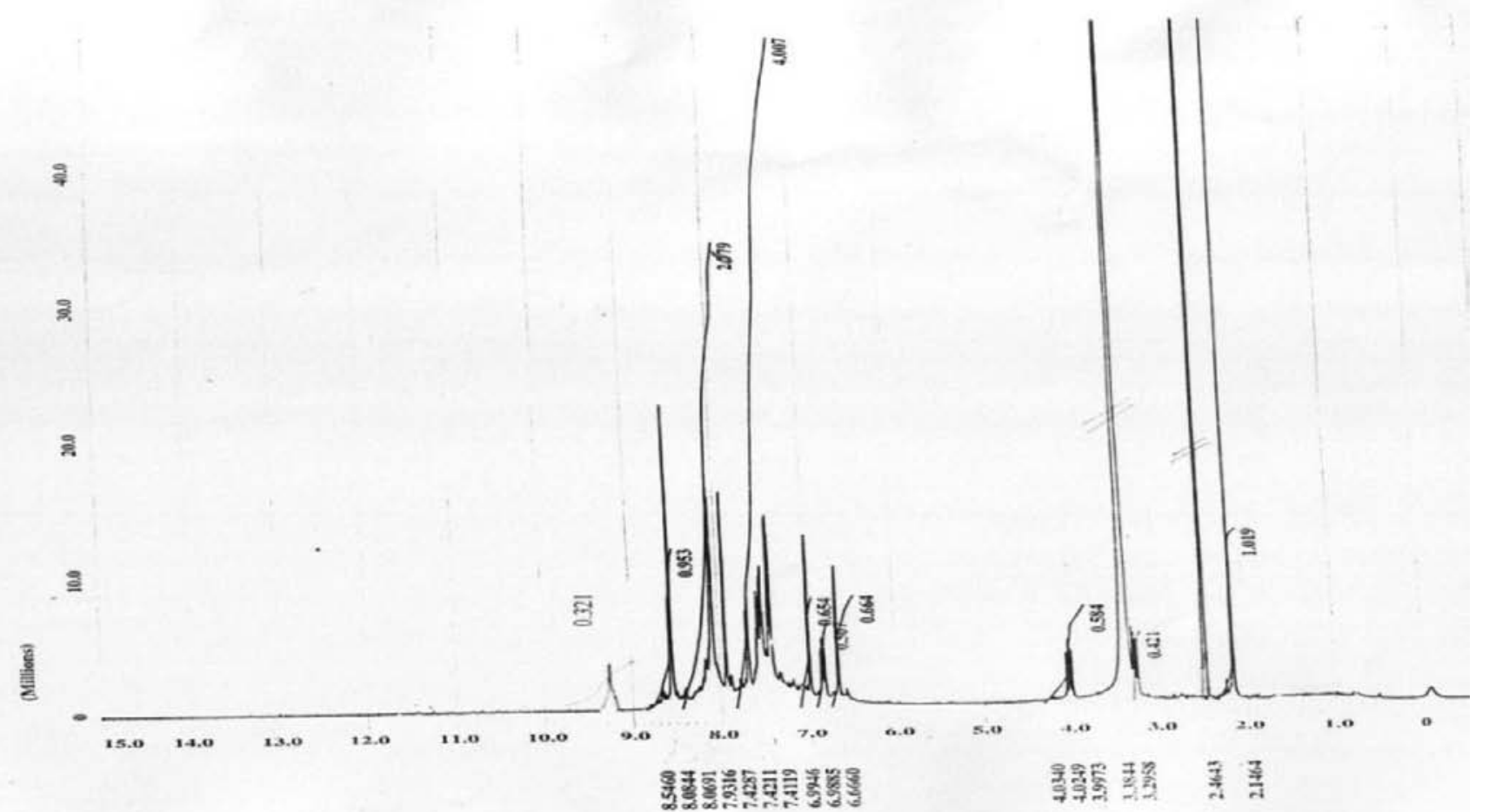

Figure S39. ${ }^{1} \mathrm{H}$ NMR spectrum of compound 18. 


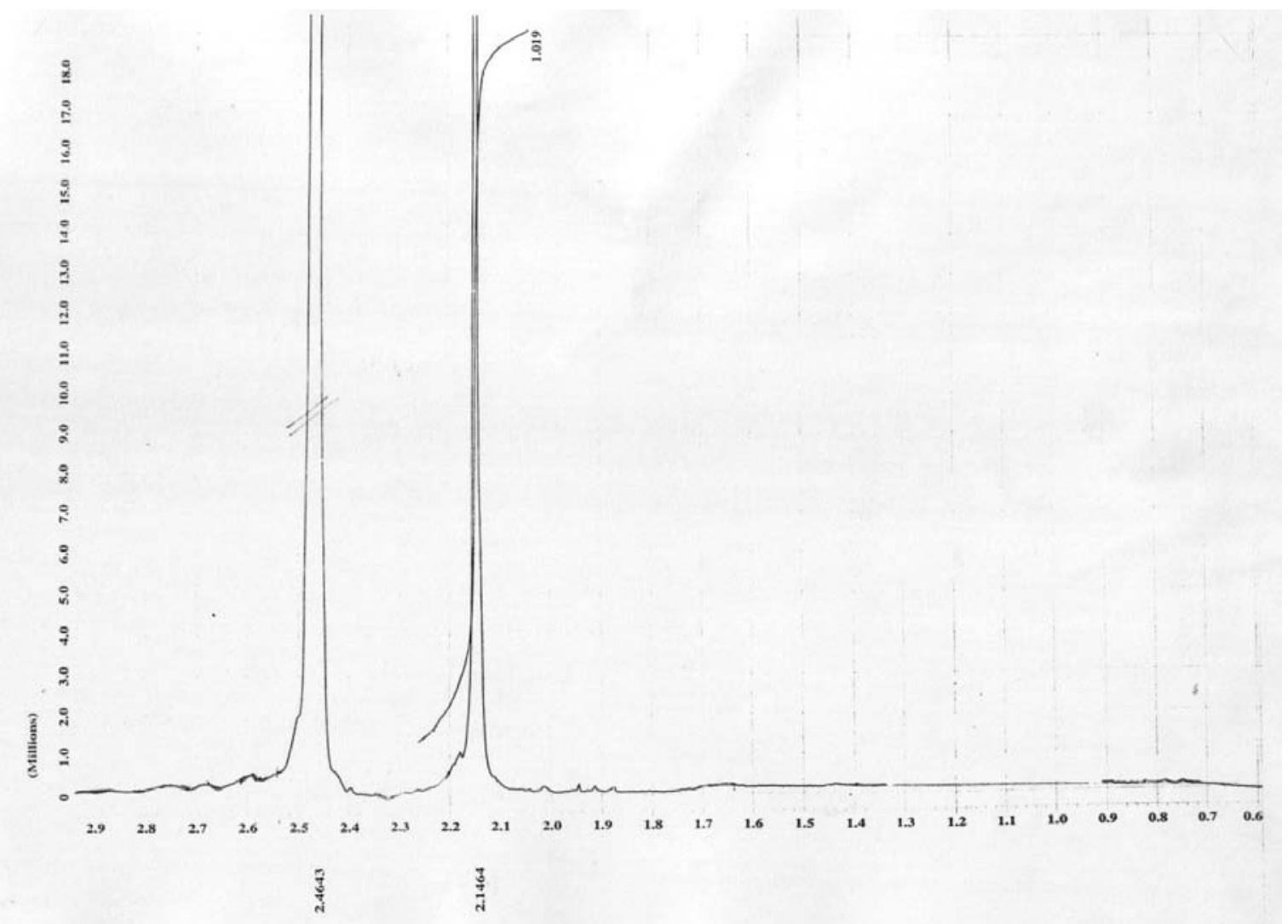

Figure S40. ${ }^{1} \mathrm{H}$ NMR spectrum of compound $\mathbf{1 8}$.

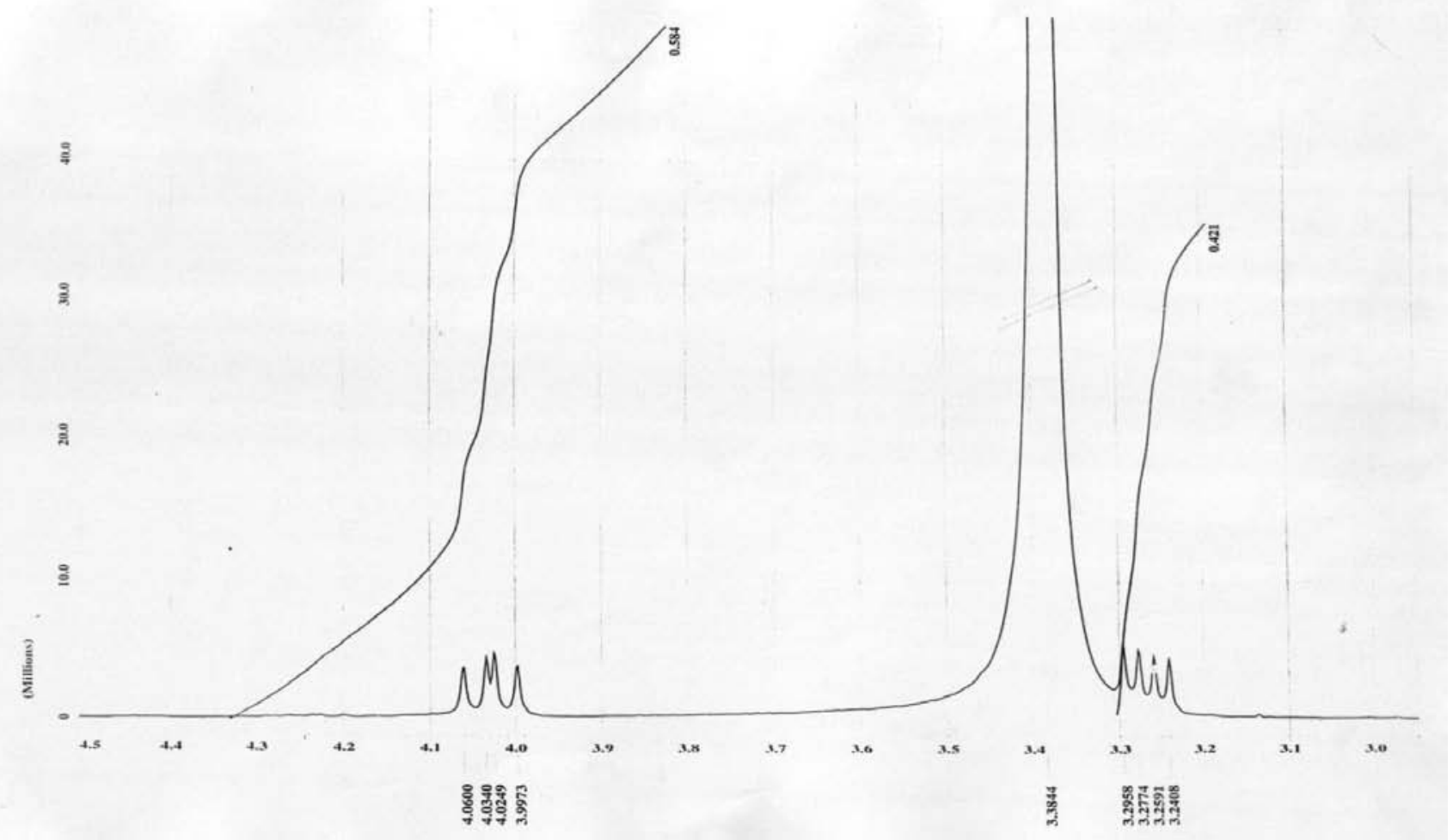

Figure S41. ${ }^{1} \mathrm{H}$ NMR spectrum of compound 18. 


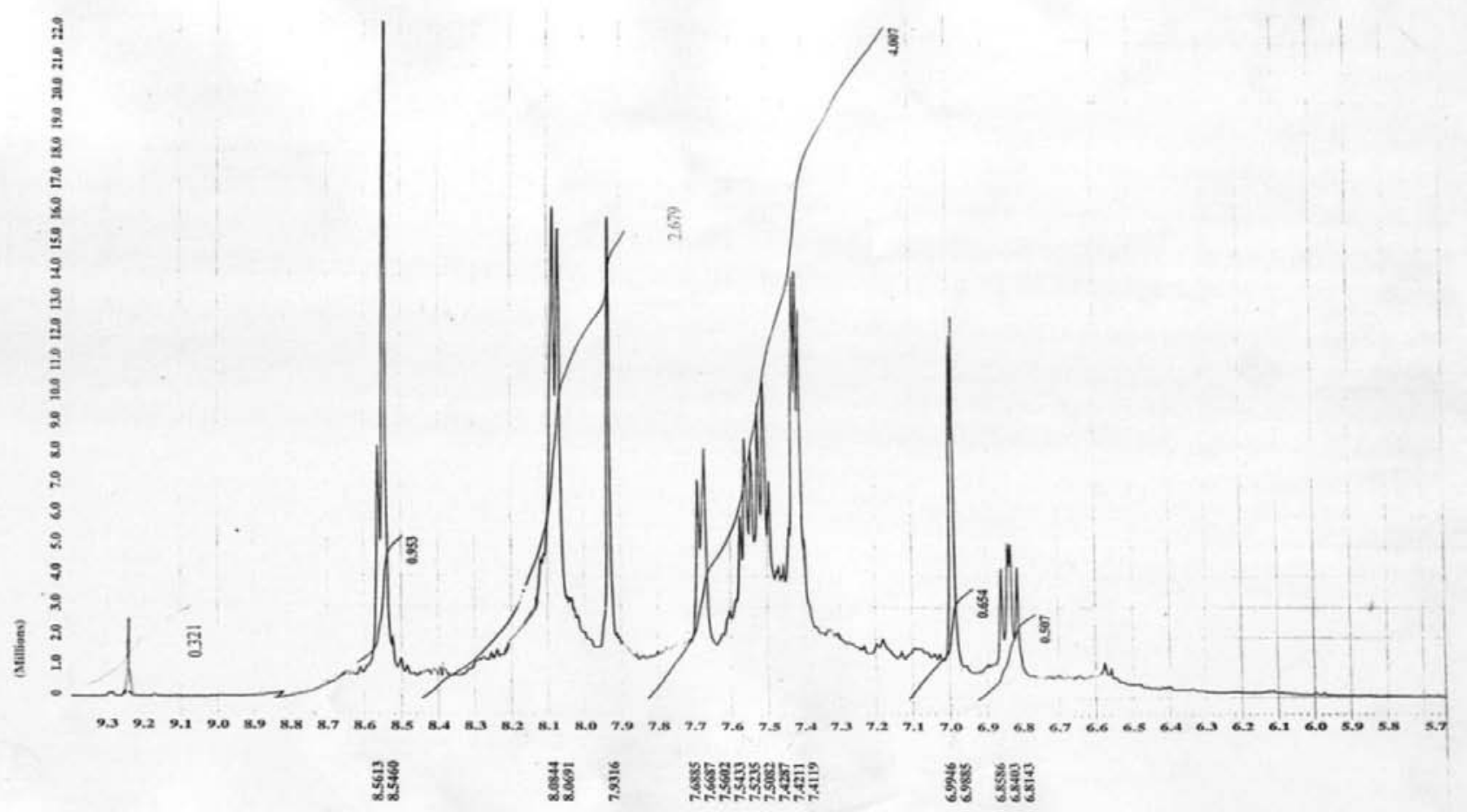

Figure S42. ${ }^{1} \mathrm{H}$ NMR spectrum of compound 18.

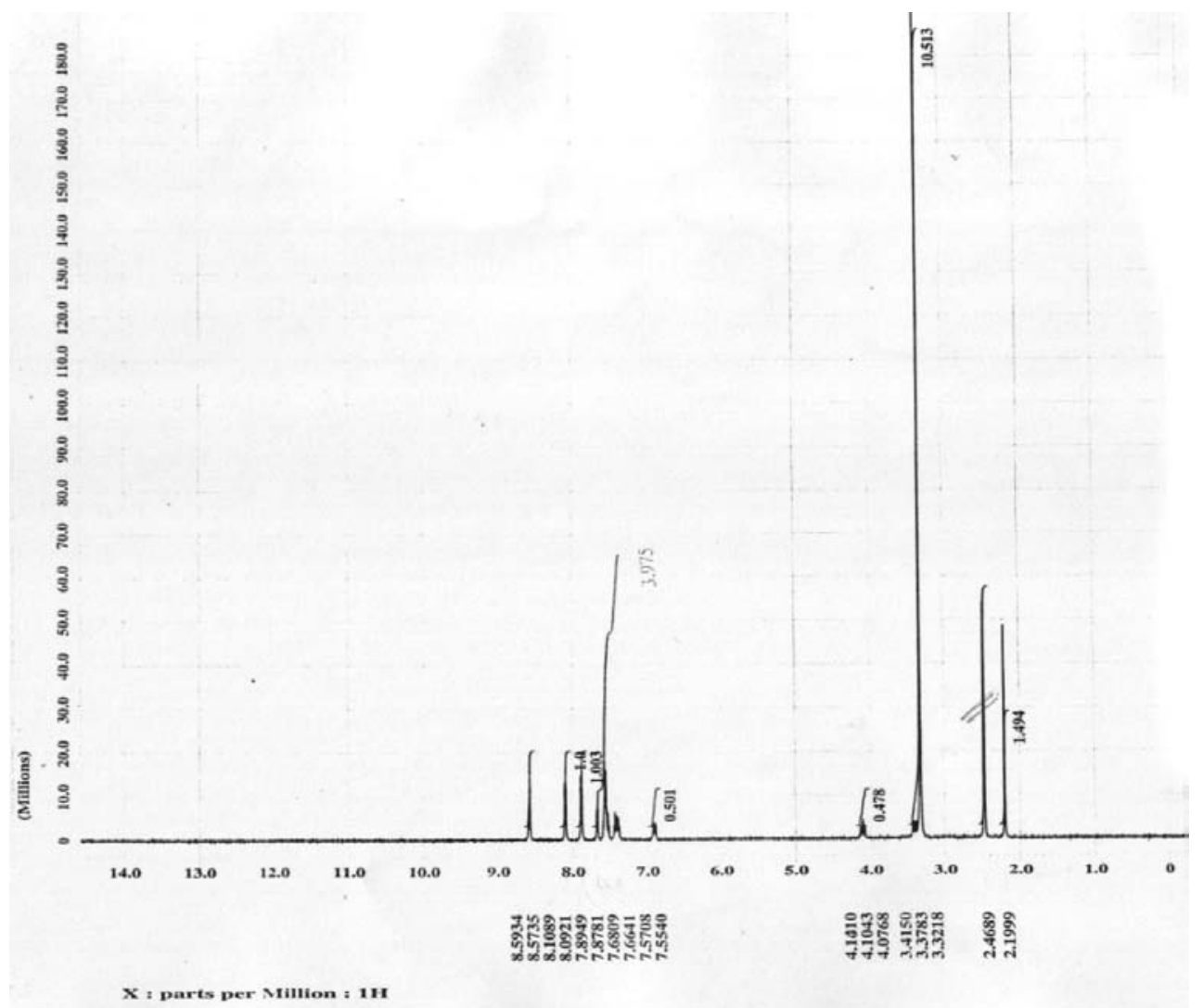

Figure S43. ${ }^{1} \mathrm{H}$ NMR spectrum of compound 19. 


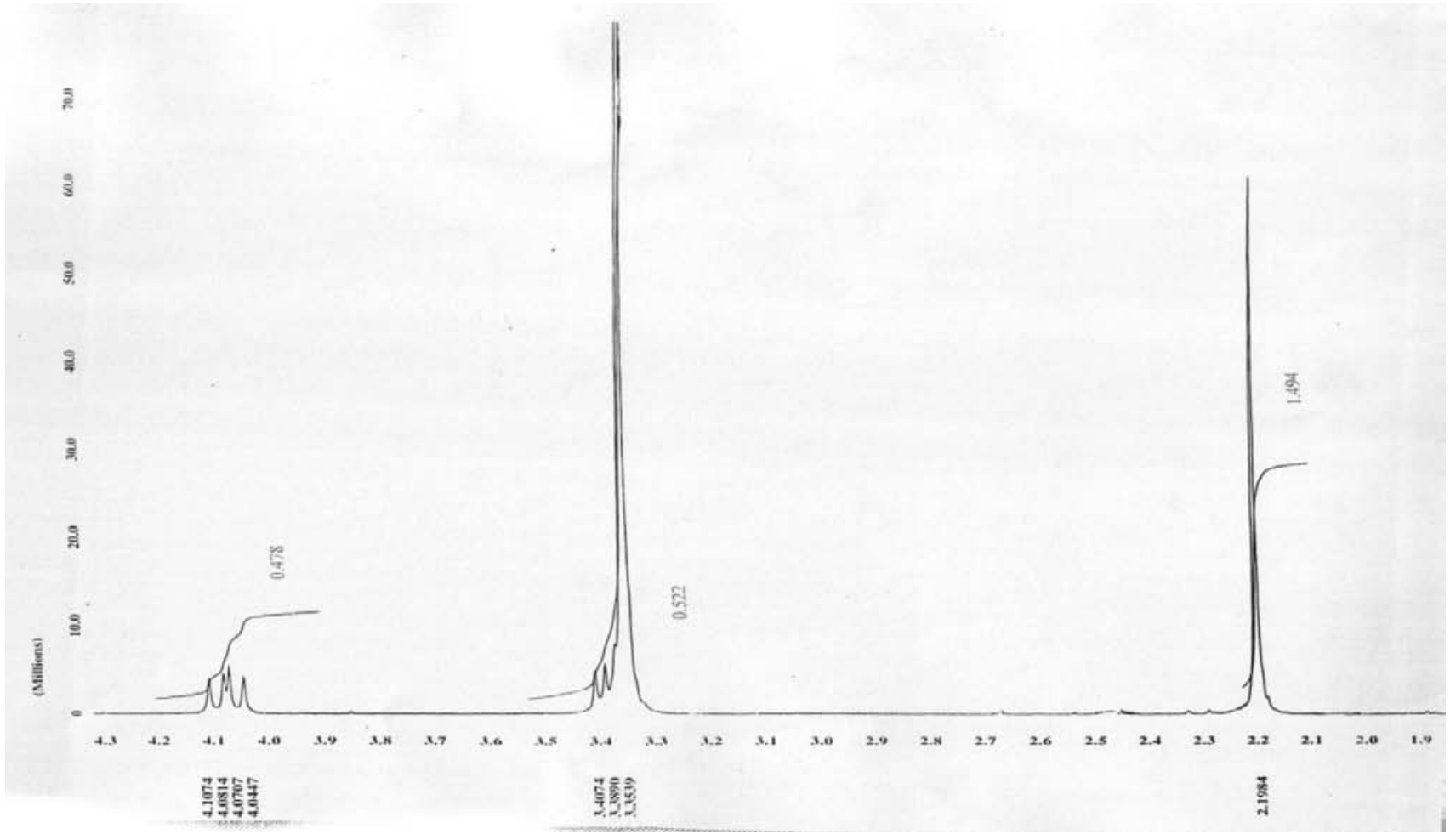

Figure S44. ${ }^{1} \mathrm{H}$ NMR spectrum of compound 19.

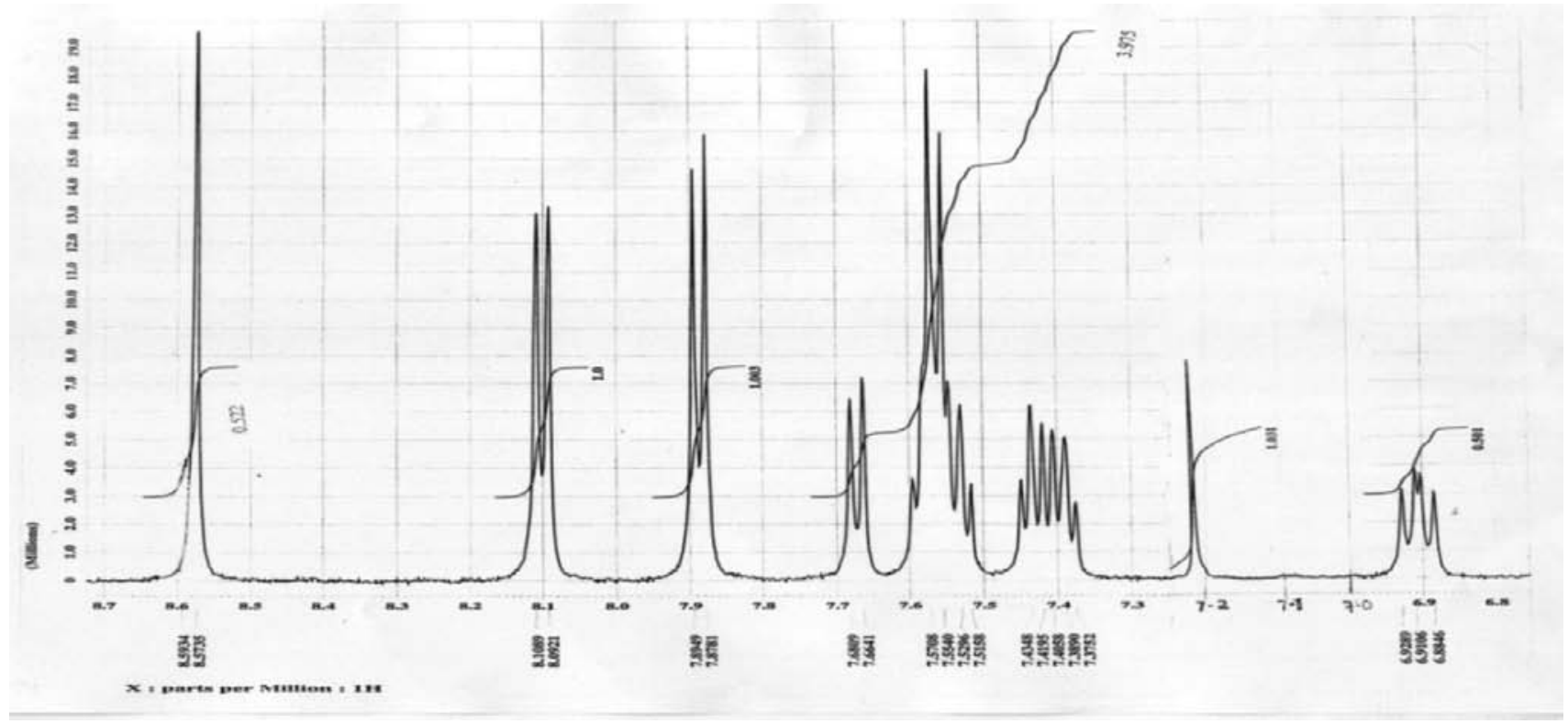

Figure S45. ${ }^{1} \mathrm{H}$ NMR spectrum of compound 19. 


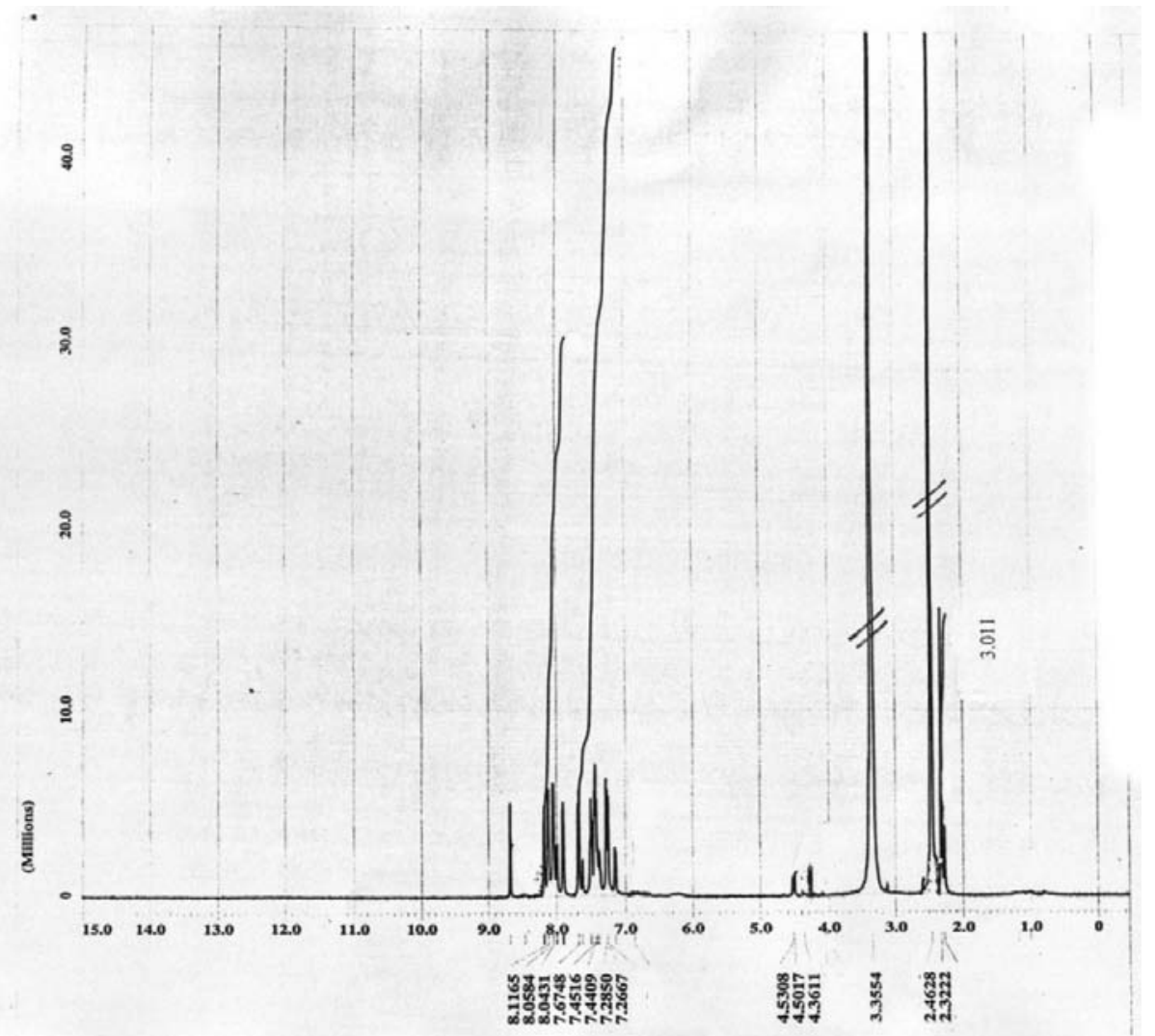

Figure S46. ${ }^{1} \mathrm{H}$ NMR spectrum of compound 20.

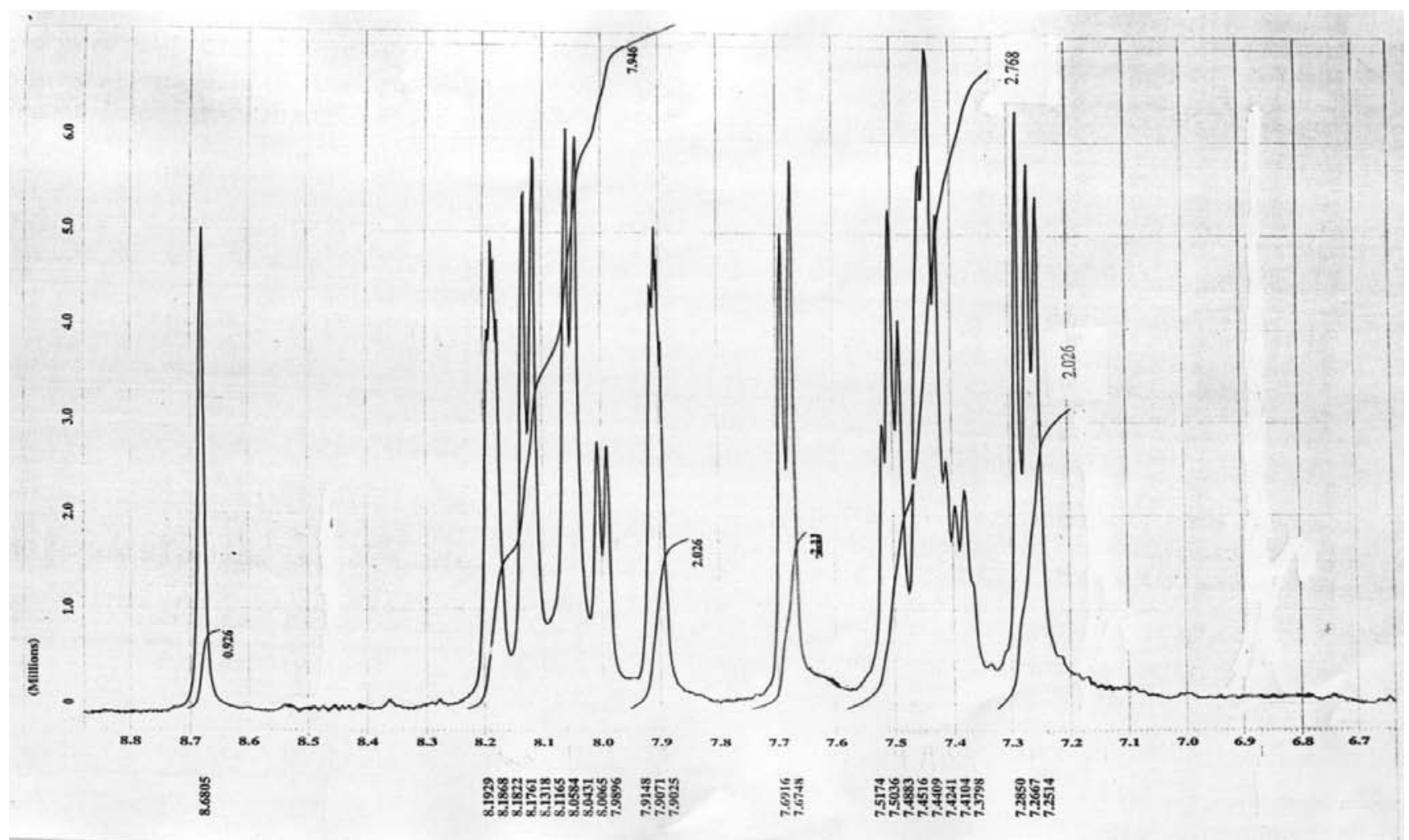

Figure S47. ${ }^{1} \mathrm{H}$ NMR spectrum of compound 20. 
Vol. 22, No. 7, 2011

Masan

S25

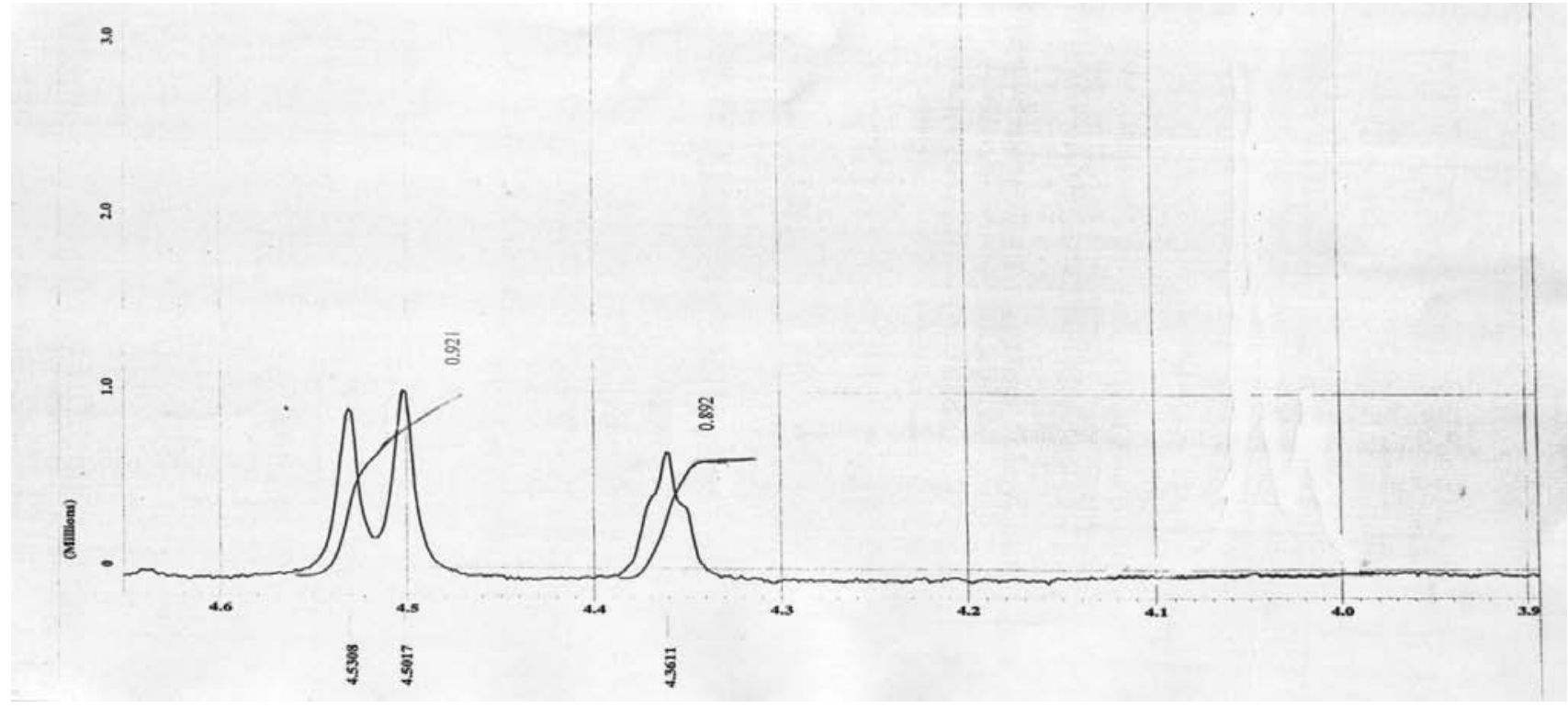

Figure S48. ${ }^{1} \mathrm{H}$ NMR spectrum of compound 20

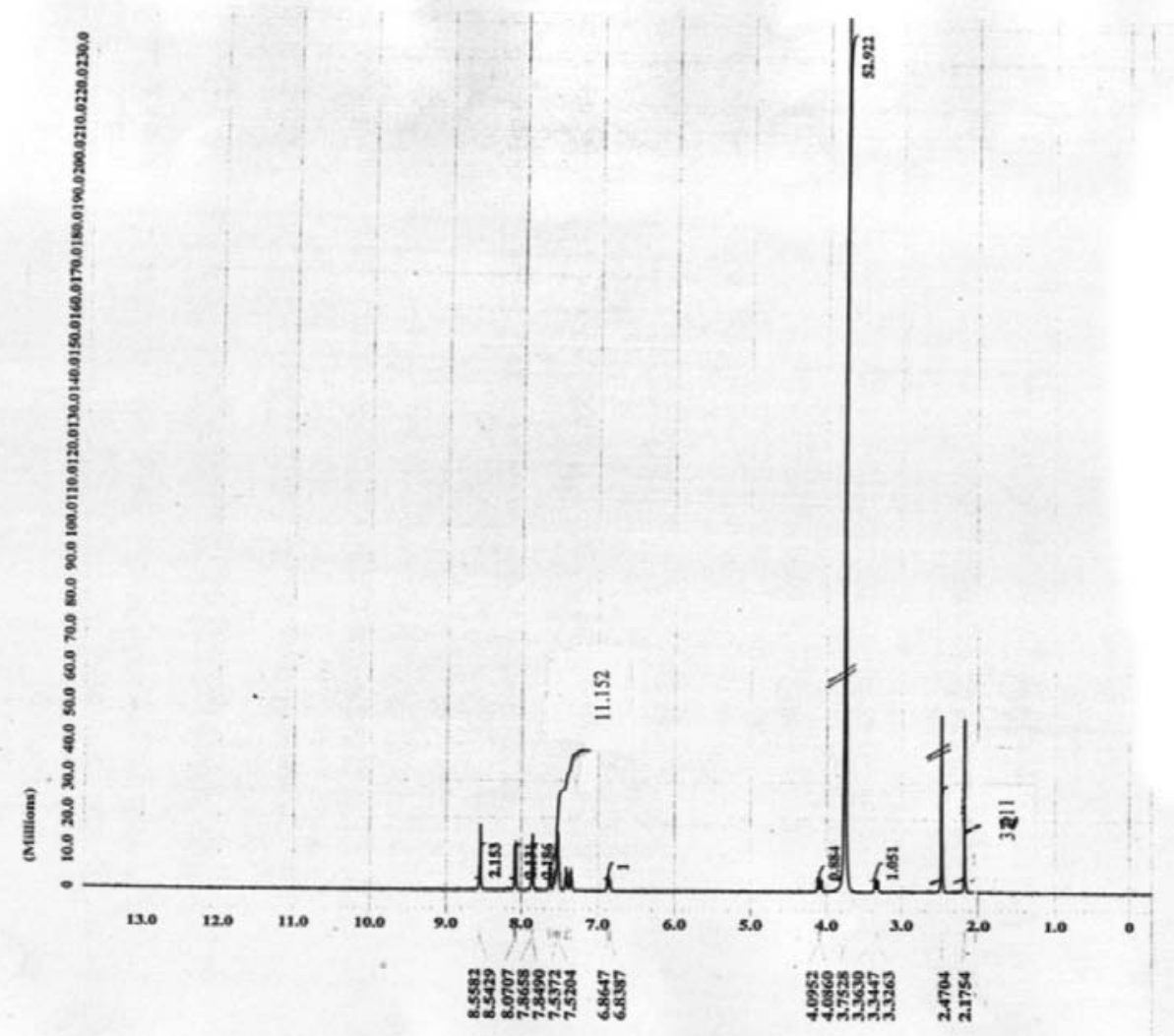

Figure S49. ${ }^{1} \mathrm{H}$ NMR spectrum of compound 22. 


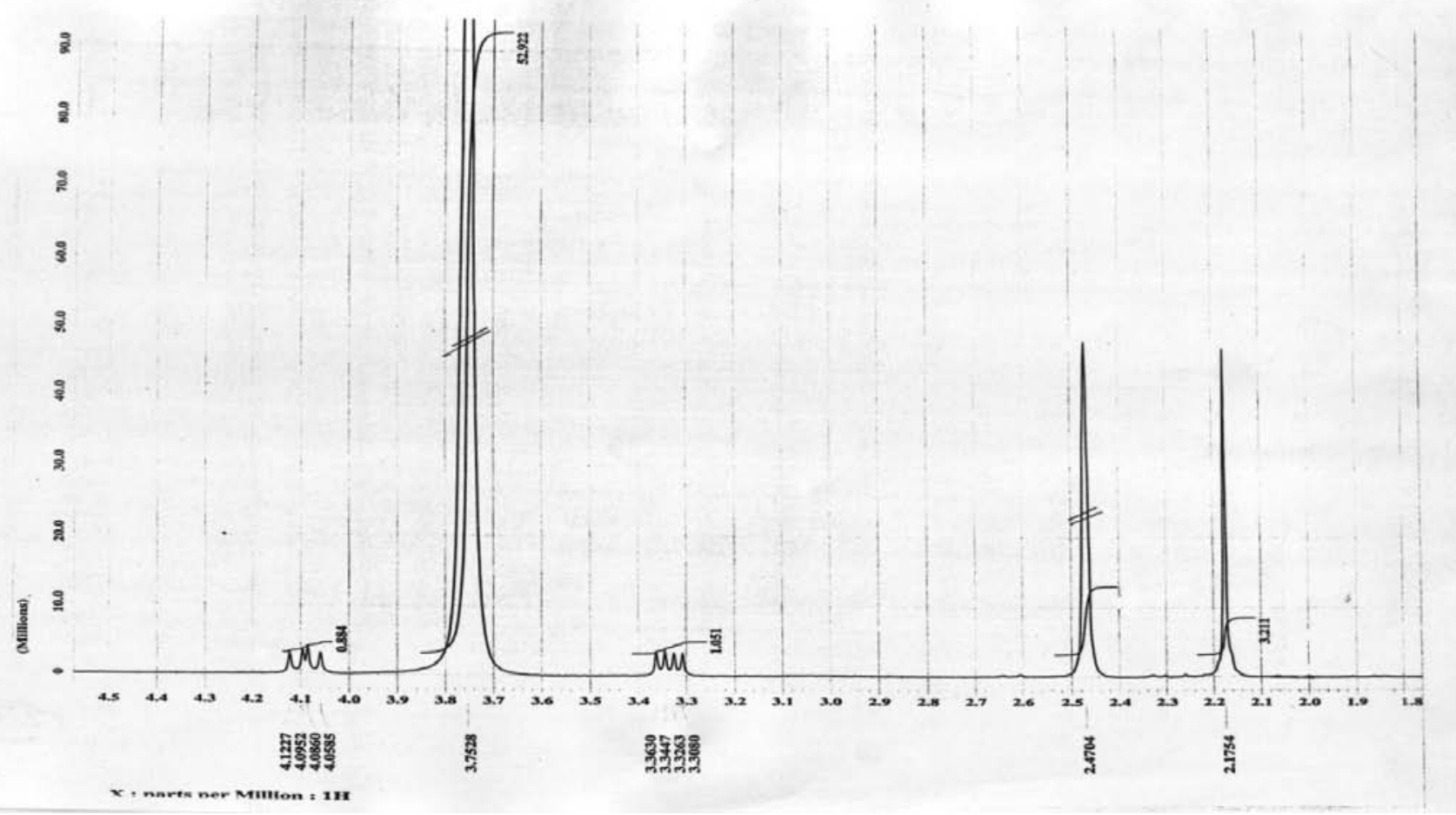

Figure S50. ${ }^{1} \mathrm{H}$ NMR spectrum of compound 22.

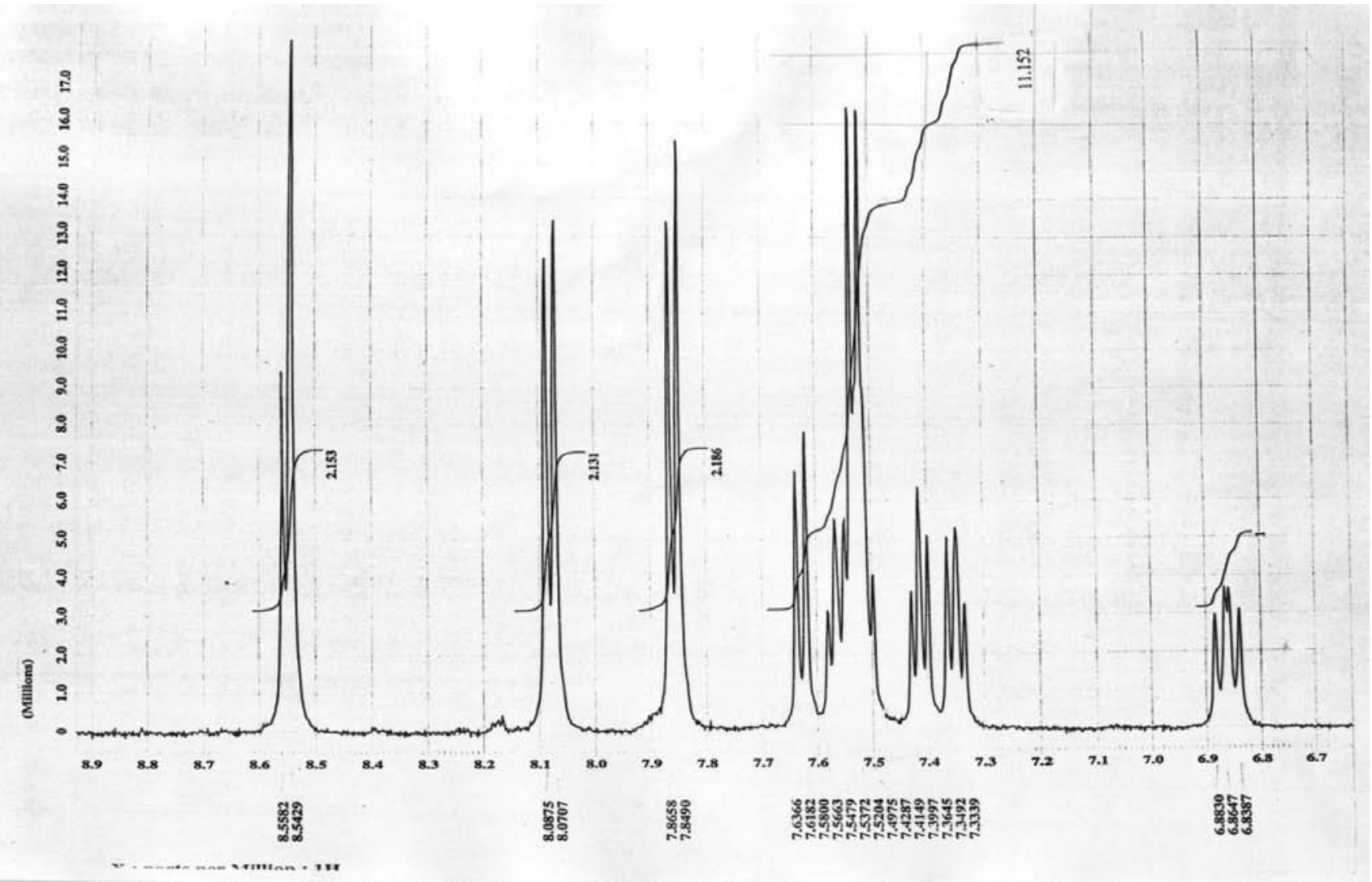

Figure S51. ${ }^{1} \mathrm{H}$ NMR spectrum of compound 22. 


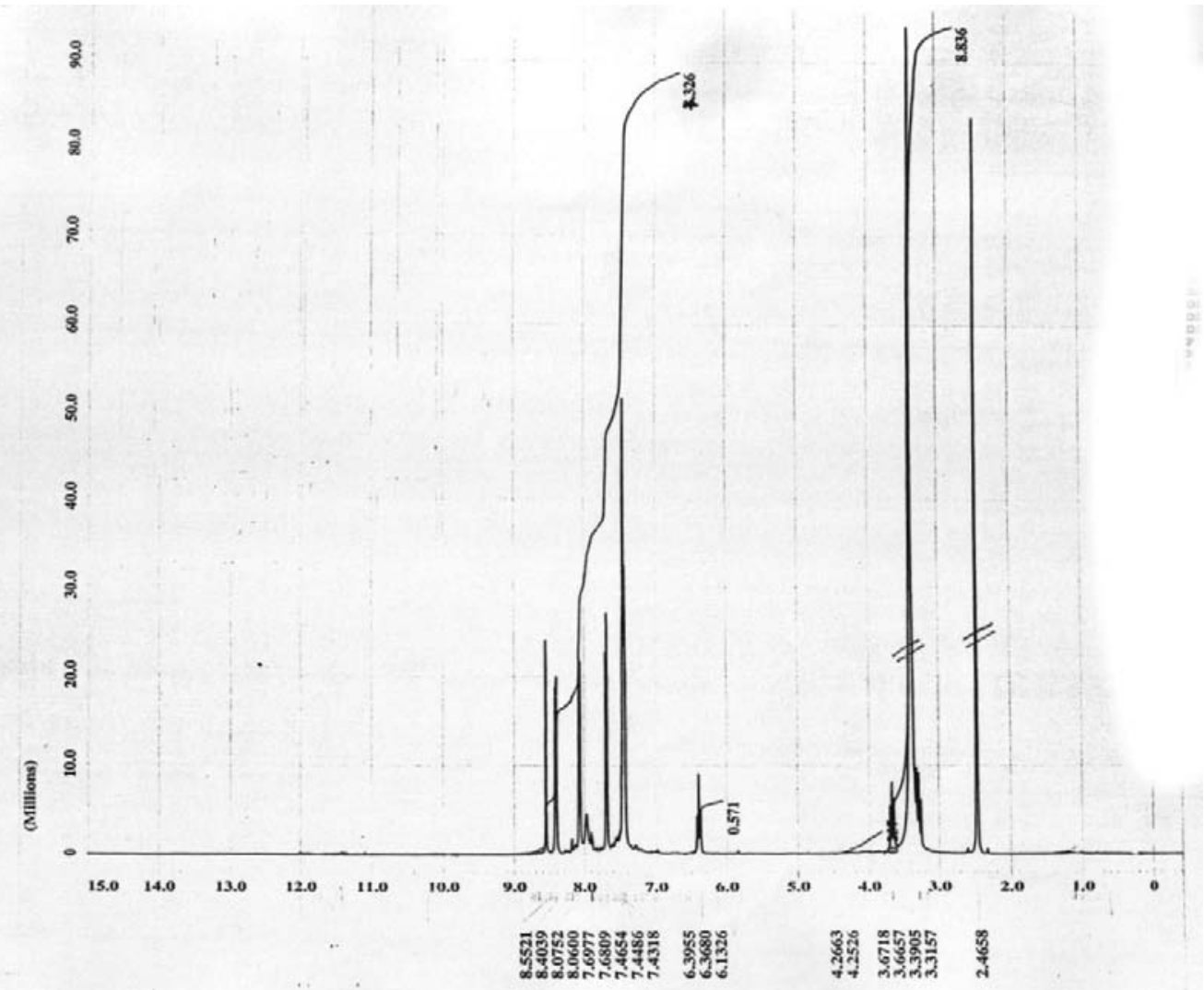

Figure S52. ${ }^{1} \mathrm{H}$ NMR spectrum of compound 26.

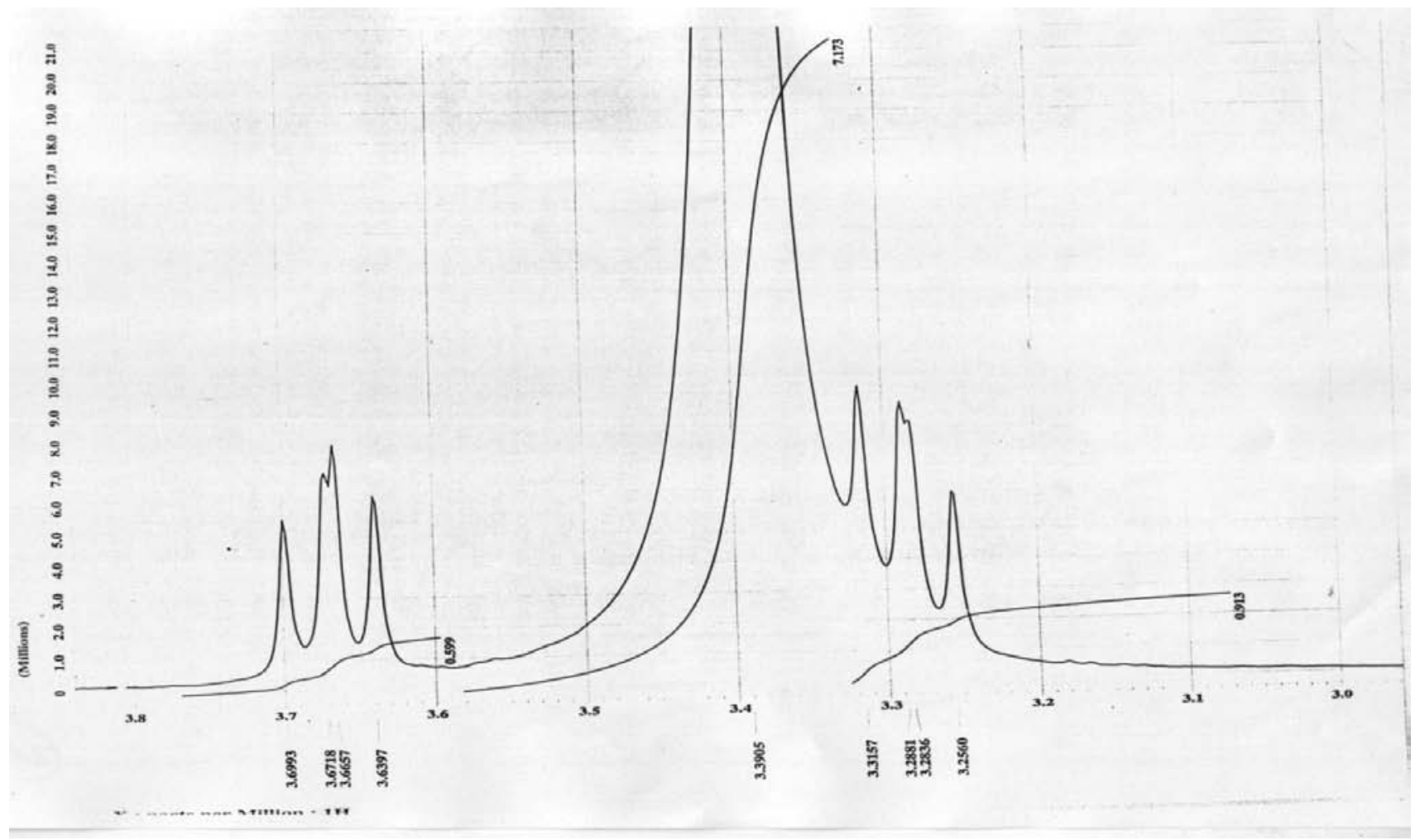

Figure S53. ${ }^{1} \mathrm{H}$ NMR spectrum of compound 26. 


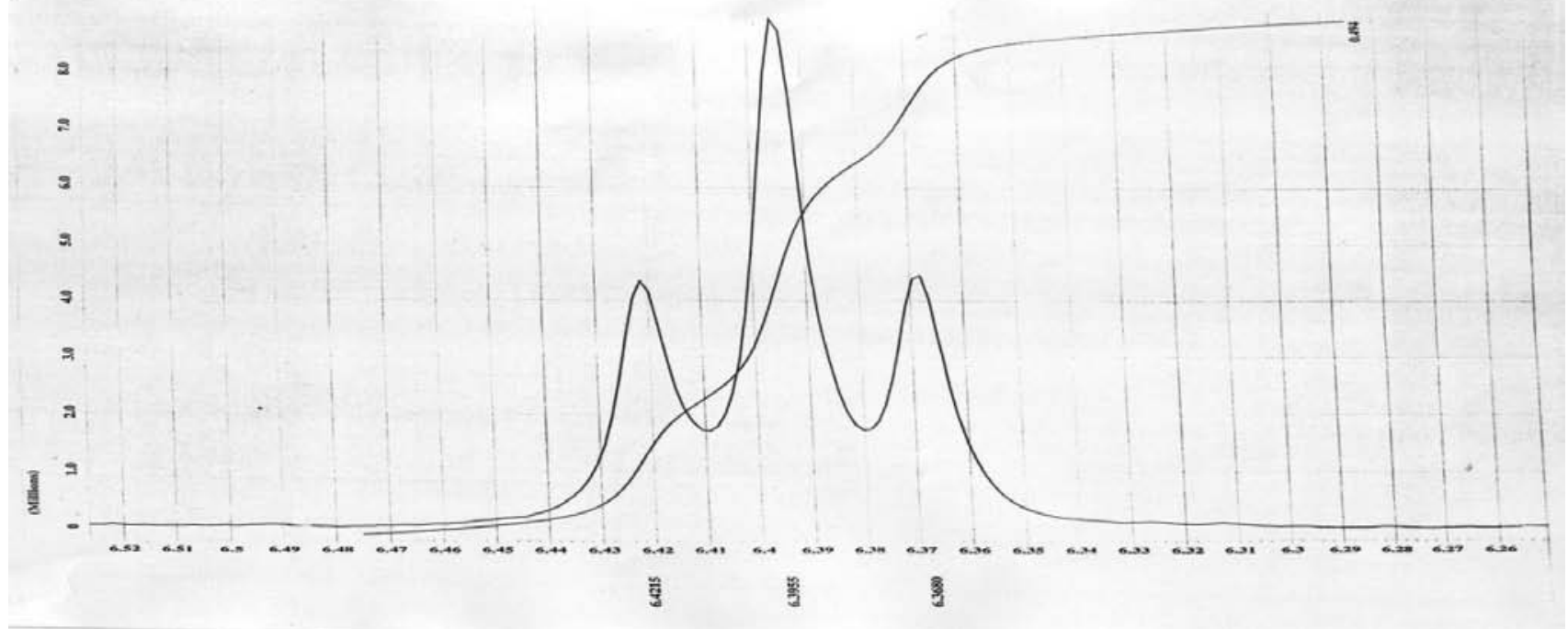

Figure S54. ${ }^{1} \mathrm{H}$ NMR spectrum of compound 26.

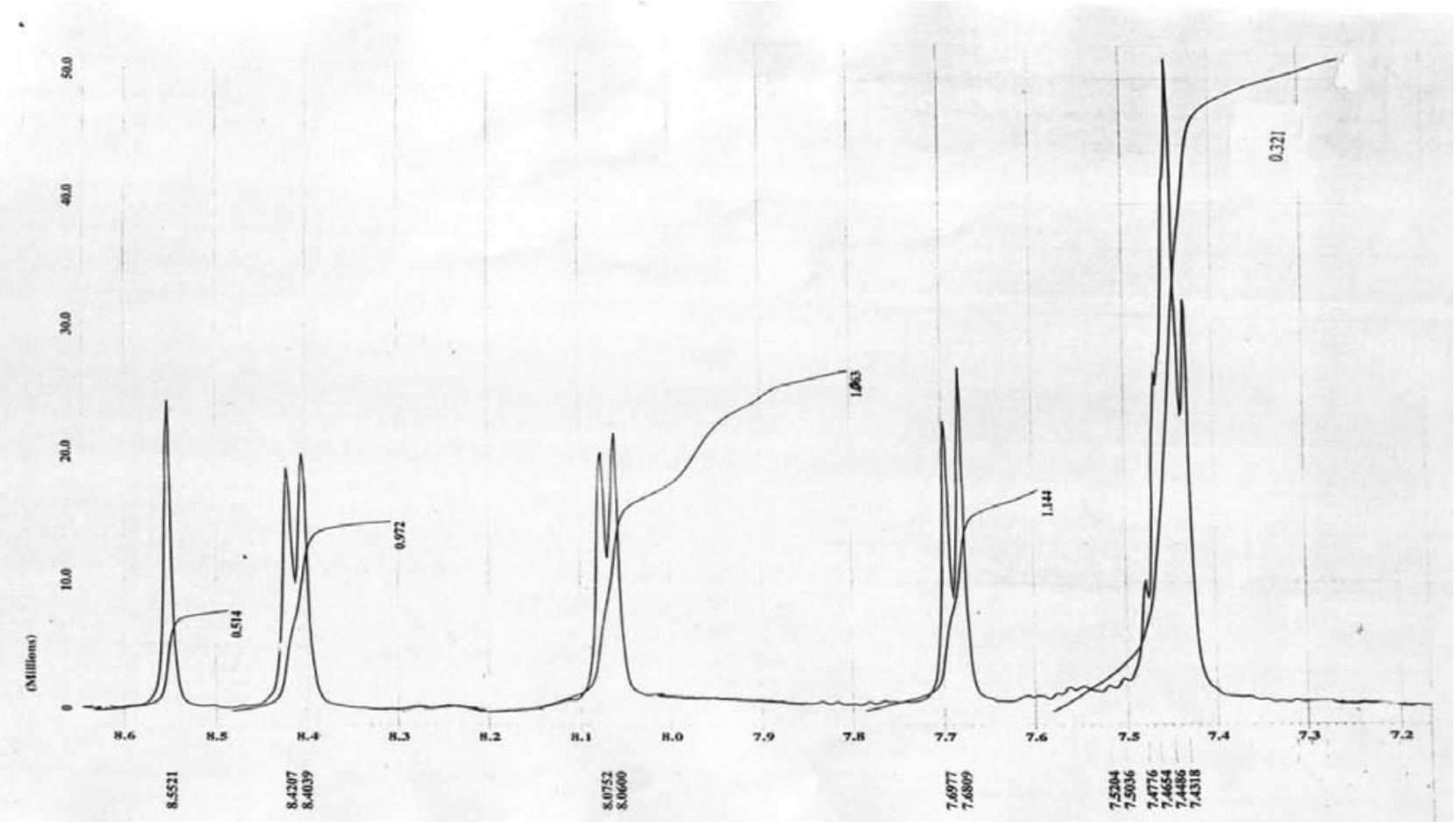

Figure S55. ${ }^{1} \mathrm{H}$ NMR spectrum of compound 26. 


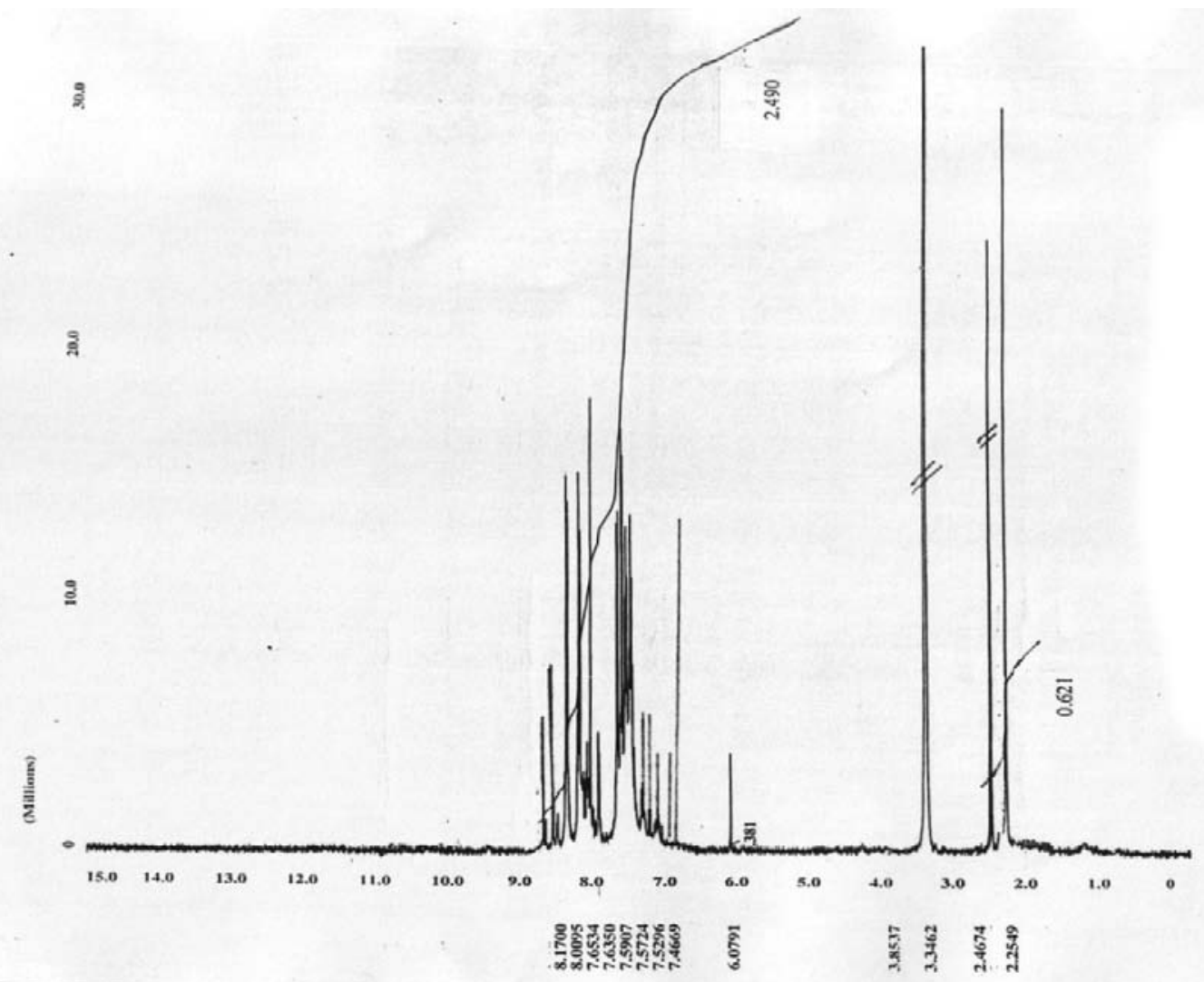

Figure S56. ${ }^{1} \mathrm{H}$ NMR spectrum of compound 33 .

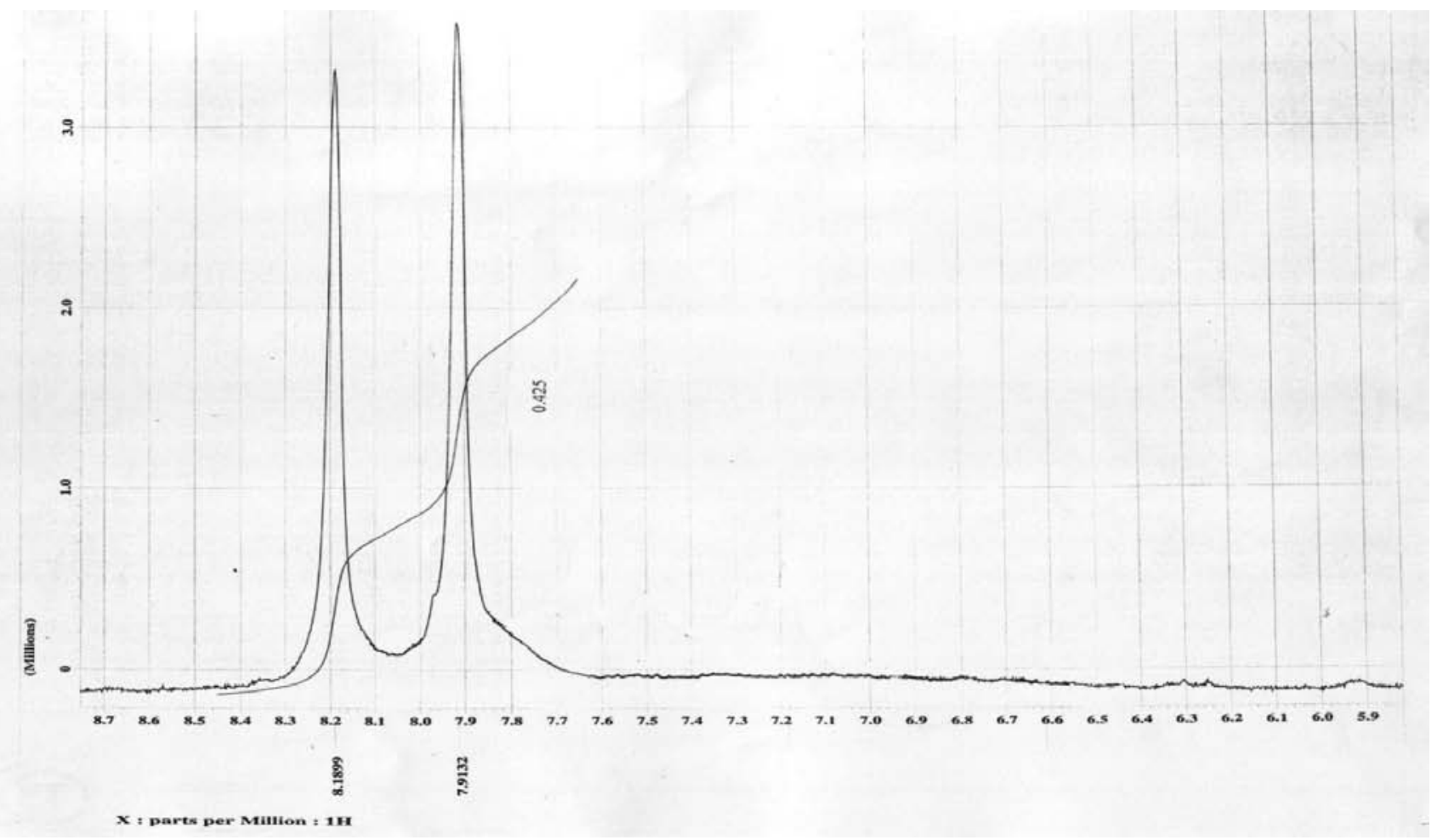

Figure S57. ${ }^{1} \mathrm{H}$ NMR spectrum of compound 33 . 


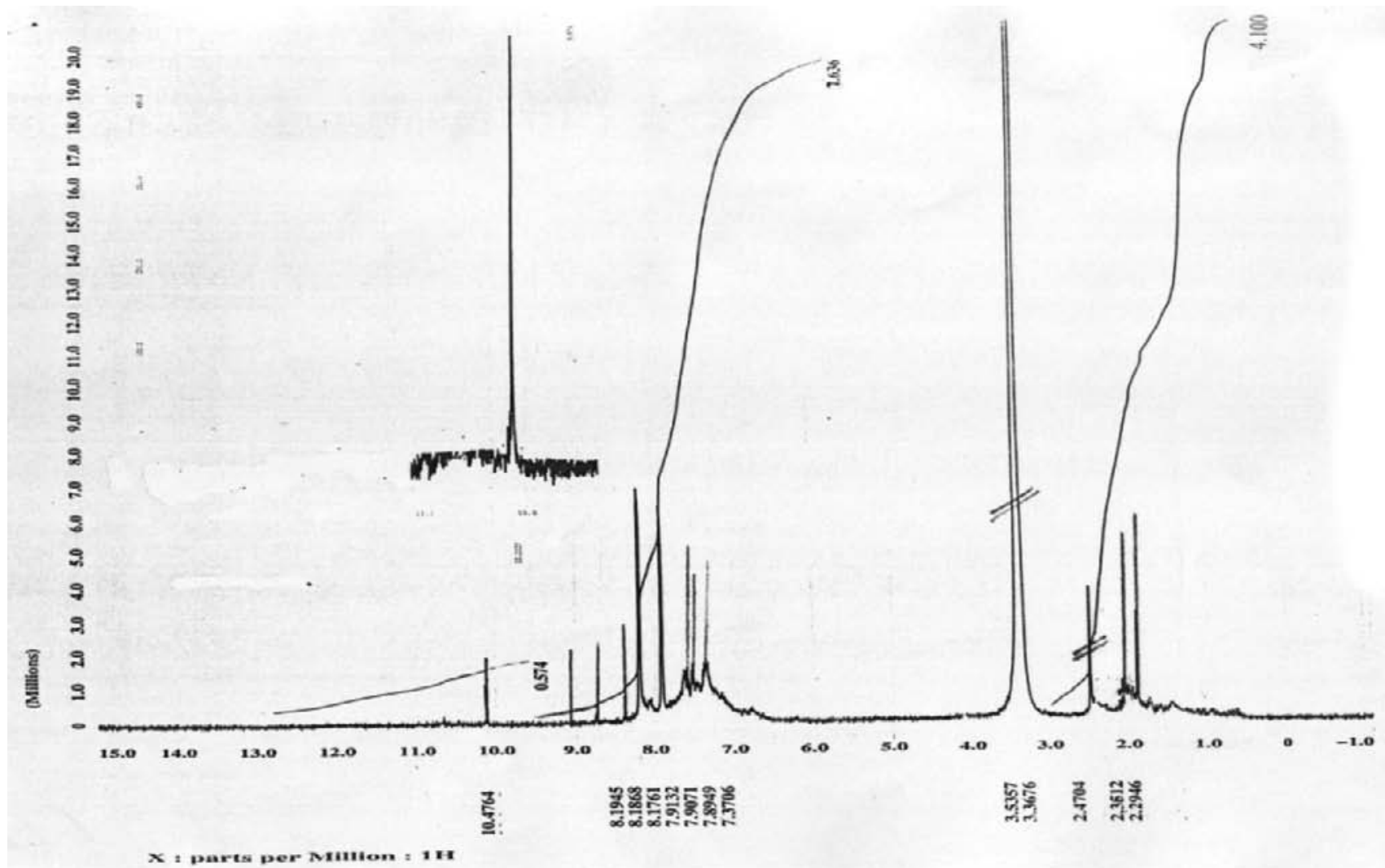

Figure S58. ${ }^{1} \mathrm{H}$ NMR spectrum of compound 36.

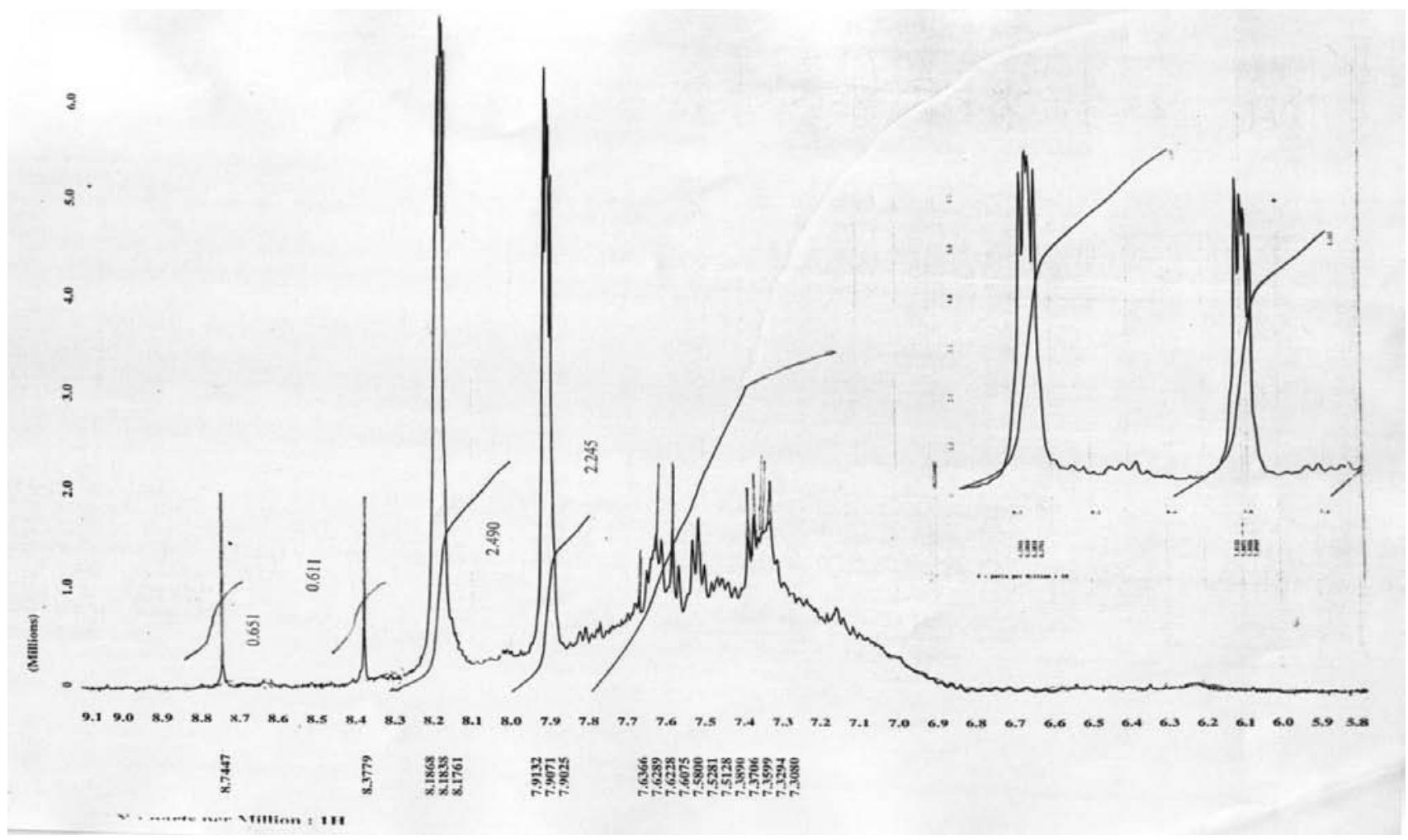

Figure S59. ${ }^{1} \mathrm{H}$ NMR spectrum of compound $\mathbf{3 6}$. 


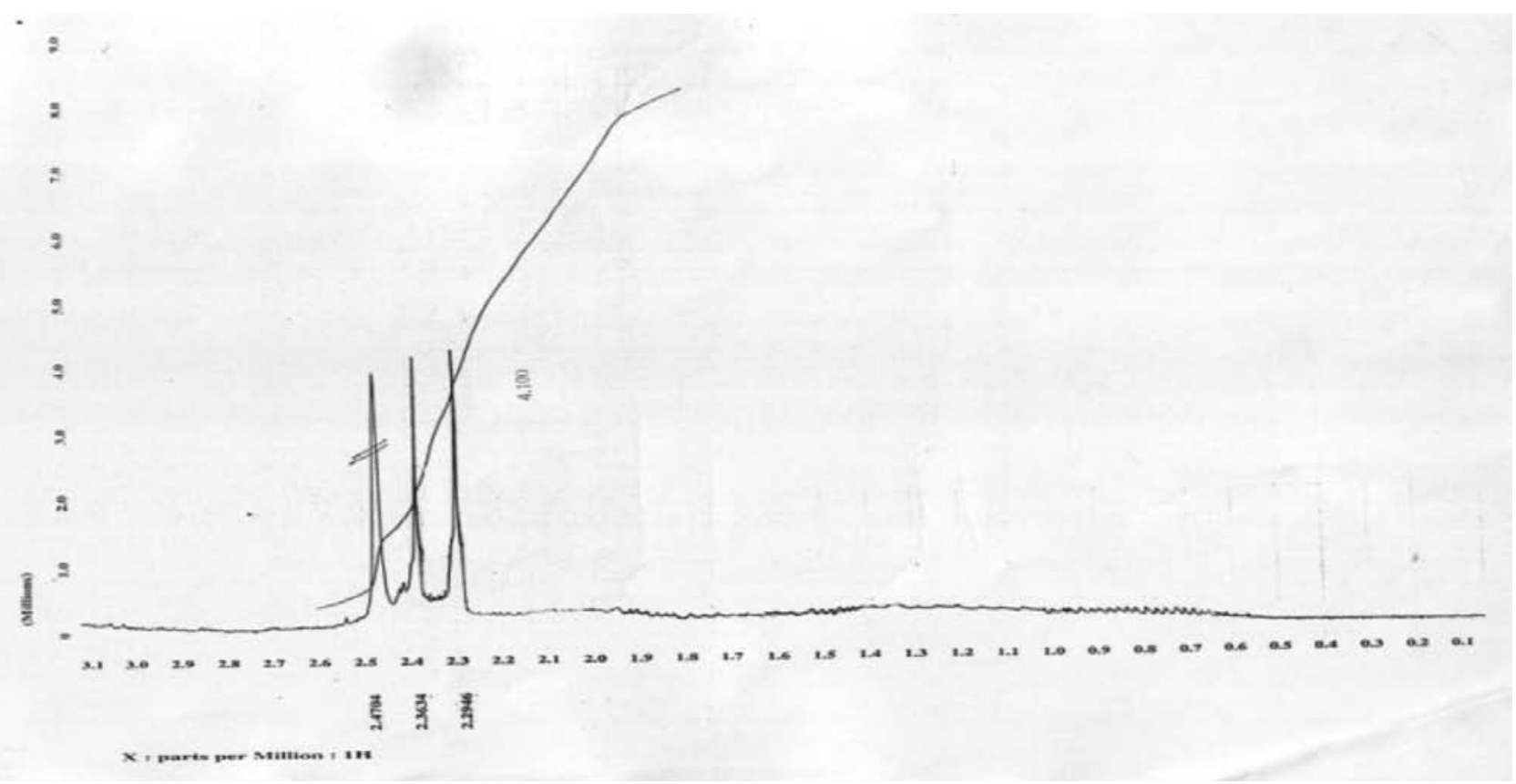

Figure S60. ${ }^{1} \mathrm{H}$ NMR spectrum of compound 36.

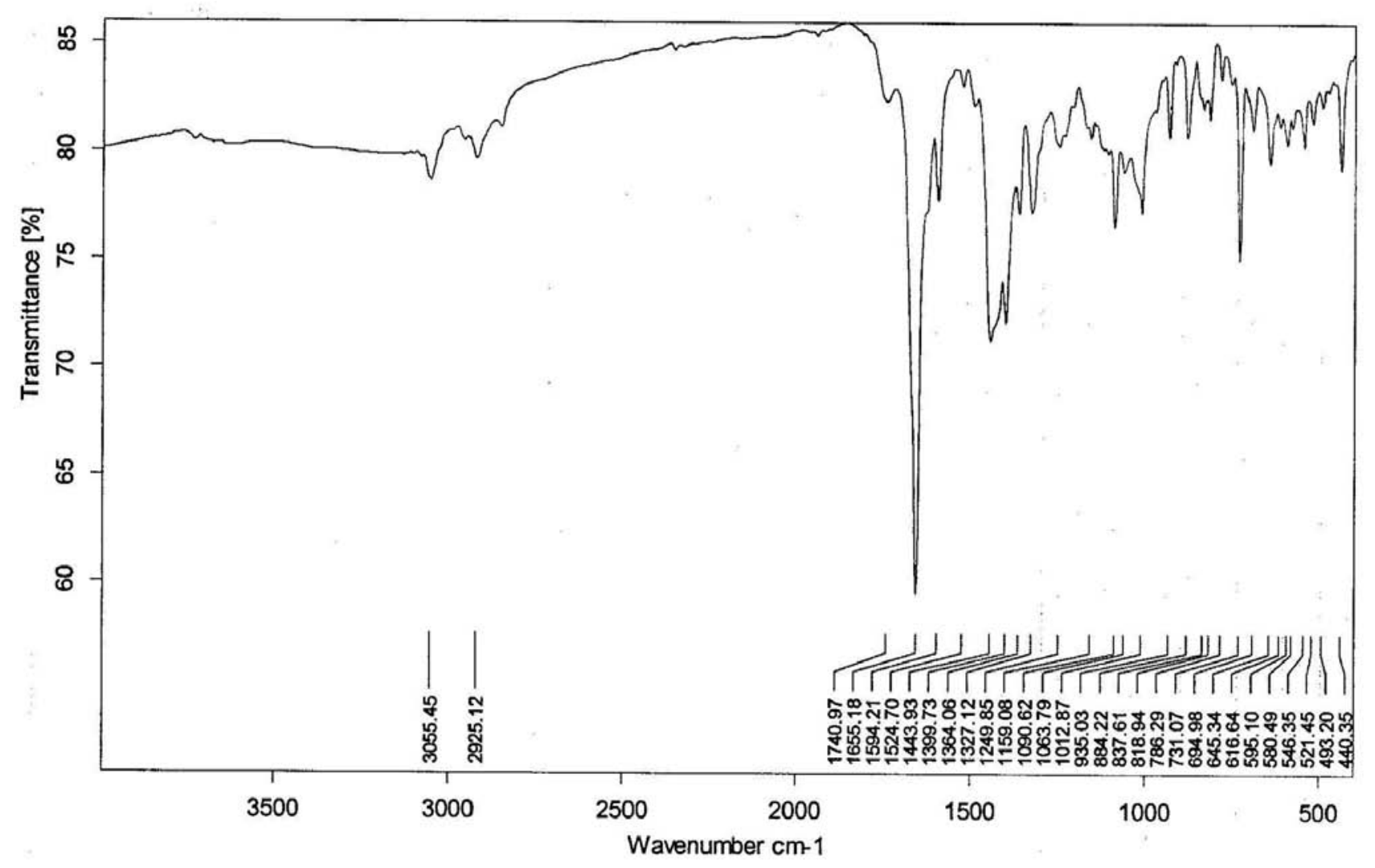

Figure S61. IR spectrum of compound 20. 


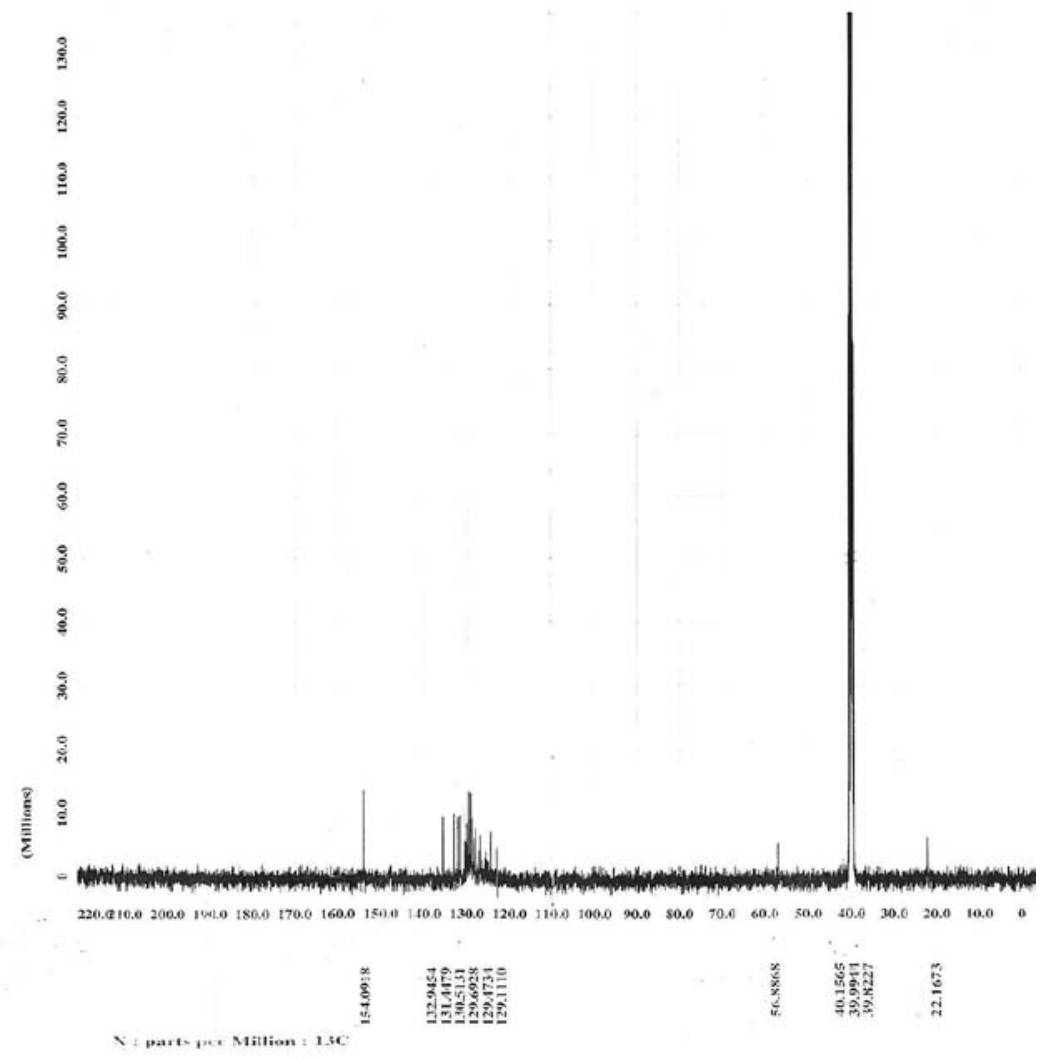

Figure S62. ${ }^{13} \mathrm{C}$ NMR spectrum of compound 20.

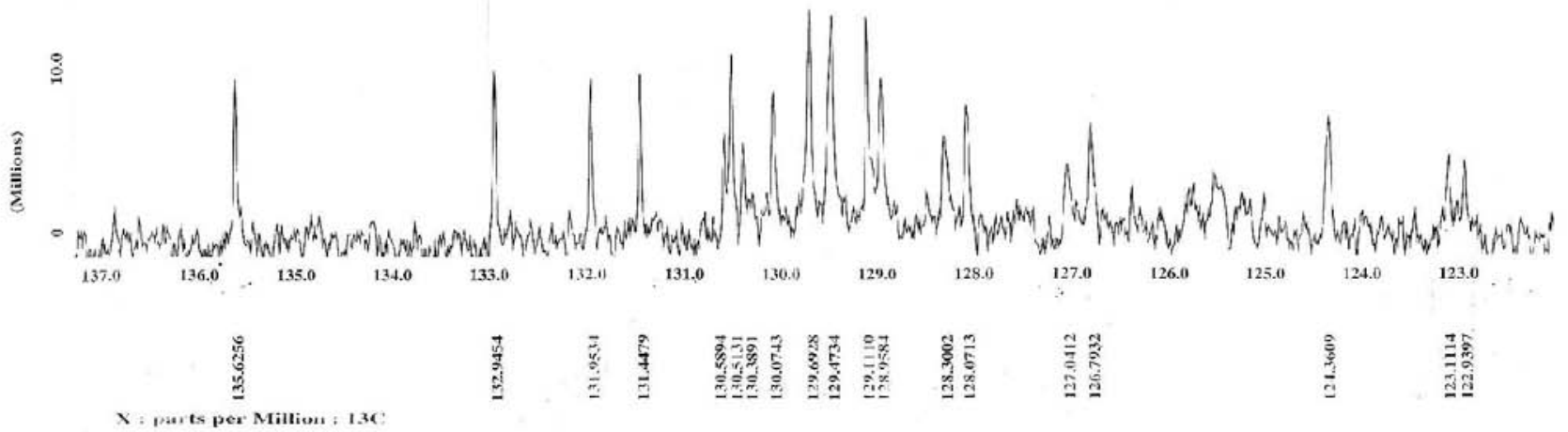

Figure S63. ${ }^{13} \mathrm{C}$ NMR spectrum of compound 20. 